JOURNAL OF THE AMERICAN MATHEMATICAL SOCIETY

Volume 18, Number 3, Pages 501-545

S 0894-0347(05)00482-0

Article electronically published on March 30, 2005

\title{
ON THE FARRELL-JONES CONJECTURE FOR HIGHER ALGEBRAIC $K$-THEORY
}

\author{
ARTHUR BARTELS AND HOLGER REICH
}

\section{INTRODUCTION}

Conjecturally the algebraic $K$-theory groups $K_{n}(\mathbb{Z} \Gamma), n \in \mathbb{Z}$, of the integral group ring $\mathbb{Z} \Gamma$ of every torsion free group $\Gamma$ can be expressed in terms of the group homology of $\Gamma$ and the algebraic $K$-theory of the integers. More precisely there is the following conjecture; compare [Hsi84, Section VI].

Conjecture 1.1. For a torsion free group $\Gamma$ the so-called assembly map [Lod76]

$$
A: H_{n}\left(B \Gamma ; \mathbb{K}^{-\infty}(\mathbb{Z})\right) \rightarrow K_{n}(\mathbb{Z} \Gamma)
$$

is an isomorphism for all $n \in \mathbb{Z}$.

Here $B \Gamma$ is the classifying space of the group $\Gamma$, and we denote by $\mathbb{K}^{-\infty}(R)$ the non-connective algebraic $K$-theory spectrum of the ring $R$. The homotopy groups of this spectrum are denoted $K_{n}(R)$ and coincide with Quillen's algebraic $K$-groups of $R$ Qui73 in positive dimensions and with the negative $K$-groups of Bass Bas68. in negative dimensions. The homotopy groups of the spectrum $X_{+} \wedge \mathbb{K}^{-\infty}(R)$ are denoted $H_{n}\left(X ; \mathbb{K}^{-\infty}(R)\right)$. They yield a generalized homology theory and, in particular, standard computational tools such as the Atiyah-Hirzebruch spectral sequence apply to the left-hand side of the assembly map above.

As a corollary of the main result of this paper we prove Conjecture 1.1 in the case where $\Gamma$ is the fundamental group of a closed Riemannian manifold with strictly negative sectional curvature. In fact our result is more general and applies to group rings $R \Gamma$, where $R$ is a completely arbitrary coefficient ring.

Note that if one replaces in Conjecture 1.1 the coefficient ring $\mathbb{Z}$ by an arbitrary coefficient ring $R$ the corresponding conjecture would be false already in the simplest non-trivial case: if $\Gamma=C$ is the infinite cyclic group the Bass-Heller-Swan formula [BHS64, Gra76, p. 236] for $K_{n}(R C)=K_{n}\left(R\left[t^{ \pm 1}\right]\right)$ yields that

$$
K_{n}(R C) \cong K_{n-1}(R) \oplus K_{n}(R) \oplus \mathrm{NK}_{n}(R) \oplus \mathrm{NK}_{n}(R)
$$

where $\mathrm{NK}_{n}(R)$ is defined as the cokernel of the split inclusion $K_{n}(R) \rightarrow K_{n}(R[t])$ and does not vanish in general. But since $S^{1}$ is a model for $B C$ one obtains on the left-hand side of the assembly map only

$$
H_{n}\left(B C ; \mathbb{K}^{-\infty}(R)\right) \cong K_{n}(R) \oplus K_{n-1}(R) .
$$

Received by the editors August 5, 2003.

2000 Mathematics Subject Classification. Primary 19D50; Secondary 53C12.

Key words and phrases. $K$-theory, group rings, controlled algebra.

(C)2005 American Mathematical Society Reverts to public domain 28 years from publication 
In some sense this is all that goes wrong. Combining our main result Theorem 1.4 with Proposition 1.8 in [BFJR04] we obtain the following generalization of the BassHeller-Swan formula.

Corollary 1.2. Let $\Gamma$ be a fundamental group of a closed Riemannian manifold with strictly negative sectional curvature. Then for every associative ring with unit $R$ we have

$$
K_{n}(R \Gamma) \cong H_{n}\left(B \Gamma ; \mathbb{K}^{-\infty}(R)\right) \oplus \bigoplus_{I} \mathrm{NK}_{n}(R) \oplus \mathrm{NK}_{n}(R)
$$

for all $n \in \mathbb{Z}$. Here the sum on the right is indexed over the set I of conjugacy classes of maximal cyclic subgroups of $\Gamma$.

Recall that a ring $R$ is called (right) regular if it is (right) Noetherian and every finitely generated (right) $R$-module admits a finite-dimensional projective resolution. Principal ideal domains are examples of regular rings. It is known Bas68, Chapter XII], Qui73, p. 122] that for a regular ring, $\mathrm{NK}_{n}(R)=0$ for all $n \in \mathbb{Z}$. Hence for regular coefficient rings the expression in Corollary 1.2 simplifies and proves the more general version of Conjecture 1.1 where the coefficient ring $\mathbb{Z}$ is replaced by an arbitrary regular coefficient ring $R$.

We proceed to describe the Farrell-Jones Conjecture concerning the algebraic $K$-theory of group rings [FJ93a, which is the correct conceptional framework for these kinds of results and which applies also to groups which contain torsion.

A set of subgroups of a given group $\Gamma$ is called a family of subgroups if it is closed under conjugation with elements from $\Gamma$ and closed under taking subgroups. We denote by

$$
\{1\}, \quad C y c, \quad V C y c \text { and } A l l
$$

the families which consist of the trivial subgroup, all cyclic subgroups, all virtually cyclic subgroups, respectively all subgroups of $\Gamma$. Recall that a group is called virtually cyclic if it contains a cyclic subgroup of finite index.

For every family $\mathcal{F}$ of subgroups of $\Gamma$ there exists a classifying space for the family $\mathcal{F}$ denoted $E \Gamma(\mathcal{F})$; compare [tD72, tD87 I.6], and [FJ93a, Appendix]. It is characterized by the universal property that for every $\Gamma$-CW-complex $X$ whose isotropy groups are all in the family $\mathcal{F}$ there exists an equivariant continuous map $X \rightarrow E \Gamma(\mathcal{F})$ which is unique up to equivariant homotopy. A $\Gamma$-CW-complex $E$ is a model for the classifying space $E \Gamma(\mathcal{F})$ if the fixpoint sets $E^{H}$ are contractible for $H \in \mathcal{F}$ and empty otherwise. Note that the one point space pt is a model for $E \Gamma(A l l)$ and that $E \Gamma(\{1\})$ is the universal covering of the classifying space $B \Gamma$.

In DL98 Davis and Lück construct a generalized equivariant homology theory for $\Gamma$-CW-complexes $X \mapsto H_{n}^{\Gamma}\left(X ; \mathbb{K}_{R}^{-\infty}\right)$ associated to a jazzed-up version of the non-connective algebraic $K$-theory spectrum functor. If one evaluates this $\Gamma$ homology theory on a homogeneous space $\Gamma / H$ one obtains $H_{n}^{\Gamma}\left(\Gamma / H ; \mathbb{K}_{R}^{-\infty}\right) \cong$ $K_{n}(R H)$. Using this language the Farrell-Jones Conjecture for the algebraic $K$ theory of group rings can be formulated as follows.

Conjecture 1.3 (The Farrell-Jones Conjecture for $K_{n}(R \Gamma)$ ). Let $\Gamma$ be a group and let $R$ be an associative ring with unit. Then the map

$$
A_{V C y c}: H_{n}^{\Gamma}\left(E \Gamma(V C y c) ; \mathbb{K}_{R}^{-\infty}\right) \rightarrow H_{n}^{\Gamma}\left(\mathrm{pt} ; \mathbb{K}_{R}^{-\infty}\right) \cong K_{n}(R \Gamma)
$$

which is induced by the projection $E \Gamma(V C y c) \rightarrow$ pt is an isomorphism for all $n \in \mathbb{Z}$. 
This conjecture was formulated in [FJ93a for $R=\mathbb{Z}$ and stated in this more general form in BFJR04]. Above we used the language developed by Davis and Lück in DL98 to formulate the conjecture. The identification of this formulation with the original formulation in [FJ93a] which used Qui82 is carried out in [HP04]. For more information on this and related conjectures the reader should consult LR04.

Our main result proves Conjecture 1.3 for the class of groups that was already mentioned above.

Theorem 1.4. Let $R$ be an associative ring with unit. Let $\Gamma$ be the fundamental group of a closed Riemannian manifold with strictly negative sectional curvature. Then for all $n \in \mathbb{Z}$ the assembly map

$$
A_{C y c}: H_{n}^{\Gamma}\left(E \Gamma(C y c) ; \mathbb{K}_{R}^{-\infty}\right) \rightarrow K_{n}(R \Gamma)
$$

induced by the projection $E \Gamma(C y c) \rightarrow \mathrm{pt}$ is an isomorphism.

Note that the groups considered in this theorem are torsion free and that for a torsion free group the family $V C y c$ of all virtually cyclic subgroups reduces to the family $C y c$ of all cyclic subgroups. The result extends the results of [BFJR04, where surjectivity in low dimensions and injectivity were proven. Results which are strongly related to the result above concerning the low-dimensional $K$-theory of the integral group ring, pseudoisotopy spectra and the structure set in surgery theory were proven by Farrell and Jones in [FJ86], FJ87, FJ89] and FJ91. Apart from Wal78 the result above seems to be the first integral result of this type which applies to the higher algebraic $K$-theory of group rings.

From the fact that we do not make any assumptions on the coefficient ring $R$ one can derive a corresponding isomorphism statement for an assembly map for NK-groups.

Corollary 1.5. Let $R$ be an associative ring with unit. Let $\Gamma$ be the fundamental group of a closed Riemannian manifold with strictly negative sectional curvature. Then for all $n \in \mathbb{Z}$ the assembly map for NK-groups

$$
A_{C y c}: H_{n}^{\Gamma}\left(E \Gamma(C y c) ; \mathbb{N K}_{R}^{-\infty}\right) \rightarrow H_{n}^{\Gamma}\left(\mathrm{pt} ; \mathbb{N K}_{R}^{-\infty}\right) \cong \mathrm{NK}_{n}(R \Gamma)
$$

is an isomorphism.

Proof. Since there is a splitting $\mathbb{K}_{R[t]}^{-\infty} \simeq \mathbb{K}_{R}^{-\infty} \vee \mathbb{N}_{R}^{-\infty}$ the isomorphism result for two of the assembly maps associated to $\mathbb{K}_{R}^{-\infty}, \mathbb{K}_{R[t]}^{-\infty}$, respectively $\mathbb{N}_{R}^{-\infty}$ implies the result for the third; compare [BFJR04, Proposition 7.4].

In particular one can conclude that the vanishing of $\mathrm{NK}_{n}(R)$ and $\mathrm{NK}_{n}(R[t])$ for $n \leq N$ implies the vanishing of $\mathrm{NK}_{n}(R \Gamma)$ for $n \leq N$. Note that even if $R$ is regular it is not at all clear if $R \Gamma$ is regular. If one uses that $R\left[\Gamma_{1} \times \Gamma_{2}\right]=R\left[\Gamma_{1}\right]\left[\Gamma_{2}\right]$ and iterates one obtains the following corollary.

Corollary 1.6. Suppose $\Gamma=\Gamma_{1} \times \Gamma_{2} \times \cdots \times \Gamma_{k}$, where each $\Gamma_{i}$ is the fundamental group of a closed Riemannian manifold with strictly negative sectional curvature. If $R$ is a regular ring, then the assembly map

$$
A: H_{n}\left(B \Gamma ; \mathbb{K}^{-\infty}(R)\right) \rightarrow K_{n}(R \Gamma)
$$

is an isomorphism for all $n \in \mathbb{Z}$. 
The proof of Theorem 1.4 relies on the fact (see Subsection 2.2 and [BFJR04]) that the assembly map $A_{V C y c}$ can be described as a "forget-control map" in the sense of controlled topology; compare Qui82, [PW89. In order to prove a surjectivity result we hence have to "gain control". More precisely the negatively curved manifold $M$ whose fundamental group we want to treat can be used in order to construct a geometric model for the map $E \Gamma \rightarrow E \Gamma(C y c)$ given by the universal property. We will consider suitable additive categories of $R$-modules and morphisms over $E \Gamma \times[1, \infty)$ where the morphisms satisfy control conditions. The assembly map is obtained by applying $K$-theory to an inclusion of additive categories, where the larger category differs from the smaller one by a relaxed control condition on the morphisms.

The geometric program for gaining control stems from [FJ86] and consists mainly of three steps:

(I) Construct a transfer from the manifold $M$ to the sphere bundle $S M$ in such a way that transferred morphisms are in asymptotic position; i.e., they are in a good starting position for the geodesic flow. In order to make sure that transferring up and projecting down again does not change the $K$ theory class, one has to work with a slightly modified bundle in place of the sphere bundle.

(II) Consider the foliation on the sphere bundle $S M$ given by the flow lines of the geodesic flow. Use the geodesic flow on $S M$ in order to achieve "foliated control".

(III) Prove a "Foliated Control Theorem" in order to improve from "foliated control" to "ordinary control". At least do so away from the "short" closed geodesics.

Note that the closed geodesics that appear in Step (III) are in bijection with conjugacy classes of cyclic subgroups. Hence the family Cyc which appears in Theorem 1.4 shows up quite naturally in the proof.

We refer to Section 3 for a more detailed outline of the proof. In the following we only discuss why new techniques were necessary in order to treat higher algebraic $K$-theory along the lines of the program above.

One main difficulty was to construct a suitable transfer as required in Step (I) of the program. Looking at the analogous situations for $h$-cobordisms or $A$-theory, where a transfer is given by pull-back, it is in principle clear what the algebraic analogue in our situation should be. However the obvious naive approaches are not "functorial enough" to induce a map in higher $K$-theory. Hence one needs to come up with a suitably refined transfer which takes care of the functoriality problems (e.g. work with singular chain complexes) but at the same time does not destroy the control requirements. In order to treat the question whether transferring up and projecting down yields the identity on $K$-theory we prove in Proposition 5.9 a formula for the kind of transfers we construct. Transferring up and projecting down yields multiplication by a certain element in the Swan group. The Swan group element depends on the homology groups of the fiber considered as modules over the fundamental group of the base.

Another main achievement in this paper is the Foliated Control Theorem 6.17 for higher algebraic $K$-theory. Earlier foliated control theorems (see for example Theorem 1.6 in [FJ86] or Theorem 1.1 in [BFJR03]) were formulated for individual $K$-theory elements. It is however difficult to explicitly describe elements in higher 
$K$-theory groups. Hence we had to find a way to formulate and prove a foliated control theorem in a more "functorial" fashion. We would like to emphasize that the Foliated Control Theorem 6.17]does not rely on a squeezing result or any kind of torus trick. Essentially only the existence of the long exact sequence associated to a Karoubi filtration [CP97] and Eilenberg swindles are used as the abstract building blocks for our argument. Of course on the geometric side the existence of long-andthin cell structures proven in FJ86 is crucial. In particular our technique should prove analogous foliated control theorems in the context of algebraic $L$-theory or topological $K$-theory of $C^{*}$-algebras since the corresponding tools are available in those set-ups; compare [HPR97.

The reader who is familiar with the work of Farrell-Jones (in particular [FJ91] and [FJ93b]) may wonder why we cannot weaken the assumption in Theorem 1.4 from strictly negative curvature to non-positive curvature. The reason is that the focal transfer which is used in [FJ91] and FJ93b], in contrast to the asymptotic transfer used in this paper, is definitely not functorial, and it is hence even more difficult to describe a corresponding transfer for higher algebraic $K$-theory.

It also remains open whether the program can be adapted to prove cases of the Baum-Connes Conjecture BCH94 or to treat algebraic L-theory with arbitrary coefficients. In both cases a crucial question is whether a suitable transfer can be constructed. (A geometric version of an $L$-theory transfer is one of the many ingredients in [FJ89.)

The paper is organized as follows.

1 Introduction

2 Preliminaries

3 Outline of the proof

4. Gaining control via the geodesic flow

5. The transfer

6] A foliated control theorem for higher $K$-theory

8 Appendix

References

\section{Preliminaries}

2.1. Conventions and notation. In this section we briefly introduce some notation that is used throughout the proof. For more details the reader should consult Section 2 in [BFJR04].

2.1.1. The functor $\mathbb{K}^{-\infty}$. We will denote by $\mathbb{K}^{-\infty}$ the functor which associates to an additive category its non-connective $K$-theory spectrum; see [PW85] or [CP97]. We assume that the reader is familiar with the standard properties of this functor; compare [BFJR04, Subsection 2.1]. A statement about exact functors between additive categories being true "after applying $K$-theory" or "on the level of $K$ theory" will always mean after applying $\mathbb{K}^{-\infty}$.

2.1.2. Modules and morphisms over a space. As explained below in Subsection 2.2 the assembly map will be described as a "forget-control map" between suitable additive categories of (geometric) modules. An $R$-module $M$ over the space $X$ is a family $\left(M_{x}\right)_{x \in X}$ of finitely generated free $R$-modules $M_{x}$ indexed over points of $X$, which is locally finite in the sense that $\bigoplus_{x \in K} M_{x}$ is finitely generated for every compact subset $K \subset X$. A morphism $\phi: M \rightarrow N$ is an $R$-linear map 
$\phi: \bigoplus_{x \in X} M_{x} \rightarrow \bigoplus_{y \in X} N_{y}$. Such a map can of course be decomposed and written as a matrix $\phi=\left(\phi_{y, x}\right)$ indexed over $X \times X$. The additive category of all such modules and morphisms will be denoted $\mathcal{C}(X)$ or $\mathcal{C}(X ; R)$ and is equivalent to the category of finitely generated free $R$-modules if $X$ is a compact space.

2.1.3. Support conditions. We are however only interested in subcategories of modules and morphisms satisfying certain support conditions. The support of a module $M$ (or a morphism $\phi$ ) is defined as $\operatorname{supp} M=\left\{x \in X \mid M_{x} \neq 0\right\} \subset X$ (respectively $\left.\operatorname{supp} \phi=\left\{(x, y) \mid \phi_{y, x} \neq 0\right\} \subset X \times X\right)$. For a morphism control condition $\mathcal{E}$ (a set of subsets of $X \times X$ ) and an object support condition $\mathcal{F}$ (a set of subsets of $X$ ) we denote by

$$
\mathcal{C}(X, \mathcal{E}, \mathcal{F})
$$

the subcategory of $\mathcal{C}(X)$ consisting of modules $M$, for which there exists an $F \in \mathcal{F}$ such that $\operatorname{supp} M \subset F$, and morphisms $\phi$ between such modules, for which there exists an $E \in \mathcal{E}$ with $\operatorname{supp} \phi \subset E$. We will often refer to such morphisms as $\mathcal{E}$ controlled morphisms. The conditions one needs to impose on $\mathcal{E}$ and $\mathcal{F}$ in order to assure that this yields in fact an additive category are spelled out in Subsection 2.3 of BFJR04. A basic example of a morphism control condition on a metric space $(X, d)$ is $\mathcal{E}_{d}$, consisting of all $E \subset X \times X$ for which there is $\alpha>0$ such that $(x, y) \in E$ implies $d(x, y)<\alpha$. Measuring control via a map $p: X \rightarrow Y$ is formalized by pulling back $\mathcal{E}$ (living on $Y$ ), i.e. forming $p^{-1} \mathcal{E}=\left\{(p \times p)^{-1}(E) \mid E \in \mathcal{E}\right\}$. In the case where $p$ is the inclusion of a subspace we usually omit $p$ and write $\mathcal{E}$ instead of $p^{-1} \mathcal{E}$. Similar notational conventions apply to the $\mathcal{F}$ 's.

2.1.4. Equivariant versions. We usually deal with equivariant versions where $X$ is assumed to be a free $\Gamma$-space and modules and morphisms are required to be invariant under the $\Gamma$-action. The corresponding category is denoted

$$
\mathcal{C}^{\Gamma}(X, \mathcal{E}, \mathcal{F}) .
$$

Under suitable conditions about the $\mathcal{E}$ 's and $\mathcal{F}$ 's a $\Gamma$-equivariant map $f: X \rightarrow Y$ induces a functor on such categories which sends $M$ (a module over $X$ ) to $f_{*} M$ (a module over $Y$ ) given by $f_{*} M_{y}=\bigoplus_{x \in f^{-1}(\{y\})} M_{x}$.

2.1.5. Thickenings. If $E$ is a neighborhood of the diagonal in $X \times X$ and $A$ a subset of $X$, then we define the $E$-thickening $A^{E}$ of $A$ in $X$ to be the set of all points $x \in X$ for which there exists a point $a \in A$ such that $(a, x)$ belongs to $E$. In the case where $E$ is determined by a constant $\delta$ via a metric or by a pair $(\alpha, \delta)$ using the "foliated distance" (compare Subsection 4.2) we write $A^{\delta}$, respectively $A^{\alpha, \delta}$, for the corresponding thickenings.

2.1.6. Germs. We will often use Karoubi quotients of the categories $\mathcal{C}^{\Gamma}(X, \mathcal{E}, \mathcal{F})$ introduced above: let $\mathcal{F}_{0}$ be another object support condition which is $\mathcal{E}$-thickening closed, i.e. for every $F \in \mathcal{F}_{0}$ and $E \in \mathcal{E}$ there exists an $F^{\prime} \in \mathcal{F}_{0}$ such that $F^{E} \subset F^{\prime}$. Then $\mathcal{C}^{\Gamma}(X, \mathcal{E}, \mathcal{F})^{>\mathcal{F}_{0}}$ is defined as the additive category which has the same objects as $\mathcal{C}^{\Gamma}(X, \mathcal{E}, \mathcal{F})$, but where morphisms are identified whenever their difference factors over a module with support in $F_{0} \in \mathcal{F}_{0}$. We think of this construction as taking germs away from $\mathcal{F}_{0}$. If our space is $X \times[1, \infty)$ and $\mathcal{F}_{0}=\{X \times[1, t] \mid t \in$ $[1, \infty)\}$, then we write $\mathcal{C}^{\infty}$ rather than $\mathcal{C}^{>\mathcal{F}_{0}}$; see Example 8.8. Some further formal properties of these constructions are discussed in Appendix 8.4. 


\subsection{Assembly as a "forget control-map".}

2.2.1. Resolutions. A $\Gamma$-space is called $\Gamma$-compact if it is the $\Gamma$-orbit of some compact subspace. A resolution of the $\Gamma$-space $X$ is a $\Gamma$-map $p: \bar{X} \rightarrow X$ of $\Gamma$-CWcomplexes, where $\bar{X}$ is a free $\Gamma$-space and every $\Gamma$-compact set in $X$ is the image under $p$ of some $\Gamma$-compact set in $\bar{X}$. For every space the projection $X \times \Gamma \rightarrow X$ is a functorial resolution called the standard resolution.

2.2.2. The functor $\mathcal{D}^{\Gamma}$. In [BFJR04, Definition 2.7] we defined for a not necessarily free $\Gamma$-CW complex $X$ the notion of $\Gamma$-equivariant continuous control. This is a morphism support condition denoted $\mathcal{E}_{\Gamma c c}(X)$ on the space $X \times[1, \infty)$. The set of all $\Gamma$-compact subsets of $\bar{X}$ is denoted $\mathcal{F}_{\Gamma c}(\bar{X})$. The object support condition $p_{\bar{X}}^{-1} \mathcal{F}_{\Gamma c}(\bar{X})$, where $p_{\bar{X}}: \bar{X} \times[1, \infty) \rightarrow \bar{X}$ denotes the projection, is our standard object support condition on $\bar{X} \times[1, \infty)$. It is shown in [BFJR04, Sections 3 and 5] that up to equivalence

$$
\mathcal{D}^{\Gamma}(\bar{X} ; p)=\mathcal{C}^{\Gamma}\left(\bar{X} \times[1, \infty) ;(p \times \mathrm{id})^{-1} \mathcal{E}_{\Gamma c c}(X), p_{\bar{X}}^{-1} \mathcal{F}_{\Gamma c}(\bar{X})\right)
$$

does not depend upon the chosen resolution (hence one can always use $X \times \Gamma \rightarrow X$ as a resolution; in this case we denote the category by $\left.\mathcal{D}^{\Gamma}(X)\right)$ and that $X \mapsto$ $\mathbb{K}^{-\infty} \mathcal{D}^{\Gamma}(X)$ yields an equivariant homology theory on the level of homotopy groups.

\subsubsection{Assembly. The map induced by}

$$
\mathcal{D}^{\Gamma}(E \Gamma(V C y c)) \rightarrow \mathcal{D}^{\Gamma}(\mathrm{pt})
$$

is, on the level of $K$-theory up to an index shift, a model for the generalized assembly map that was discussed above. Compare [BFJR04, Corollary 6.3].

\section{Outline of the PROOF}

The injectivity part of Theorem [1.4 is proven in [BFJR04]. We will prove surjectivity. Our first observation is that it suffices to prove surjectivity of the map which is induced on the level of $K$-theory by $\mathcal{D}^{\Gamma}(X(\infty)) \rightarrow \mathcal{D}^{\Gamma}(\mathrm{pt})$, where $X(\infty)$ is any $\Gamma-C W$ complex all of whose non-trivial isotropy groups are infinite cyclic. In fact by the universal property of $E \Gamma(C y c)$ such a map always factorizes over $\mathcal{D}^{\Gamma}(E \Gamma(C y c))$. It follows from [BFJR04, Proposition 3.5] that instead of considering the map of standard resolutions (compare 2.2.1) we can equally well work with any map of resolutions which covers $X(\infty) \rightarrow$ pt. We proceed to construct a space $X(\infty)$ and such a map of resolutions.

Let $\tilde{M}$ be the universal covering of a closed Riemannian manifold with strictly negative sectional curvature. The hyperbolic enlargement of $\tilde{M}$ is the warped product (compare [BO69])

$$
\mathbb{H} \tilde{M}=\mathbb{R} \times \cosh (t) \tilde{M} .
$$

It is the differentiable manifold $\mathbb{R} \times \tilde{M}$ equipped with the Riemannian metric determined by $d g_{\mathbb{H} \tilde{M}}^{2}=d t^{2}+\cosh (t)^{2} d g_{\tilde{M}}^{2}$. We refer to the $\mathbb{R}$-factor as the hyperbolic enlargement direction or briefly the $\mathbb{H}$-direction. Let $S \mathbb{H} \tilde{M}$ denote the unit-sphere subbundle of the tangent bundle of $\mathbb{H} \tilde{M}$. For a subset $A \subset \mathbb{R}$ we denote by $S \mathbb{H}_{A} \tilde{M}$ the restriction of this bundle to the subspace $\mathbb{H}_{A} \tilde{M} \subset \mathbb{H} \tilde{M}$ which is defined as $A \times \tilde{M} \subset \mathbb{R} \times \tilde{M}=\mathbb{H} \tilde{M}$. Throughout the paper we also fix the notation

$$
\mathbb{B}=[0, \infty) \text { and } \mathbb{T}=[1, \infty)
$$


The space $S \mathbb{H} \tilde{M} \times \mathbb{B} \times \mathbb{T}$ will be important in our context and we will generically use $h, \beta$ and $t$ to denote its $\mathbb{H}$-, $\mathbb{B}$ - and $\mathbb{T}$-coordinates.

In Section [7 we factorize the natural projection $S \mathbb{H} \tilde{M} \rightarrow \tilde{M}$ via a map called $p_{X}$ over a certain $\Gamma$-compact free $\Gamma$-space $X$; i.e., we have a commutative diagram

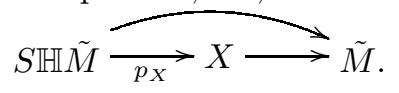

Roughly speaking $X$ is obtained from $S \mathbb{H} \tilde{M}$ by collapsing $S \mathbb{H}_{(-\infty,-1]} \tilde{M}$ along the $\mathbb{H}$-direction to $S \mathbb{H}_{\{-1\}} \tilde{M}$ and similarly $S \mathbb{H}_{[1, \infty)} \tilde{M}$ to $S \mathbb{H}_{\{1\}} \tilde{M}$. Note in particular that $S \tilde{M} \subset S \mathbb{H}_{\{0\}} \tilde{M}$ sits naturally as a subspace in $X$. For details about the map $p_{X}$ see Subsection 7.1 (resp. BFJR04, 14.5]). In Subsection 7.1 we also construct a quotient map

$$
p: X \times \mathbb{B} \rightarrow X(\infty) .
$$

Here $X(\infty)$ is a $\Gamma$-space all of whose isotropy groups are cyclic. It is obtained as the infinite mapping telescope of a sequence of maps which collapse more and more lines in $S \tilde{M} \subset X$. Here the $\mathbb{B}$-direction is the telescope direction and the lines correspond to preimages of closed flow lines of the geodesic flow under the covering projection $S \tilde{M} \rightarrow S M$. (Although $X(\infty)$ is not a model for $E \Gamma(C y c)$ it is fairly close to being one. With more effort one could probably work with an actual model in its place.) The map of resolutions alluded to above is now

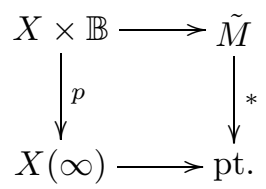

This map of resolutions induces the bottom map in the following diagram of additive categories:

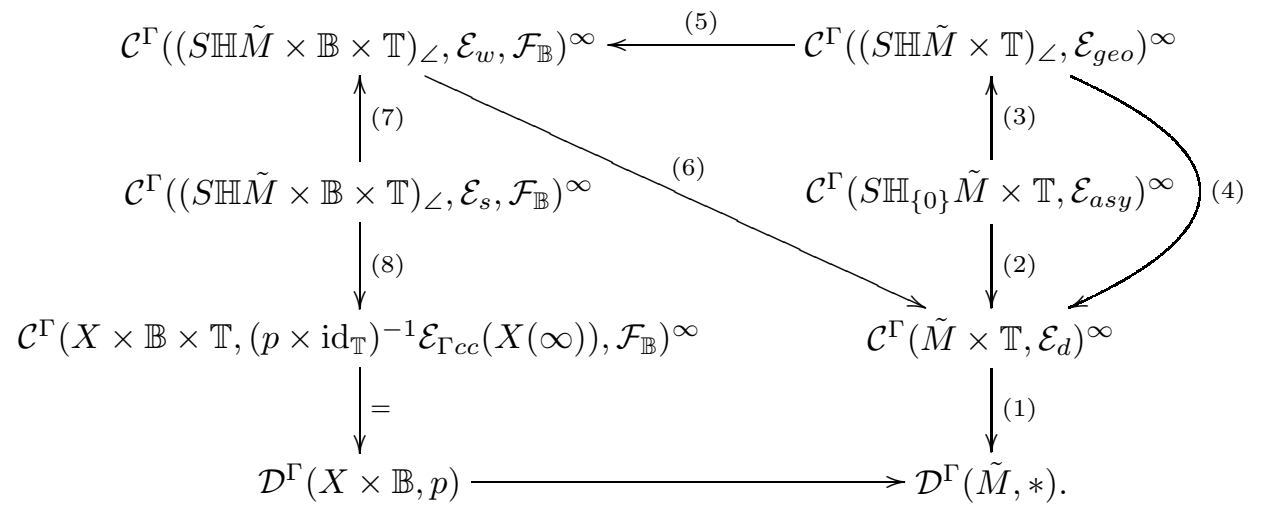

We first explain two spaces appearing in the diagram that have not yet been defined. The space $(S \mathbb{H} \tilde{M} \times \mathbb{T}) \angle$ is the subspace of $S \mathbb{H} \tilde{M} \times \mathbb{T}$ consisting of all points $(v, t)$ where the absolute value of the $\mathbb{H}$-coordinate satisfies $|h(v)| \leq t$. Similarly the subspace $(S \mathbb{H} \tilde{M} \times \mathbb{B} \times \mathbb{T}) \angle$ consists of all $(v, \beta, t)$ with $|h(v)| \leq t+\mu_{n} \beta$. The constant here is $\mu_{n}=10^{n+3}$, where $n$ is the dimension of $S \mathbb{H} \tilde{M}$. (The subscript $\angle$ is supposed to remind the reader of the shape of the region it describes.) We will mostly be interested in these subspaces, but all maps and all object and morphism 
support conditions can and will be defined on the entire space. The corresponding restrictions to the $\angle$-subspaces will not appear in the notation.

All maps in the diagram except (3) and (8) are induced by the obvious projections, inclusions or identity maps of the underlying spaces. The map (3) is essentially given by the geodesic flow and is discussed in detail in Section 4 The map of spaces underlying (8) is induced by $p_{X}: S \mathbb{H} \tilde{M} \rightarrow X$.

The essential information in the diagram is however contained in the different object and morphism support conditions which will be explained in detail below. Going clockwise around the diagram from the lower left-hand corner to the lower right-hand corner should be thought of as forgetting more and more control. Our task is to step-by-step gain control going counterclockwise. (The existence of the wrong way maps (5) and (8) says that in between we gain more control than we actually need.)

We have the following statements about the diagram above:

(i) It will be clear from the construction that the diagram without the map (3) commutes.

(ii) The triangle consisting of the bended arrow (4) and the maps (3) and (2) induces a triangle in $K$-theory which commutes up to homotopy. This will be proven in Corollary 5.10 .

(iii) The map (2) induces a split surjection in $K$-theory and hence by (ii) also the maps (4) and (6). This is an immediate consequence of Proposition 5.1 (compare the discussion before that Proposition).

(iv) The map (7) induces an equivalence in $K$-theory by the Foliated Control Theorem 6.17

(v) The map (1) induces an equivalence in $K$-theory by the easy Lemma 3.1 .

These statements imply that the bottom map induces a split surjection in $K$-theory, and hence our main Theorem 1.4 follows.

We will now describe the diagram in more detail and explain some aspects of statements (ii), (iii) and (iv) We proceed counterclockwise.

(1) Equip $\mathbb{T}$ with the standard metric. Let $d$ denote any product metric (e.g. the max-metric) on $\tilde{M} \times \mathbb{T}$ and let $\mathcal{E}_{d}$ be the corresponding morphism support condition. Observe that the continuous-control condition $*^{-1} \mathcal{E}_{\Gamma c c}$ is a weaker condition. The resulting forget-control map (1) is shown to induce an equivalence in Lemma 3.1 below.

(2) The base space of the bundle $S \mathbb{H}_{\{0\}} \tilde{M}$ is $\tilde{M}$. The map (2) is induced by the bundle projection. The space $S \mathbb{H} \tilde{M}$ comes equipped with two different foliations, the asymptotic foliation $F_{a s y}$ and the geodesic foliation $F_{g e o}$. These are explained in Section 4. In that section we will also define the notion of foliated control with a prescribed decay speed depending on a foliation $F$ and a set of decay speed functions $\mathcal{S}$. The important point about the map (2) is that in the source we have foliated control with a certain carefully chosen decay speed (denoted $\mathcal{S}_{a s y}$ ) with respect to the asymptotic foliation $F_{a s y}$. This kind of control will be denoted $\mathcal{E}_{\text {asy }}$. Section 5 is devoted to proving that the map (2) induces a split surjection in $K$ theory; see in particular Proposition 5.1 To prove this we will construct a transfer map going essentially the other way. In fact the target of the transfer map will not be $\mathcal{C}^{\Gamma}\left(S \mathbb{H}_{\{0\}} \tilde{M} \times \mathbb{T}, \mathcal{E}_{\text {asy }}\right)^{\infty}$ but a formally enlarged version of that category which 
yields the same $K$-theory. The construction of the transfer map will depend on the choice of a sequence $\mathbf{d}=\left(\delta^{0}, \delta^{1}, \delta^{2} \ldots\right)$ of decay speed functions which will be responsible for the decay speed one can achieve in the target. The geometry of our situation enters in verifying that a suitable sequence of decay speed functions exist; compare Lemma 5.8. The map on the $K$-theory of $\mathcal{C}^{\Gamma}\left(\tilde{M} \times \mathbb{T}, \mathcal{E}_{d}\right)^{\infty}$ induced by the composition of the transfer and the projection is described by an element in a Swan group in Proposition 5.9. In order to achieve that this element is the identity in our case, we have to work with the subbundle $S^{+} \mathbb{H}_{\{0\}} \tilde{M}$ of $S \mathbb{H}_{\{0\}} \tilde{M}$ whose fiber is contractible. This explains the necessity of the hyperbolic enlargement: it allows us to pick out this subbundle.

(3) In Section 4 we will study the map (3) which is induced by

$$
\begin{aligned}
S \mathbb{H} \tilde{M} \times \mathbb{T} & \rightarrow S \mathbb{H} \tilde{M} \times \mathbb{T} \\
(v, t) & \mapsto\left(\Phi_{t}(v), t\right),
\end{aligned}
$$

where $\Phi$ denotes the geodesic flow on $S \mathbb{H} \tilde{M}$. Via this map one gains control in the directions transverse to the geodesic flow. We prove in Theorem 4.9 that the map turns foliated control with respect to the asymptotic foliation into foliated control with respect to the geodesic foliation. In fact in the target we will have foliated control (with respect to the geodesic foliation) with exponential decay speed (depending on the upper bound for the sectional curvature), and our choice of decay speed $\mathcal{S}_{a s y}$ for $\mathcal{E}_{a s y}$ in (2) is made in such a way that we achieve this. Since $\Phi_{t}\left(S \mathbb{H}_{\{0\}} \tilde{M}\right) \subseteq S \mathbb{H}_{[-t, t]} \tilde{M}$ all objects in the image will lie in $(S \mathbb{H} \tilde{M} \times \mathbb{T})_{\llcorner}$.

(4) The bended arrow (4) is induced by the projection $S \mathbb{H} \tilde{M} \rightarrow \tilde{M}$. This collapses the non-compact $\mathbb{H}$-direction and is therefore not a proper map. In general, non-proper maps do not induce functors on our categories of modules over a space. (Such a map does not preserve the local finiteness condition; compare 2.1.2) However, the restriction of the projection to $(S \mathbb{H} \tilde{M} \times \mathbb{T}) \angle$ is proper, and we obtain a well-defined functor on objects. Since $S \mathbb{H} \tilde{M} \rightarrow \tilde{M}$ does not increase distances and $\mathcal{E}_{g e o}$-controlled morphisms are in particular bounded with respect to the product metric we obtain a well-defined functor. In Corollary 5.10 we show that the triangle induced in $K$-theory by the maps (2), (3) and (4) commutes up to homotopy. Here we use a variant of the Lipschitz homotopy argument from [HPR97].

(5) The map (5) is induced by the inclusion of $S \mathbb{H} \tilde{M} \times\{0\} \times \mathbb{T}$ into $S \mathbb{H} \tilde{M} \times$ $\mathbb{B} \times \mathbb{T}$. This inclusion is clearly compatible with the $\angle$-subspaces. We equip $\mathbb{B}$ with the standard (Euclidean) Riemannian metric and $S \mathbb{H} \tilde{M} \times \mathbb{B}$ with the product Riemannian structure. Also we extend the geodesic foliation (by taking the product with the trivial 0-dimensional foliation of $\mathbb{B}$ ) to a foliation $F_{w}$ of $S \mathbb{H} \tilde{M} \times \mathbb{B}$. Now $\mathcal{E}_{w}$ is defined similarly to $\mathcal{E}_{g e o}$ as foliated control with a certain carefully chosen decay speed $\mathcal{S}$ (defined in Subsection 6.3) with respect to the foliation $F_{w}$. It will follow from the construction (see Proposition 6.13 (i) that the inclusion maps $\mathcal{E}_{g e o}$-control to $\mathcal{E}_{w}$-control. The $\mathcal{F}_{\mathbb{B}}$-object support condition consists of all subsets whose projection to $\mathbb{B}$ is contained in a compact interval $\left[0, \beta_{0}\right]$.

(6) The map (6) is induced by the projection $S \mathbb{H} \tilde{M} \times \mathbb{B} \rightarrow \tilde{M}$. As in (4) this projection is not proper since it collapses the non-compact $\mathbb{H}$ - and $\mathbb{B}$-directions. 
However the restriction to the $\angle$-subspace and the $\mathcal{F}_{\mathbb{B}}$ condition ensure that we nevertheless have a well-defined functor on objects. By construction $\mathcal{E}_{w}$-control dominates metric control and the projection does not increase distances. Therefore (6) is also compatible with the morphism control conditions.

(7) The fact that the map (7) induces an equivalence in $K$-theory should be thought of as a "foliated control theorem". It is proven as Theorem 6.17. Very roughly, this theorem improves foliated control to metric control (with decay speed) on compact subsets (in the $S \mathbb{H} \tilde{M}$-coordinate) that do not meet preimages of "short" closed geodesics in $M$. The relatively long Section [ 6 is devoted to the proof of this theorem. The only difference between source and target of the map (7) are the morphism support conditions. As explained above, the weak control condition $\mathcal{E}_{w}$ is essentially, i.e. up to the added $\mathbb{B}$-direction, foliated control with respect to the geodesic foliation with a certain carefully chosen decay speed $\mathcal{S}$. The stronger control condition $\mathcal{E}_{s}$ is obtained from $\mathcal{E}_{w}$ by adding a metric control condition with decay speed $\mathcal{S}$ over a certain subset $S \subset S \mathbb{H} \tilde{M} \times \mathbb{B} \times \mathbb{T}$. The subset and the precise control condition will be explained in Subsection 6.4.

(8) The map (8) is induced from the projection $p_{X}: S \mathbb{H} \tilde{M} \rightarrow X$. In particular this map collapses the non-compact $\mathbb{H}$-direction, and hence the remaining $\mathcal{F}_{\mathbb{B}}$-object support in the target is just the usual $\Gamma$-compact support. The $\mathcal{E}_{s}$-condition is shown to be strong enough to induce a map in Proposition 7.1 .

The lower left-hand arrow is an equality which spells out the definition given in Subsection [2.2.2.

This finishes the outline of the proof.

We start the proof by the following easy lemma about the map (1).

Lemma 3.1. The map (1) induces an equivalence in $K$-theory.

Proof. The map $\mathcal{C}^{\Gamma}\left(\tilde{M} \times \mathbb{T}, \mathcal{E}_{d}\right) \rightarrow \mathcal{C}^{\Gamma}\left(\tilde{M} \times \mathbb{T}, *^{-1} \mathcal{E}_{\Gamma c c}\right)$ induces a map between the two corresponding "germs at infinity" fibrations; see Example 8.8. In the resulting ladder the two middle terms allow an Eilenberg swindle towards infinity along $\mathbb{T}$ (compare [BFJR04, 4.4, 4.5]) and the left-hand terms are even equal.

\section{Gaining control via the Geodesic flow}

There are two foliations on $S \mathbb{H} \tilde{M}$, the geodesic foliation and the asymptotic foliation. In this section we will define the notion of foliated control with a prescribed decay speed and we will prove in Theorem 4.9 that the geodesic flow can be used to turn foliated control with a certain decay speed with respect to the asymptotic foliation into foliated control with exponential decay speed with respect to the geodesic foliation. Finally we show that after forgetting control the map induced by the geodesic flow is homotopic to the identity (more precisely to a certain inclusion); see Theorem 4.11

4.1. Geometric preparations. Recall from Section 3 that $\mathbb{H} \tilde{M}$ denotes the hyperbolic enlargement and $S \mathbb{H} \tilde{M}$ its sphere bundle. The space $S \mathbb{H} \tilde{M}$ will be equipped with two foliations, the geodesic foliation and the asymptotic foliation. Let $\Phi: \mathbb{R} \times$ 
$S \mathbb{H} \tilde{M} \rightarrow S \mathbb{H} \tilde{M},(t, v) \mapsto \Phi_{t}(v)$ denote the geodesic flow. The geodesic foliation $F_{\text {geo }}$ is simply the 1-dimensional foliation given by the flow lines of $\Phi$. Two points $v$ and $w$ in $S \mathbb{H} \tilde{M}$ are called asymptotic if the distance between $\Phi_{t}(v)$ and $\Phi_{t}(w)$ stays bounded if $t$ tends to $+\infty$. This defines an equivalence relation, and the set of equivalence classes, denoted $S(\infty)$, can be naturally equipped with a topology in such a way that the map $a: S \mathbb{H} \tilde{M} \rightarrow S(\infty)$ given by sending a vector to its equivalence class restricts to a homeomorphism on each fiber $S \mathbb{H} \tilde{M}_{x}$ of the bundle $S \mathbb{H} \tilde{M} \rightarrow \mathbb{H} \tilde{M}$; compare Section 1 in [EO73]. The preimages $a^{-1}(\theta)$ for $\theta \in S(\infty)$ are the leaves of a foliation $F_{a s y}$, which we will call the asymptotic foliation. Since $M$ is compact there are positive constants $a$ and $b$ such that the sectional curvature $K$ satisfies

$$
-b^{2} \leq K \leq-a^{2}
$$

The same inequalities hold for the hyperbolic enlargement; compare Lemma 2.1 (vi) in FJ86.

The homeomorphisms $S \mathbb{H} \tilde{M}_{x} \rightarrow S(\infty)$ are used to define the fiber transport

$$
\nabla_{y, x}: S \mathbb{H} \tilde{M}_{x} \cong S(\infty) \cong S \mathbb{H} \tilde{M}_{y}
$$

for the bundle $S \mathbb{H} \tilde{M} \rightarrow \mathbb{H} \tilde{M}$. The fiber transport will play an important role in Section 5. Since we have curvature bounds the fiber transport is known to be Hölder continuous. More precisely a consequence of Proposition 2.1 in AS85] is the following lemma which will be used in Theorem 4.9 and Lemma 5.8 .

Lemma 4.2. For all $\alpha>0$ there is a constant $C_{0}(\alpha)>0$ such that for $x, y \in \mathbb{H} \tilde{M}$ with $d(x, y) \leq \alpha$ and $v, w \in S \mathbb{H} \tilde{M}_{x}$ we have

$$
d\left(\nabla_{y, x}(v), \nabla_{y, x}(w)\right) \leq C_{0}(\alpha) \cdot d(v, w)^{\frac{a}{b}} .
$$

Later we will have to quantitatively analyze how the flow $\Phi_{t}: S \mathbb{H} \tilde{M} \rightarrow S \mathbb{H} \tilde{M}$ may increase distances. For this purpose we introduce the function $C_{f l w}(t)$ in the following lemma.

Lemma 4.3. There exists a monotone increasing function $C_{\text {flw }}(t)$ such that $\left|d \Phi_{s}\right|$ $\leq C_{\text {flw }}(t)$ for all $|s| \leq t$. In particular for arbitrary $v, w \in S \mathbb{H} \tilde{M}$ we have

$$
d\left(\Phi_{t}(v), \Phi_{t}(w)\right) \leq C_{f l w}(t) \cdot d(v, w) .
$$

Proof. This again holds since we have curvature bounds. The differential of the geodesic flow can be expressed in terms of Jacobi fields; compare [EHS93, Section 2.3]. These satisfy a second-order differential equation involving the sectional curvature as coefficients [CE75 pp. 15-16], and the result can be deduced from this equation using standard arguments about ordinary differential equations; compare e.g. [Per01, p. 79].

4.2. Foliated control with decay speed. We now want to define the notions of metric respectively foliated control with decay speed $\mathcal{S}$. Let $F$ be a foliation of a Riemannian manifold $N$. For $x, y \in N$ we will write

$$
d_{F}(x, y) \leq(\alpha, \delta)
$$

if there is a piecewise $C^{1}$-path of arclength shorter than $\alpha$ which is entirely contained in one leaf of the foliation and whose start- respectively end-point lies within distance $\frac{\delta}{2}$ of $x$ respectively $y$. (In BFJR04 we used $\delta$ instead of $\frac{\delta}{2}$, but compare 
Remark 4.5) Suppose we are given a set $\mathcal{S}$ of functions from $\mathbb{T}$ to $[0, \infty$ ). (We often use $\delta_{t}$ as the name for the function which sends $t$ to $\delta_{t}$.) Suppose $\mathcal{S}$ satisfies the following conditions.

(A) For each $\delta_{t} \in \mathcal{S}$ and every $\alpha \in \mathbb{R}$ there exists $\delta_{t}^{\prime}$ and $t_{0} \geq 1$ such that $\delta_{t+\alpha} \leq \delta_{t}^{\prime}$ for all $t \geq t_{0}+|\alpha|$.

(B) Given $\delta_{t}, \delta_{t}^{\prime} \in \mathcal{S}$ there exists $\delta_{t}^{\prime \prime} \in \mathcal{S}$ and $t_{0} \geq 1$ such that $\delta_{t}+\delta_{t}^{\prime} \leq \delta_{t}^{\prime \prime}$ for all $t \geq t_{0}$.

Given such a set of functions $\mathcal{S}$ we make the following definitions.

Definition 4.4 (Foliated and metric control with decay speed).

(i) Suppose $X=(X, d)$ is a metric space. Then we define a morphism support condition $\mathcal{E}=\mathcal{E}(X, \mathcal{S})$ on $X \times \mathbb{T}$ by requiring that a subset $E$ of $(X \times \mathbb{T})^{\times 2}$ belongs to $\mathcal{E}$ if there exists a $\delta_{t} \in \mathcal{S}$ and constants $\alpha>0$ and $t_{0}>1$ such that for all $\left(x, t, x^{\prime}, t^{\prime}\right) \in E$ we have

$$
\left|t-t^{\prime}\right| \leq \alpha, \quad d\left(x, x^{\prime}\right) \leq \alpha
$$

and if $t, t^{\prime}>t_{0}$, then

$$
d\left(x, x^{\prime}\right) \leq \delta_{\min \left(t, t^{\prime}\right)} .
$$

This condition will be called metric control with decay speed $\mathcal{S}$.

(ii) Suppose $N$ is a Riemannian manifold equipped with a foliation $F$. We define $\mathcal{E}=\mathcal{E}(N, F, \mathcal{S})$, a set of subsets of $(N \times \mathbb{T})^{\times 2}$, by requiring that a subset $E$ belongs to $\mathcal{E}$ if there exists a function $\delta_{t} \in \mathcal{S}$ and constants $\alpha>0$ and $t_{0}>1$ such that for all $\left(x, t, x^{\prime}, t^{\prime}\right) \in E$ we have

$$
\left|t-t^{\prime}\right| \leq \alpha, \quad d\left(x, x^{\prime}\right) \leq \alpha
$$

and if $t, t^{\prime}>t_{0}$, then

$$
d_{F}\left(x, x^{\prime}\right) \leq\left(\alpha, \delta_{\min \left(t, t^{\prime}\right)}\right) .
$$

If $\mathcal{E}$ defines a morphism-control condition, then it will be called foliated control with respect to the foliation $F$ with decay speed $\mathcal{S}$.

Remark 4.5. Observe that for $\alpha=0$ the foliated condition in (ii) specializes to the metric condition in (i); i.e., if $\alpha=0$, then an $\left(\alpha, \delta_{t}\right)$-foliated controlled morphism is $\delta_{t}$-controlled in the metric sense.

Remark 4.6. Note that these definitions only depend on the behaviour of functions in $\mathcal{S}$ in a neighborhood of $\infty$; i.e, what is really important about $\mathcal{S}$ is the set of germs (at infinity) of functions it determines.

Warning 4.7. While conditions (A) and (B) guarantee that in the metric case $\mathcal{E}(X, \mathcal{S})$ is a morphism-control condition, in general this may fail in the foliated case for $\mathcal{E}=\mathcal{E}(N, F, \mathcal{S})$ because it is not clear that $\mathcal{E}$ is closed under composition as is required in [BFJR04, (i) on p. 167]. However in the two cases we are interested in $\mathcal{E}$ is closed under composition by Lemma 4.10 combined with Lemma 4.8 (ii) below. 


\subsection{Gaining control via the geodesic flow. Now set}

$$
\begin{aligned}
& \mathcal{S}_{\text {geo }}=\{A \cdot \exp (-a t) \mid A>0\}, \\
& \mathcal{S}_{\text {asy }}=\left\{A \cdot \exp \left(-\lambda\left(C_{\text {flw }}(2 t+B)+(t+B)^{2}\right)\right) \mid A>0, B \in \mathbb{R}, \lambda>0\right\},
\end{aligned}
$$

where in the first line $a$ comes from the upper curvature bound in (4.1). In the second line we take $C_{f l w}(2 t+B)=C_{f l w}(0)$ for $2 t+B<0$. Clearly conditions (A) and (B) before Definition 4.4 are satisfied in both cases. Here $\mathcal{S}_{a s y}$ is designed in such a way that the geodesic flow will turn $\mathcal{S}_{a s y}$-decay speed into $\mathcal{S}_{\text {geo }}$-decay speed; see Theorem 4.9 which uses property (i) of the following elementary lemma.

Lemma 4.8. Apart from (A) and (B) before Definition 4.4, $\mathcal{S}_{\text {asy }}$ satisfies:

(i) For $\delta_{t} \in \mathcal{S}_{\text {asy }}$ and each $\alpha>0$ there exists a $\delta_{t}^{\prime} \in \mathcal{S}_{\text {geo }}$ and $t_{0} \geq 1$ such that

$$
C_{f l w}(t+\alpha) \cdot \delta_{t} \leq \delta_{t}^{\prime} \text { for all } t \geq t_{0} .
$$

(ii) If $\delta_{t} \in \mathcal{S}_{\text {asy }}$ and $\lambda>0$, then $\left(\delta_{t}\right)^{\lambda} \in \mathcal{S}_{\text {asy }}$.

We now define $\mathcal{E}_{\text {geo }}$ to be foliated control with exponential decay speed $\mathcal{S}_{\text {geo }}$ with respect to the geodesic foliation $F_{\text {geo }}$ and $\mathcal{E}_{\text {asy }}$ to be foliated control with decay speed $\mathcal{S}_{a s y}$ with respect to the asymptotic foliation $F_{a s y}$, i.e.

$$
\begin{aligned}
\mathcal{E}_{\text {geo }} & =\mathcal{E}\left(S \mathbb{H} \tilde{M}, F_{\text {geo }}, \mathcal{S}_{\text {geo }}\right), \\
\mathcal{E}_{\text {asy }} & =\mathcal{E}\left(S \mathbb{H} \tilde{M}, F_{\text {asy }}, \mathcal{S}_{\text {asy }}\right) .
\end{aligned}
$$

We are now prepared to formulate the main result of this section. Recall that $(S \mathbb{H} \tilde{M} \times \mathbb{T})_{<}$is the subspace given by $|h| \leq t$, where $h$ denotes the $\mathbb{H}$-coordinate and $t$ the $\mathbb{T}$-coordinate.

Theorem 4.9. The map $(v, t) \mapsto\left(\Phi_{t}(v), t\right)$ on $S \mathbb{H} \tilde{M} \times \mathbb{T}$ turns $\mathcal{E}_{\text {asy }}$-control into

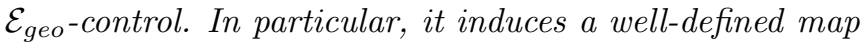

$$
\mathcal{C}^{\Gamma}\left(S \mathbb{H}_{\{0\}} \tilde{M} \times \mathbb{T}, \mathcal{E}_{a s y}\right)^{\infty} \rightarrow \mathcal{C}^{\Gamma}\left((S \mathbb{H} \tilde{M} \times \mathbb{T})_{\measuredangle}, \mathcal{E}_{\text {geo }}\right)^{\infty} .
$$

Proof. We recall some results which were discussed in BFJR04] in Proposition 14.2 and Lemma 14.3 and rely on HIH77. Let $a$ and $b$ be the curvature constants from (4.1). With the constants $C=\left(1+b^{2}\right)^{1 / 2}, D=1 / a$ and the function $E(\alpha)=$ $\frac{2}{b} \sinh \left(\frac{b}{2}\left(\alpha+\frac{1}{a}\right)\right)$ we have for any pair $v, w \in S \mathbb{H} \tilde{M}$ of asymptotic vectors with $d(v, w) \leq \alpha$ the following inequality:

$$
d_{F_{\text {geo }}}\left(\Phi_{t}(v), \Phi_{t^{\prime}}(w)\right) \leq\left(C \cdot\left(\alpha+\left|t-t^{\prime}\right|+D\right), C \cdot 2 E(\alpha) \cdot e^{-a t}\right) .
$$

Now consider $\left(v, t, w, t^{\prime}\right)$, where $v$ and $w$ are no longer assumed to be asymptotic. Assume $t^{\prime}>t$. Then we have because of Lemma 4.3 for any monotone decreasing function $\delta_{t}$ that

implies

$$
d_{F_{a s y}}(v, w) \leq\left(\alpha, \delta_{t}\right) \text { and } t^{\prime}-t \leq \alpha
$$

$$
d_{F_{g e o}}\left(\Phi_{t}(v), \Phi_{t^{\prime}}(w)\right) \leq\left(C \cdot(2 \alpha+D), C \cdot 2 E(\alpha) \cdot e^{-a t}+C_{f l w}(t+\alpha) \delta_{t}\right) .
$$

By Lemma 4.8 (i) and property (B) for $\mathcal{S}_{\text {geo }}$ this implies the claim about the morphism control conditions. Since the flow-speed in the $\mathbb{H}$-direction is at most 1 we see that $(v, t) \mapsto\left(\Phi_{t}(v), t\right)$ maps $S \mathbb{H}_{\{0\}} \tilde{M} \times \mathbb{T}$ to $(S \mathbb{H} \tilde{M} \times \mathbb{T})_{L \text {. }}$

We still have to verify that $\mathcal{E}_{\text {geo }}$ and $\mathcal{E}_{a s y}$ are well-defined morphism-support conditions; compare Warning 4.7] This is a consequence of Lemma 4.8 and the following lemma. 
Lemma 4.10 (Foliated triangle inequalities). For $u, v, w \in S \mathbb{H} \tilde{M}$ we have:

(i) If $d_{F_{g e o}}(u, v) \leq(\alpha, \delta)$ and $d_{F_{g e o}}(v, w) \leq(\beta, \epsilon)$, then

$$
d_{F_{g e o}}(u, w) \leq\left(\alpha+\beta, \delta+C_{f l w}(\alpha)(\epsilon+\delta)+\epsilon\right) .
$$

(ii) If $d_{F_{a s y}}(u, v) \leq(\alpha, \delta)$ and $d_{F_{a s y}}(v, w) \leq(\beta, \epsilon)$, then

$$
d_{F_{a s y}}(u, w) \leq\left(C \alpha+C\left(\frac{\delta+\epsilon}{2}\right)+\beta, \delta+2 C_{0}\left(\alpha+\frac{\delta+\epsilon}{2}\right)(C+1)^{\frac{a}{b}}\left(\frac{\delta+\epsilon}{2}\right)^{\frac{a}{b}}+\epsilon\right) .
$$

Here the constant $C_{0}$ stems from Lemma 4.2 and $C$ from the proof of Theorem 4.9 .

Proof. In both cases there are $u_{0}, v_{0}, v_{1}$ and $w_{1}$ within distance $\delta$ respectively $\epsilon$ from $u, v$ respectively $w$ such that $u_{0}$ and $v_{0}$ respectively $v_{1}$ and $w_{1}$ can be joined by a curve of length $\alpha$ respectively $\beta$ contained in a leaf of the foliation in question. For the first statement assume that $\Phi_{t}\left(u_{0}\right)=v_{0}$ and let $\bar{u}_{0}=\Phi_{-t}\left(v_{1}\right)$. Then $\bar{u}_{0}$ and $w_{1}$ are contained in a leaf of $F_{g e o}$ and can be used to prove the first inequality (using 4.3). For the second statement let $x$ and $y$ denote the foot points of $u_{0}$ and $v_{1}$ and set $\bar{u}_{0}=\nabla_{x, y}\left(v_{1}\right)$. Then $\bar{u}_{0}$ and $w_{1}$ are contained in a leaf of $F_{a s y}$ and can be used to prove the second inequality (using 4.2 and [BFJR04, 14.3]).

We finish this section by comparing the map induced by the flow to the map induced by the inclusion $S \mathbb{H}_{\{0\}} \tilde{M} \rightarrow S \mathbb{H} \tilde{M}$. This is only possible after relaxing the control conditions in the target. We denote by $\mathcal{E}_{d}$ the control condition coming from the product metric on $S \mathbb{H} \tilde{M} \times \mathbb{T}$.

Theorem 4.11. The map $S \mathbb{H}_{\{0\}} \tilde{M} \times \mathbb{T} \rightarrow(S \mathbb{H} \tilde{M} \times \mathbb{T})_{\llcorner}$defined by $(v, t) \mapsto$ $\left(\Phi_{t}(v), t\right)$ and the inclusion induce homotopic maps

$$
\mathcal{C}^{\Gamma}\left(S \mathbb{H}_{\{0\}} \tilde{M} \times \mathbb{T}, \mathcal{E}_{a s y}\right)^{\infty} \rightarrow \mathcal{C}^{\Gamma}\left((S \mathbb{H} \tilde{M} \times \mathbb{T})_{\llcorner}, \mathcal{E}_{d}\right)^{\infty}
$$

on the level of $K$-theory.

Proof. Let us abbreviate the two categories above by $\mathcal{C}_{0}$ and $\mathcal{C}_{1}$. Let $\hat{Z} \subset S \mathbb{H}_{\{0\}} \tilde{M} \times$ $[1, \infty) \times \mathbb{T}$ consist of all $(v, s, t)$ with $s \leq t$. Let $p: \hat{Z} \rightarrow S \mathbb{H}_{\{0\}} \tilde{M} \times \mathbb{T}$ denote the obvious projection. We will use $\hat{\mathcal{C}}=\mathcal{C}^{\Gamma}(\hat{Z}, \hat{\mathcal{E}})^{\infty}$. Here $\hat{\mathcal{E}}=\mathcal{E}_{d} \cap p^{-1} \mathcal{E}_{\text {asy }}$ where $\mathcal{E}_{d}$ denotes metric control with respect to a product metric on $\hat{Z}$. The arguments used in the proof of Theorem 4.9 can also be used to check that $(v, s, t) \mapsto\left(\Phi_{s}(v), t\right)$ induces a functor $H: \hat{\mathcal{C}} \rightarrow \mathcal{C}_{1}$. Moreover, $(v, t) \mapsto(v, 1, t)$ and $(v, t) \mapsto(v, t, t)$ induce functors $I, J: \mathcal{C}_{0} \rightarrow \hat{\mathcal{C}}$ while $(v, s, t) \mapsto(v, t)$ induces $P: \hat{\mathcal{C}} \rightarrow \mathcal{C}_{0}$. The claim of the theorem is that $H \circ I$ and $H \circ J$ induce equivalent maps in $K$-theory. Clearly, $P \circ I=P \circ J=\mathrm{id}_{\mathcal{C}_{0}}$. It is now sufficient to show that $I$ induces an isomorphism in $K$-theory, since then $I \circ P=\operatorname{id}_{\hat{\mathcal{C}}}$ and $H \circ I=H \circ I \circ P \circ J=H \circ J$ in $K$-theory. Now $I: \mathcal{C}_{0} \rightarrow \hat{\mathcal{C}}$ is equivalent to a Karoubi filtration with quotient $\mathcal{C}^{\Gamma}(\hat{Z}, \hat{\mathcal{E}})^{>\mathcal{F}_{q}}$, where $\mathcal{F}_{q}$ consists of all sets of the form $\{(v, s, t) \mid s \leq t$ and $(s \leq N$ or $t \leq N)\}$ for some $N$. We claim that this category is flasque. Indeed, the map $(v, s, t) \mapsto$ $(v, s-1, t)$ induces an Eilenberg swindle on it. A little care is needed in producing the swindle from this map, since it not well-defined on $(v, s, t)$ for $s<2$ (because then $s-1 \notin[1, \infty))$. However, in the quotient category in question, modules over $S \mathbb{H}_{\{0\}} \tilde{M} \times[1,2] \times \mathbb{T}$ can be ignored. Compare 2.1.6.

Corollary 4.12. The triangle consisting of the maps (3), (4) and (2) in the main diagram of Section 3 commutes up to homotopy after applying $K$-theory. 
Proof. Compose the maps in Theorem 4.11 with the map induced by the projection $S \mathbb{H} \tilde{M} \times \mathbb{T} \rightarrow \tilde{M} \times \mathbb{T}$.

\section{THE TRANSFER}

Our aim in this section is to prove that the map (2) in our main diagram in Section 3 induces a split surjective map in $K$-theory. We define $S^{+} \mathbb{H} \tilde{M}$ to be the subbundle of the sphere bundle $S \mathbb{H} \tilde{M} \subset T \mathbb{H} \tilde{M}=T \mathbb{R} \times T \tilde{M}=\mathbb{R} \times T \tilde{M}$ consisting of all vectors with non-negative $\mathbb{R}$-coordinate. Note that the fiber of this subbundle is a disk and hence contractible. This is important, because we will show below that the transfer on a bundle whose fiber has a non-trivial homotopy type is in general not a splitting of the bundle projection; see Proposition [5.9. Since the projection $S^{+} \mathbb{H}_{\{0\}} \tilde{M} \rightarrow \tilde{M}$ factorizes as

$$
S^{+} \mathbb{H}_{\{0\}} \tilde{M} \longrightarrow S \mathbb{H}_{\{0\}} \tilde{M} \longrightarrow \tilde{M}
$$

surjectivity of the map (2) is implied by the following proposition.

Proposition 5.1. The map

$$
\mathcal{C}^{\Gamma}\left(S^{+} \mathbb{H}_{\{0\}} \tilde{M} \times \mathbb{T}, \mathcal{E}_{\text {asy }}\right)^{\infty} \rightarrow \mathcal{C}^{\Gamma}\left(\tilde{M} \times \mathbb{T} ; \mathcal{E}_{d}\right)^{\infty}
$$

induced by the bundle projection induces a split surjective map in $K$-theory.

In order to prove this proposition we will produce a transfer map in the reverse direction. In fact we will construct the following (non-commutative) diagram:

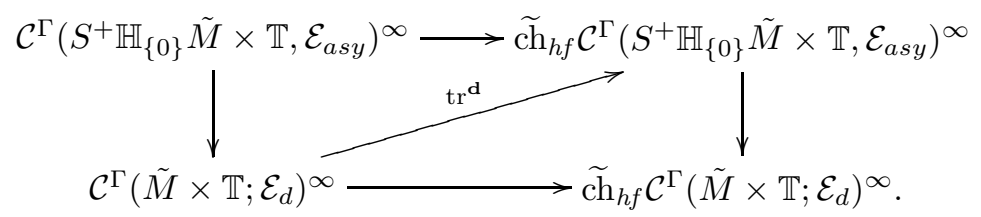

The diagonal arrow is the promised transfer. It will depend upon the choice of a sequence $\mathbf{d}=\left(\delta^{0}, \delta^{1}, \delta^{2}, \ldots\right)$ of decay speed function from $\mathcal{S}_{a s y}$. The horizontal arrows induce equivalences in $K$-theory and the square without the transfer map commutes. In Subsection [5.5 we will show that the lower triangle commutes in $K$-theory up to multiplication by a certain element in a Swan group which is determined by the homology of the fiber of the bundle $S^{+} \mathbb{H}_{\{0\}} \tilde{M}$. Since the fiber of this bundle is a disk we know that the triangle induces a commutative triangle; see Corollary 5.10.

Remark 5.3. In the diagram above and in the proof below we have to deal with certain Waldhausen categories (categories with cofibrations and weak equivalences Wal85]) which are categories of chain complexes. There seems to be no good definition of non-connective $K$-theory in the literature which applies in this generality. However in our situation we can always make an ad-hoc construction of a nonconnective $K$-theory spectrum as follows. Let $X$ be a free $\Gamma$-space and $\mathcal{E}$ a control condition on $X$. Let $\mathcal{E}_{d}$ be the standard Euclidean metric control condition on $\mathbb{R}^{n}$. Let $p: \mathbb{R}^{n} \times X \rightarrow \mathbb{R}^{n}$ and $q: \mathbb{R}^{n} \times X \rightarrow X$ be the projections and $\mathbb{K}(-)$ be Waldhausen's connective $K$-theory functor which applies to Waldhausen categories. It is well known that the spaces

$$
\mathbb{K} \mathcal{C}^{\Gamma}\left(\mathbb{R}^{n} \times X ; p^{-1} \mathcal{E}_{d} \cap p^{-1} \mathcal{E}\right)
$$


together with structure maps derived from swindles coming from a decomposition $\mathbb{R}^{n}=\mathbb{R}_{+}^{n} \cup \mathbb{R}_{-}^{n}$ yield a model for the non-connective $K$-theory spectrum of $\mathcal{C}^{\Gamma}(X ; \mathcal{E})$ (compare the last page in CP97). The same construction applies to categories such as $\operatorname{ch}_{f} \mathcal{C}(X ; \mathcal{E})$ and $\operatorname{ch}_{h f} \mathcal{C}(X ; \mathcal{E})$ and all variants which will be used below. Hence in each case it makes sense to talk of the non-connective $K$-theory. In all constructions and arguments below the $\mathbb{R}^{n}$-factor will play the role of a dummy variable. In order to facilitate the exposition we hence formulated all arguments only for the 0 -th spaces, i.e. for connective $K$-theory. It is straightforward to make the necessary modifications to obtain the analogous statement for the other spaces of the spectrum and to check compatibility with the structure maps.

5.1. Set-up. The construction of the transfer works in the following generality. Let $\tilde{B}$ be the universal covering of the compact space $B$. Let $\Gamma$ denote the fundamental group which acts on $\tilde{B}$ from the left. Let $\pi: \tilde{B} \rightarrow B$ denote the covering projection. Suppose $p: E \rightarrow B$ is a smooth fiber bundle with compact fiber. We form the pullback $\tilde{E}$ and use the following notation:

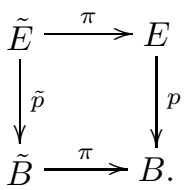

Suppose $\tilde{B}$ is equipped with a $\Gamma$-invariant metric $d$. Suppose $E$ is a Riemannian manifold and $\tilde{E}$ is equipped with the pulled-back Riemannian structure and a $\Gamma$ invariant foliation $F$. Furthermore let $\mathcal{S}$ be a set of decay speed functions (compare Subsection 4.2) and assume that $\mathcal{E}=\mathcal{E}(\tilde{E}, F, \mathcal{S})$ really defines a morphism control condition; compare Warning 4.7.

We also assume that we are given a fiber transport $\nabla$, i.e. a homeomorphism of fibers $\nabla_{b^{\prime}, b}: \tilde{E}_{b} \rightarrow \tilde{E}_{b^{\prime}}$ for each pair of points $b$ and $b^{\prime}$ in $\tilde{B}$ which fulfills the following requirements.

Assumption 5.4. The fiber transport has the following properties:

(i) It is functorial, i.e. $\nabla_{b, b}=\mathrm{id}_{\tilde{E}_{b}}$ and $\nabla_{b^{\prime \prime}, b^{\prime}} \circ \nabla_{b^{\prime}, b}=\nabla_{b^{\prime \prime}, b}$ for all $b^{\prime \prime}, b^{\prime}$ and $b \in \tilde{B}$.

(ii) It is $\Gamma$-invariant, i.e. for all $b, b^{\prime} \in \tilde{B}$ and all $g \in \Gamma$ we have

$$
l_{g} \circ \nabla_{b^{\prime}, b} \circ l_{g}^{-1}=\nabla_{g b^{\prime}, g b} .
$$

Here $l_{g}: \tilde{E}_{b} \rightarrow \tilde{E}_{g b}$ is the restriction of the left action of $\Gamma$ on $\tilde{E}$.

(iii) It is compatible with the foliation in the following strong sense. There exists a constant $C \geq 1$ such that for all $b, b^{\prime} \in \tilde{B}$ with $d\left(b, b^{\prime}\right) \leq \alpha$ and every $e \in \tilde{E}_{b}$ we have

$$
d_{\mathcal{F}}\left(\nabla_{b^{\prime}, b} e, e\right) \leq(C \alpha, 0)
$$

i.e., there is a path of length no longer than $C \alpha$ inside one leaf which connects $\nabla_{b, b^{\prime}} e$ and $e$.

An additional requirement will be formulated in Assumption 5.6 below. All the assumptions are fulfilled in our situation where $E \rightarrow B$ is the bundle $S^{+} \mathbb{H}_{\{0\}} M \rightarrow$ $M$, the foliation is the asymptotic foliation $F_{a s y}$ and $\mathcal{S}=\mathcal{S}_{\text {asy }}$. Assumption (iii) follows from [BBE85, 1.1]: if $\phi(t)$ is a geodesic in $\tilde{M}$ from $b$ to $b^{\prime}$, then the path 
$t \mapsto \nabla_{\phi(t), b} e$ is contained in a leaf of $F_{a s y}$ and is no longer than $\left(1+b^{2}\right)^{1 / 2} \cdot \alpha$, where $-b^{2}$ is a lower bound for the curvature of $M$.

5.2. Homotopy finite chain complexes. Below we would like to work with singular chain complexes, which have the advantage that they do not depend on any further choices (like triangulations or CW-structures). On the other hand, in order to define $K$-theory we need to impose some finiteness conditions; i.e., we want to work with homotopy finite chain complexes. We now introduce the necessary notation. Given a free $\Gamma$-space $X$ and a control-condition $\mathcal{E}$ we define $\overline{\mathcal{C}}^{\Gamma}(X ; \mathcal{E})$ completely analogous to $\mathcal{C}^{\Gamma}(X ; \mathcal{E})$, but we do not require that the modules are locally finite. We hence allow objects $M=\left(M_{x}\right)$ whose support $\operatorname{supp} M=\left\{x \in X \mid M_{x} \neq 0\right\}$ is an arbitrary subset of $X$. Also the free $R$-modules $M_{x}$ need not be finitely generated. (We should however require that the cardinality of the bases are bounded by some fixed large enough cardinal. This allows us to choose small models for all the categories that will appear below.) We think of the full subcategory $\mathcal{C}^{\Gamma}(X ; \mathcal{E})$ inside $\overline{\mathcal{C}}^{\Gamma}(X ; \mathcal{E})$ as the category of "finite" objects and define as explained in Appendix 8.1 the categories of finite, respectively homotopy finite chain complexes

$$
\operatorname{ch}_{f} \mathcal{C}^{\Gamma}(X ; \mathcal{E}) \quad \text { and } \quad \operatorname{ch}_{h f} \mathcal{C}^{\Gamma}(X ; \mathcal{E})
$$

Both categories are full subcategories of the category $\operatorname{ch} \overline{\mathcal{C}}^{\Gamma}(X ; \mathcal{E})$ of "all" chain complexes and are naturally equipped with the structure of a Waldhausen category; see Appendix 8.1. The natural inclusions

$$
\mathcal{C}^{\Gamma}(X ; \mathcal{E}) \rightarrow \operatorname{ch}_{f} \mathcal{C}^{\Gamma}(X ; \mathcal{E}) \rightarrow \operatorname{ch}_{h f} \mathcal{C}^{\Gamma}(X ; \mathcal{E})
$$

induce equivalences on $K$-theory; compare Lemma 8.1 and Remark 5.3. Analogous considerations apply to $\mathcal{C}^{\Gamma}(X \times \mathbb{T} ; \mathcal{E})^{\infty}$ considered as the subcategory of "finite" objects in $\overline{\mathcal{C}}^{\Gamma}(X \times \mathbb{T} ; \mathcal{E})^{\infty}$.

5.3. The fiber complex. A chain complex $C \in \operatorname{ch} \overline{\mathcal{C}}^{\Gamma}(\tilde{E} \times \mathbb{T}, \mathcal{E})$ is called a fiberwise chain complex if no differential connects different fibers; i.e., if a pair of points lies in the support of a differential, then both points lie in the same fiber of the bundle $\tilde{E} \times \mathbb{T} \rightarrow \tilde{B} \times \mathbb{T}$. Given such a fiberwise complex and a point $(b, t)$ in the base we define the fiber $C_{(b, t)}$ to be the largest subcomplex such that the support of all its modules lies in the fiber $\tilde{E} \times \mathbb{T}_{(b, t)}=\tilde{E}_{b} \times\{t\}$.

We define the fiberwise complex $F$ and for a given $\delta=\delta_{t} \in \mathcal{S}$ the fiberwise complex $F^{\delta}$ by

$$
F_{(b, t)}=C_{\text {sing }}\left(E_{\pi(b)}\right) \quad \text { and } \quad F_{(b, t)}^{\delta}=C_{\text {sing }}^{\delta_{t}}\left(E_{\pi(b)}\right) .
$$

Here $C_{\text {sing }}$ denotes the singular chain complex and $C_{\text {sing }}^{\delta_{t}}$ denotes the subcomplex generated by all singular simplices $\sigma: \Delta \rightarrow E_{\pi(b)}$ which have the property that $\sigma(\Delta)$ has diameter $\leq \delta_{t}$ in $E$. The complexes $F_{(b, t)}$ and $F_{(b, t)}^{\delta}$ are complexes over $E_{\pi(b)}$ by gluing each singular simplex to the image of its barycenter. The complexes $F$ and $F^{\delta}$ become $\Gamma$-invariant complexes over $\tilde{E} \times \mathbb{T}$ via the maps

$$
E_{\pi(b)} \stackrel{\pi}{\cong} \tilde{E}_{b} \stackrel{\cong}{\longrightarrow} \tilde{E}_{b} \times\{t\} \stackrel{\text { inc }}{\longrightarrow} \tilde{E} \times \mathbb{T}
$$


Observe that for $F^{\delta}$ the condition on the size of the singular simplices assures that each differential is $\left(0, \delta_{t}\right)$-controlled and hence $\mathcal{E}=\mathcal{E}(F, \mathcal{S})$-controlled so that $F^{\delta} \in \operatorname{ch} \overline{\mathcal{C}}^{\Gamma}(\tilde{E}, \mathcal{E})$. This is not true for the full singular chain complex $F$.

Given an $R$-module $M \in \mathcal{C}^{\Gamma}\left(\tilde{B} \times \mathbb{T}, \mathcal{E}_{d} ; R\right)$ and a fiberwise $\mathbb{Z}$-chain complex $C \in \operatorname{ch} \overline{\mathcal{C}}^{\Gamma}(\tilde{E} \times \mathbb{T}, \mathcal{E} ; \mathbb{Z})$ we define the fiberwise $R$-chain complex

$$
M \otimes C \in \operatorname{ch} \overline{\mathcal{C}}^{\Gamma}(\tilde{E} \times \mathbb{T}, \mathcal{E} ; R)
$$

by requiring that its fibers are given by $(M \otimes C)_{(b, t)}=M_{(b, t)} \otimes_{\mathbb{Z}} C_{(b, t)}$. In particular we consider the fiberwise complex $M \otimes F^{\delta}$.

Using part of Remark 5.5 below and the fact that our fibers admit arbitrarily fine triangulations one can show that for $\delta \in \mathcal{S}$ the complex $M \otimes F^{\delta}$ is homotopy equivalent inside $\operatorname{ch} \overline{\mathcal{C}}^{\Gamma}(\tilde{E} \times \mathbb{T} ; \mathcal{E})$ to a locally finite complex and hence

$$
M \otimes F^{\delta} \in \operatorname{ch}_{h f} \mathcal{C}^{\Gamma}(\tilde{E} \times \mathbb{T} ; \mathcal{E}) .
$$

Remark 5.5. Let $T$ be a triangulation of the metric space $X=|T|$ such that the diameter of each simplex is smaller than $\delta$. Let $C(T)$ denote the chain complex associated to the triangulation and let $C_{\text {sing }}^{\epsilon}(X)$ denote the subcomplex of the singular chain complex of $X$ generated by all singular simplices which are smaller than $\epsilon$. Both complexes can be considered as complexes over $X$ using the barycenters. If $0<\delta \leq \delta_{1} \leq \delta_{2}$, then there are natural inclusions

$$
C(T) \rightarrow C^{\delta_{1}}(X) \rightarrow C^{\delta_{2}}(X) .
$$

Both maps are chain homotopy equivalences, and one can show that the homotopy inverse and the homotopies can be chosen to be $10 \delta_{2}$-controlled when considered as morphisms over $X$.

5.4. The transfer functor. The discussion above suffices to define the desired transfer functor on objects. In order to define it on morphisms we need the fiber transport. For $(b, t)$ and $\left(b^{\prime}, t^{\prime}\right) \in \tilde{B} \times \mathbb{T}$ the map

$$
E_{\pi(b)} \stackrel{\cong}{\pi} \tilde{E}_{b} \stackrel{\nabla_{b^{\prime}, b}}{\longrightarrow} \tilde{E}_{b^{\prime}} \stackrel{\cong}{\underset{\pi}{\longrightarrow}} E_{\pi\left(b^{\prime}\right)}
$$

induces a chain map

$$
F_{(b, t)} \rightarrow F_{\left(b^{\prime}, t^{\prime}\right)}
$$

which we will denote by $\nabla_{(b, t),\left(b^{\prime}, t^{\prime}\right)}$. Using Assumption 5.4 (i) one checks that

$$
\begin{aligned}
M & \mapsto M \otimes F \\
f=\left(f_{\left(b^{\prime}, t^{\prime}\right),(b, t)}\right) & \mapsto f \otimes \nabla=\left(f_{\left(b^{\prime}, t^{\prime}\right),(b, t)} \otimes \nabla_{\left(b^{\prime}, t^{\prime}\right),(b, t)}\right)
\end{aligned}
$$

defines a functor to homotopy finite chain complexes over $\tilde{E} \times \mathbb{T}$ if one ignores the control condition. But since $\nabla_{b^{\prime}, b}$ can stretch simplices it does not induce a welldefined map on the singular simplices of a fixed restricted size. This means that the analogous definition with $F^{\delta}$ does not work. In order to deal with this problem we formally enlarge our category.

In Appendix 8.2 we construct for every Waldhausen category $\mathcal{W}$ satisfying some mild conditions (which are satisfied for categories of chain complexes in an additive category) a Waldhausen category $\widetilde{\mathcal{W}}$. Objects in this category are sequences

$$
C_{0} \stackrel{c_{0}}{\longrightarrow} C_{1} \stackrel{c_{1}}{\longrightarrow} C_{2} \stackrel{c_{2}}{\longrightarrow} \cdots
$$


where the $C_{i}$ are objects in $\mathcal{W}$ and all $c_{n}$ are simultaneously cofibrations and weak equivalences in $\mathcal{W}$. A morphism $f$ in the category $\widetilde{\mathcal{W}}$ is represented by a sequence $\left(f_{m}, f_{m+1}, f_{m+2}, \ldots\right)$ of morphisms in $\mathcal{W}$ which fit into a commutative diagram

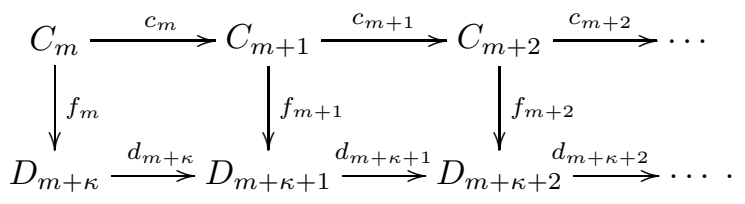

Here $m$ and $\kappa$ are non-negative integers. If we enlarge $m$ or $\kappa$ the resulting diagram represents the same morphism; i.e., we identify $\left(f_{m}, f_{m+1}, f_{m+2}, \ldots\right)$ with the sequence $\left(f_{m+1}, f_{m+2}, f_{m+3}, \ldots\right)$ but also with $\left(d_{m} \circ f_{m}, d_{m+1} \circ f_{m+1}, d_{m+2} \circ\right.$ $\left.f_{m+2}, \ldots\right)$. Sending an object to the constant sequence yields an obvious inclusion $\mathcal{W} \rightarrow \widetilde{\mathcal{W}}$ and according to Proposition 8.2 this inclusion induces an equivalence in connective $K$-theory. In the case where $\mathcal{W}=\operatorname{ch}_{h f} \mathcal{C}^{\Gamma}(\tilde{E} \times \mathbb{T} ; \mathcal{E})$ we write $\widetilde{c h}_{h f} \mathcal{C}^{\Gamma}(\tilde{E} \times \mathbb{T} ; \mathcal{E})$ for the corresponding enlargement and using Remark 5.3 we conclude that the inclusion induces an equivalence in non-connective $K$-theory.

Let $\mathbf{d}=\left(\delta^{0}, \delta^{1}, \delta^{2}, \ldots\right)$ be a monotone increasing sequence of decay speed functions $\delta^{i} \in \mathcal{S}$; i.e., for all $i \geq 0$ and all $t \in \mathbb{T}$ we have $\delta_{t}^{i} \leq \delta_{t}^{i+1}$. Then for a module $M \operatorname{in} \mathcal{C}^{\Gamma}\left(\tilde{B} \times \mathbb{T} ; \mathcal{E}_{d}\right)$

$$
M \otimes F^{\mathbf{d}}=\left(M \otimes F^{\delta^{0}} \rightarrow M \otimes F^{\delta^{1}} \rightarrow M \otimes F^{\delta^{2}} \rightarrow \ldots\right),
$$

defines an object in $\widetilde{c h}_{h f} \mathcal{C}^{\Gamma}(\tilde{E} \times \mathbb{T} ; \mathcal{E})$. Here the maps in the sequence are the natural inclusion maps. They are shown to be $\mathcal{E}$-controlled homotopy equivalences using again Remark 5.5

Assumption 5.6. Suppose for each $\alpha \geq 0$ there exists an integer $\kappa(\alpha)$ such that the following holds:

If $d\left(b, b^{\prime}\right) \leq \alpha$ and $\left|t-t^{\prime}\right| \leq \alpha$, then for all $e \in \tilde{E}_{b^{\prime}}$ we have

$$
\nabla_{b, b^{\prime}}\left(\{e\}^{\delta_{t}^{i}}\right) \subset \nabla_{b, b^{\prime}}(\{e\})^{\delta_{t^{\prime}}^{i+\kappa(\alpha)}}
$$

for all sufficiently large $t, t^{\prime}$.

Here the thickenings are taken in $\tilde{E}_{b}$ and $\tilde{E}_{b^{\prime}}$ with respect to distance in the ambient manifold.

Under this assumption we immediately obtain the following proposition.

Proposition 5.7. Suppose $\mathbf{d}$ is a sequence of decay speed functions satisfying Assumption [5.6] Then there exists a functor

$$
\operatorname{tr}^{\mathbf{d}}: \mathcal{C}^{\Gamma}\left(\tilde{B} \times \mathbb{T} ; \mathcal{E}_{d}\right) \rightarrow \widetilde{\operatorname{ch}}_{h f} \mathcal{C}^{\Gamma}(\tilde{E} \times \mathbb{T} ; \mathcal{E})
$$

which sends $f: M \rightarrow N$ to the morphism in $\widetilde{c h}_{h f} \mathcal{C}^{\Gamma}(\tilde{E} \times \mathbb{T} ; \mathcal{E})$ represented by

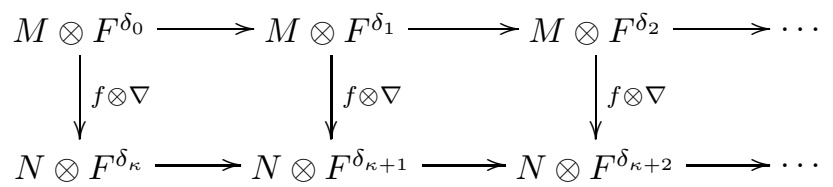

for suitably chosen $\kappa$ depending on the bound of $f$. 
It remains to check, that in our situation where the fiber bundle is $S^{+} \mathbb{H}_{\{0\}} M \rightarrow$ $M, \nabla$ is the asymptotic fiber transport and $\mathcal{S}=\mathcal{S}_{a s y}$, we can find a suitable sequence $\mathbf{d}$ of decay speed functions. The proof will use the fact that the fiber transport is Hölder continuous; compare Lemma 4.2

Lemma 5.8. There exists a sequence $\mathbf{d}=\left(\delta^{0}, \delta^{1}, \delta^{2}, \ldots\right)$ of decay speed functions $\delta^{i} \in \mathcal{S}_{\text {asy }}$ satisfying Assumption 5.6 with respect to the asymptotic fiber transport.

Proof. We abbreviate the constant $\frac{a}{b}$ appearing in Lemma 4.2 by $\lambda$. Lemma 4.2 implies that $\nabla_{b, b^{\prime}}\left(e^{\delta}\right)$ is contained in $\nabla_{b, b^{\prime}}(e)^{C_{0}(\alpha) \cdot \delta^{\lambda}}$. Thus our task is to find $\delta^{i} \in \mathcal{S}_{\text {asy }}$ such that for all $\alpha>0$ there is $\kappa(\alpha)$ such that for all sufficiently large $t, t^{\prime}$ with $\left|t-t^{\prime}\right| \leq \alpha$ we have

$$
C_{0}(\alpha)\left(\delta_{t}^{i}\right)^{\lambda} \leq \delta_{t^{\prime}}^{i}
$$

In the following it will be convenient to extend all functions on $\mathbb{T}$ to functions on $\mathbb{R}$ that are constant on $(-\infty, 1]$. Start with an arbitrary $\delta^{0} \in \mathcal{S}_{\text {asy }}$. Choose inductively $\delta^{i} \in \mathcal{S}_{\text {asy }}$ such that

$$
i \cdot\left(\delta_{t-i}^{0}+\cdots+\delta_{t-i}^{i-1}\right)^{\lambda}<\delta_{t}^{i}
$$

for all sufficiently large $t$. This is indeed possible because of Lemma 4.8 (ii) and (A) and (B) before Definition 4.4. Choose now $\kappa(\alpha) \in \mathbb{N}$ larger than $\alpha$ and $C_{0}(\alpha)$. Then

$$
C_{0}(\alpha) \cdot\left(\delta_{t}^{i}\right)^{\lambda} \leq(i+\kappa(\alpha)) \cdot\left(\delta_{t}^{i}\right)^{\lambda} \leq \delta_{t+i+\kappa(\alpha)}^{\kappa(\alpha)+i} \leq \delta_{t^{\prime}}^{\kappa(\alpha)+i},
$$

for sufficiently large $t, t^{\prime}$ with $\left|t-t^{\prime}\right| \leq \alpha$. (For the last inequality note that all functions in $\mathcal{S}_{\text {asy }}$ are monotone decreasing.)

5.5. An element in the Swan group. It remains to study the (non-commutative) triangle

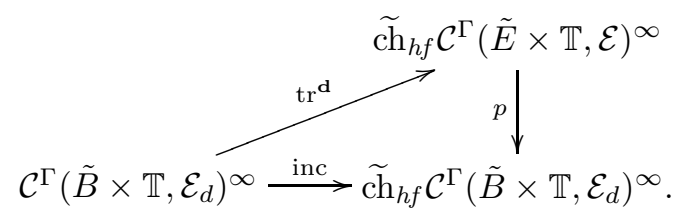

We will denote the induced maps in $K$-theory by the same symbols. Recall that the inclusion inc induces an isomorphism. What we would like to understand is the self map inc ${ }^{-1} \circ p \circ \operatorname{tr}^{\mathbf{d}}$. To describe the result we need some preparation. Let us fix a point $b_{0} \in \tilde{B}$. Observe that in general the diagram

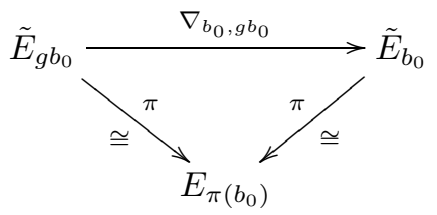

does not commute. In fact we use it to define a left $\Gamma$-operation on $E_{\pi\left(b_{0}\right)}$ by letting $g \in \Gamma$ act via $\pi \circ \nabla_{b_{0}, g b_{0}} \circ \pi^{-1}$. The singular chain complex $F_{0}=C_{\operatorname{sing}}\left(E_{\pi\left(b_{0}\right)}\right)$ hence becomes a complex of $\mathbb{Z} \Gamma$-modules. As a $\mathbb{Z}$-chain complex $F_{0}$ is homotopy equivalent to a finite complex of finitely generated $\mathbb{Z}$-modules, because the fibre $E_{\pi_{0}\left(b_{0}\right)}$ is assumed to be compact. The homology groups $H_{i}\left(F_{0}\right)$ are hence $\mathbb{Z} \Gamma$ modules which are finitely generated as $\mathbb{Z}$-modules. Such a module defines an 
element in the Swan ring $S w(\Gamma ; \mathbb{Z}) . K$-theory becomes a module over the Swan ring via the maps

$$
S w(\Gamma ; \mathbb{Z}) \otimes_{\mathbb{Z}} K_{n}(R \Gamma) \rightarrow K_{n}(R \Gamma) .
$$

The Swan ring, its action on $K$-theory and certain variants we need below in the proof are discussed in Appendix 8.3

Proposition 5.9. Under the identification

$$
K_{n}(R \Gamma) \cong K_{n+1}\left(\mathcal{C}^{\Gamma}\left(\tilde{B} \times \mathbb{T}, \mathcal{E}_{d}\right)^{\infty}\right)
$$

coming from the germs at infinity fibration (compare Example 8.8) the map inc ${ }^{-1}$ 。 $p \circ \operatorname{tr}^{\mathbf{d}}$ corresponds to multiplication with

$$
\Sigma_{i=0}^{\infty}(-1)^{i}\left[H_{i}\left(F_{0}\right)\right] \in S w(\Gamma ; \mathbb{Z}) .
$$

Corollary 5.10. Diagram (5.2) induces a commutative diagram in K-theory.

Proof. The fiber of $S^{+} \mathbb{H} \tilde{M} \rightarrow \tilde{M}$ is contractible, and hence the Swan group element is represented by the trivial $\mathbb{Z} \Gamma$-module $\mathbb{Z}$, which acts as the identity on $K$-theory.

The rest of this subsection is devoted to the proof of Proposition 5.9. Again we will only discuss the argument for connective $K$-theory. This yields Proposition 5.9 for $n \geq 1$. The general result follows by filling in extra $\mathbb{R}^{n}$-factors; compare Remark [5.3]

We first want to get rid of the ${ }^{\sim}$-construction. Consider

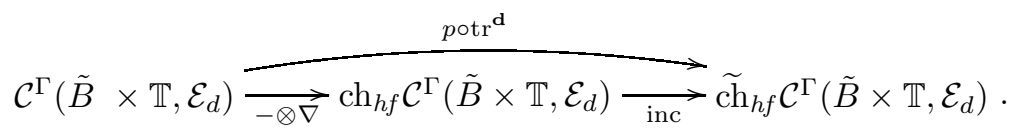

Here the functor $-\otimes \nabla$ is given by $M \mapsto M \otimes F$ and $f \mapsto f \otimes \nabla$, where now $F$ is considered as a complex over $\tilde{B} \times \mathbb{T}$ and we do not care how it is distributed over each fiber.

Lemma 5.11. There is a natural transformation between inc $\circ(-\otimes \nabla)$ and $p \circ \operatorname{tr}^{\mathbf{d}}$ which is objectwise a weak equivalence.

Proof. At $M$ the natural transformation is given by the natural inclusion

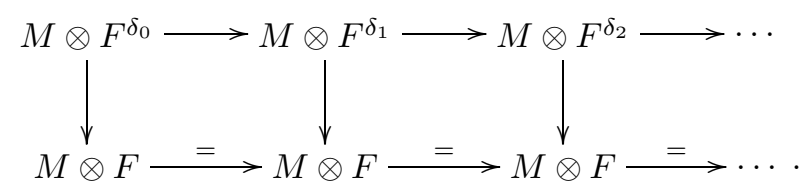

The functors and the natural transformation in Lemma 5.11 are compatible with the germs at infinity fibrations. The middle terms in these fibrations are contractible since we work with the product metric. (Compare Example 8.8 and [BFJR04, Proposition 4.4, Example 4.5] for such arguments.) Hence there is a version of Lemma 5.11 for the germs at infinity categories. We have reduced our question to comparing the two maps

$$
\mathcal{C}^{\Gamma}\left(\tilde{B}, \mathcal{E}_{d}\right) \underset{-\otimes \nabla}{\stackrel{\text { inc }}{\longrightarrow}} \operatorname{ch}_{h f} \mathcal{C} \Gamma\left(\tilde{B}, \mathcal{E}_{d}\right)
$$


Here $-\otimes \nabla$ is the obvious restriction of the functor above with the same name. Since we assume that $\tilde{B}$ is $\Gamma$-compact the $\mathcal{E}_{d}$-condition is no extra condition and we omit it in the following. For the same reason the inclusion of the orbit $\Gamma b_{0} \rightarrow \tilde{B}$ for some fixed $b_{0} \in \tilde{B}$ induces equivalences on the categories, and we are reduced to comparing the upper horizontal map in the following (non-commutative) diagram to the natural inclusion. (The diagram does commute if one replaces the horizontal maps by the natural inclusions.)

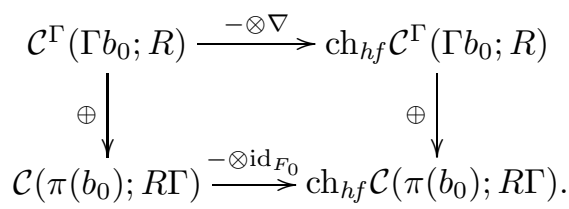

The vertical functors in this diagram are equivalences given by sending an $R$ module $M=\left(M_{g b_{0}}\right)$ over $\Gamma b_{0}$ to $\bigoplus_{g \in \Gamma} M_{g b_{0}}$ considered as an $R \Gamma$-module; compare Lemma 2.8 in BFJR04. The lower horizontal map sends an $R \Gamma$-module $N$ to the complex of $R \Gamma$-modules $N \otimes_{\mathbb{Z}} F_{0}$. Here $F_{0}$ is the singular chain complex of the fiber $E_{\pi\left(b_{0}\right)}$ considered as a $\mathbb{Z} \Gamma$-module as explained towards the beginning of this subsection and $\Gamma$ operates diagonally on $N \otimes_{\mathbb{Z}} F_{0}$. On morphisms the functor sends $f$ to $f \otimes \operatorname{id}_{F_{0}}$. Diagram (5.12) does not commute, but we have the following lemma.

Lemma 5.13. There is a natural transformation between the two ways through Diagram (5.12) which is objectwise an isomorphism.

Proof. Let $M=\left(M_{g b_{0}}\right)$ be an $R$-module over $\Gamma b_{0}$. Both ways through (5.12) send $M$ to $\left(\bigoplus_{g \in \Gamma} M_{g b_{0}}\right) \otimes F$. However, the $\Gamma$-actions are different. If we go first right and then down $\Gamma$ acts only on the first factor; if we go down and then right $\Gamma$ acts diagonally on the tensor product. The natural transformation from right/down to down/right sends $m \otimes v \in M_{g b_{0}} \otimes F_{0}$ to $m \otimes g v \in M_{g b_{0}} \otimes F_{0}$.

It remains to compare $-\otimes \mathrm{id}_{F_{0}}$ to the natural inclusion. In Appendix 8.3 we explain a variant $S w^{\mathrm{ch}}(\Gamma ; \mathbb{Z})$ of the Swan group together with its action on $K$ theory. The complex $F_{0}$ is a complex of $\mathbb{Z} \Gamma$-modules which is degreewise free as a $\mathbb{Z}$-complex and whose homology is finitely generated as an abelian group. Such a complex defines an element in $S w^{\mathrm{ch}}(\Gamma ; \mathbb{Z})$ and inc ${ }^{-1} \circ\left(-\otimes \mathrm{id}_{F_{0}}\right)$ describes the action of this element on $K$-theory. Proposition 5.9 now follows from Proposition 8.3 and the discussion following that proposition.

Remark 5.14. In the case we are interested in, where the fiber is a disk, we could avoid the Swan group and proceed differently after Lemma 5.13. In that case the augmentation $\epsilon: F_{0} \rightarrow \mathbb{Z}$ is a $\Gamma$-equivariant map which is a non-equivariant chain homotopy equivalence. In particular it induces for every free $R \Gamma$-module $N$ a homology isomorphism $N \otimes_{\mathbb{Z}} F_{0} \rightarrow N \otimes_{\mathbb{Z}} \mathbb{Z}$. Each chain module of $N \otimes_{\mathbb{Z}} F_{0}$ is non-canonically isomorphic to the $R \Gamma$-module with the same underlying abelian group but where $\Gamma$ operates only on the left tensor factor. Hence both complexes are complexes of free $R \Gamma$-modules and the homology isomorphism is in fact an $R \Gamma$ chain homotopy equivalence. This yields a natural transformation between $-\otimes F_{0}$ and the inclusion which is objectwise a weak equivalence. 


\section{A foliated CONTRol theorem for higher $K$-Theory}

In this section we will show that a certain relax control map induces an equivalence in $K$-theory. Roughly speaking the map relaxes control from metric control to foliated control with respect to the geodesic foliation. Unfortunately the precise statement is more complicated, and to formulate it we need some rather lengthy preparations. The reader should right away take a look at Subsection 6.4 to get a first idea about the statement we are aiming at.

Ignoring the technicalities the argument can be summarized as follows. We know that metric control leads to homological behavior. In particular we have the long exact sequences associated to pairs of spaces in order to work inductively over the skeleta of a cell structure. One task is now to formulate and prove analogous results for foliated control using the skeleta of a long and thin cell structure. The crucial step is a "foliated excision" result that reduces the statement about the relax control map to a comparison result for a collection of long and thin cells (compare Proposition 6.24). Carefully bookkeeping the error terms one can even assume that one has a collection of long and thin cells in Euclidean space equipped with a "standard" 1-dimensional foliation. The comparison result is then easily established: an Eilenberg swindle is used to reduce the question to transversal cells. On transversal cells metric control and foliated control coincide (compare Lemma 6.26).

6.1. Flow cell structures. Let $N$ be an $n$-dimensional Riemannian manifold which is equipped with a smooth flow $\Phi$. The flow determines a one-dimensional foliation which will be called $F$. In our application $N$ will always be $S \mathbb{H} M$ (or its universal covering) equipped with the geodesic flow and the corresponding foliation $F_{g e o}$.

The following definition of a flow cell combines Definition 7.1 and Lemma 8.1 in [FJ86]. Whereas in Lemma 8.1 in [FJ86 the information about the length of a cell is contained in the map $g_{e}$ we instead require the map to roughly preserve the length and use of a long parametrizing interval $A_{e}$. Below $\mathbb{R}^{n}=\mathbb{R} \times \mathbb{R}^{n-1}$ is equipped with the standard Euclidean metric and foliated by the lines parallel to the first coordinate axis. We denote this foliation by $F_{\mathbb{R}^{n}}$. Moreover $\mu_{n}=10^{n+3}$ is the constant which depends only on the dimension that appears in Proposition 7.2 in FJ86. Recall that for a subset $Y \subset \mathbb{R}^{n}$ we denote by $Y^{(\alpha, \delta)}$ the set of all $x \in \mathbb{R}^{n}$ for which there is a $y \in Y$ such that $d_{F_{\mathbb{R}^{n}}}(x, y) \leq(\alpha, \delta)$; compare Subsection 4.2 .

Definition 6.1 ( $\beta$-flow cell). Let $\beta>0$ be given. A cell $e \subset N$ is called a $\beta$ flow cell if there exist cells $A_{e} \subset \mathbb{R}, B_{e} \subset \mathbb{R}^{n-1}$, a number $\epsilon_{e}>0$ and a smooth embedding $g_{e}:\left(A_{e} \times B_{e}\right)^{\left(\beta, \epsilon_{e}\right)} \rightarrow N$ such that:

(i) We have $g_{e}\left(A_{e} \times B_{e}\right)=e$.

(ii) The map $g_{e}$ preserves the foliation; i.e., for each $y \in \mathbb{R}^{n-1}$ the segment $\mathbb{R} \times\{y\} \cap\left(A_{e} \times B_{e}\right)^{\left(\beta, \epsilon_{e}\right)}$ is mapped to a segment of a flow line in $N$.

(iii) If $A_{e} \subset \mathbb{R}$ is not a 0 -cell, then it is an interval of length exactly $\beta$.

(iv) For all tangent vectors $v \in T\left(\left(A_{e} \times B_{e}\right)^{\left(\beta, \epsilon_{e}\right)}\right)$ which are tangential to the flow lines we have

$$
|v|<\left|d g_{e}(v)\right| \leq \mu_{n} / 5 \cdot|v| .
$$

There are two sorts of flow cells: A flow cell where $A_{e}$ is a 0 -cell will be called transversal. If $A_{e}$ is a 1-cell we call the cell $e$ a long cell. Observe that from (iii) 
and (iv) it follows that such a cell is $\beta$-long in the sense that for every $y \in B_{e}$ the segment $g_{e}\left(A_{e} \times\{y\}\right)$ has arclength strictly larger than $\beta$ (and shorter than $\left.\beta \cdot \mu_{n} / 5\right)$.

Remark 6.2. A simple compactness argument shows that for a flow cell we additionally have the following.

(v) There exists a constant $C_{e}>1$ such that for all tangent vectors $v \in$ $T\left(\left(A_{e} \times B_{e}\right)^{\left(\beta, \epsilon_{e}\right)}\right)$ we have

$$
C_{e}^{-1} \cdot|v| \leq\left|d g_{e}(v)\right| \leq C_{e} \cdot|v| .
$$

Remark 6.3. Since (iv) and (v) hold over the $\left(\beta, \epsilon_{e}\right)$-thickening of $A_{e} \times B_{e}$, foliated distances (compare Subsection 4.2) between points in the cell which are small compared to $\left(\beta, \epsilon_{e}\right)$ can be approximately determined in Euclidean space using the chart $g_{e}$. More precisely: given $z=g_{e}(v)$ and $z^{\prime}=g_{e}\left(v^{\prime}\right)$ with $z, z^{\prime} \in e$ and

$$
d_{F}\left(z, z^{\prime}\right) \leq(\alpha, \delta) \leq\left(5 \mu_{n}^{-1} \cdot \beta, C_{e}^{-1} \cdot \epsilon_{e}\right)
$$

we have

$$
d_{F_{\mathbb{R}^{n}}}\left(v, v^{\prime}\right) \leq\left(\alpha, C_{e} \delta\right)
$$

The other way around,

$$
d_{F_{\mathbb{R}}}\left(v, v^{\prime}\right) \leq(\alpha, \delta) \quad \text { implies } \quad d_{F}\left(z, z^{\prime}\right) \leq\left(\mu_{n} \cdot \alpha, C_{e} \cdot \delta\right) .
$$

A cell structure $L$ for a compact subset of $N$ all of whose cells are $\beta$-flow cells will be called a $\beta$-flow cell structure. Given a $\beta$-flow cell structure we will always fix choices of charts $g_{e}$ and constants $\epsilon_{e}$ and $C_{e}$ as in Definition 6.1 For a given cell structure $L$ we denote by $|L| \subset N$ its underlying topological space. We recall the main result of Proposition 7.2 and Lemma 8.1 in [FJ86].

Theorem 6.4. Let $n=\operatorname{dim} N$ and $\mu_{n}=10^{n+3}$. Let $N \leq \mu_{n} \beta$ denote the union of all leaves which are shorter than $\mu_{n} \beta$. For arbitrarily large $\beta$ and any compact subset $K \subset N-N \leq \mu_{n} \beta$ there exists a $\beta$-flow-cell structure $L$ with $K \subset|L|$.

Given a cell structure $L$ we denote by $L^{[k]}$ the set of all $k$-cells and by $L^{(k)}$ the set of cells of dimension less than or equal to $k$. The $k$-skeleton is $\left|L^{(k)}\right|$. We define a filtration

$$
N^{(-1)} \subset N^{(0)} \subset \cdots \subset N^{(n)}=N
$$

of $N$ as follows. Set $N^{(-1)}=\overline{N-|L|}$ and $N^{(k)}=N^{(-1)} \cup\left|L^{(k)}\right|$. Observe that a cell $e \in L^{[k]}$ may already be contained in $N^{(-1)}$ and hence does not contribute to the $k$-th filtration step. We hence define $L^{\{k\}}$ to be the set of those $k$-cells which do not lie entirely in $N^{(-1)}$. Note that such a cell $e$ can meet $N^{(-1)}$ only with its boundary, which we denote $\partial e$.

Let $\tilde{N}$ denote the universal cover of $N$ and let $\Gamma$ be the fundamental group which acts via deck transformations on $\tilde{N}$. The lifted cell structure will be denoted $\tilde{L}$, and $\tilde{N}^{(k)}$ denotes the preimage filtration of $\tilde{N}$ under the covering projection. Also we will use $\tilde{L}^{[k]}$ and $\tilde{L}^{\{k\}}$ to denote the obvious sets of cells of $\tilde{L}$. Note that a flow cell in $N$ gives rise to a whole $\Gamma$-orbit of flow cells in $\tilde{N}$ for which one can simultaneously use the constants appearing in Definition 6.1 and Remark 6.2

Roughly speaking the following proposition says that if one removes an $(\alpha, \Delta)$ thickening of the $(k-1)$-st filtration step from the $k$-th filtration step, then the remaining pieces of the interiors of the $k$-cells are at least $(\alpha, \delta)$-foliated apart from one another. This fact will later play a crucial role in Proposition 6.24. 
Proposition 6.5. Let $L$ be a $\beta$-flow cell structure for a compact subset $|L| \subset N$. There exists an $\epsilon_{L}^{\prime}>0$ and a function $\Delta_{L}(\alpha, \delta)$ defined for $0 \leq \alpha \leq \beta$ and $0<\delta<$ $\epsilon_{L}^{\prime}$ such that $\Delta_{L}(\alpha, \delta) \geq \delta$ and the following holds:

(i) Suppose $e \in \tilde{L}^{\{k\}}$ is a $k$-cell and $x \in \tilde{N}^{(k)}$. Whenever $\Delta_{L}(\alpha, \delta)$ is defined and $\Delta \geq \Delta_{L}(\alpha, \delta)$, then

$$
d_{F}\left(e-\partial e^{(\alpha, \Delta)}, x\right) \leq(\alpha, \delta)
$$

implies that $x \in e$.

(ii) For fixed $\alpha$ the function $\Delta(\alpha, \delta)$ tends monotonically to zero when $\delta$ does.

Remark 6.6. Observe that Proposition 6.5 says in particular that for two 0-cells $e$ and $e^{\prime} \in \tilde{L}^{\{0\}}$ with

$$
d_{F}\left(e, e^{\prime}\right)<\left(\beta, \epsilon_{L}^{\prime}\right)
$$

we have $e=e^{\prime}$ since $\partial e=\emptyset$ for $e \in \tilde{L}^{[0]}$.

Proof of Proposition 6.5. For $e \in \tilde{L}^{\{k\}}, \Delta>0$ and $\alpha \geq 0$ let $Y(e, \alpha, \Delta) \subset \tilde{N}$ consist of all points $x=g_{e}\left(t_{0}+t, y\right)$ where $g_{e}\left(t_{0}, y\right) \in e-\partial e^{(\alpha, \Delta)}$ and the path $\tau \mapsto g_{e}\left(t_{0}+\tau, y\right)$ for $0 \leq \tau \leq t$ (resp. $t \leq \tau \leq 0$ ) has arclength $\leq \alpha$. (If the flow has unit speed, then $Y(e, \alpha, \Delta)$ coincides with $\Phi_{[-\alpha, \alpha]}\left(e-\partial e^{(\alpha, \Delta)}\right)$.) If $\alpha \leq \beta$, then $Y(e, \alpha, \Delta)$ is disjoint from $\tilde{N}^{(k-1)}$ and every cell $e^{\prime} \in \tilde{L}^{[k]}$ unless $e=e^{\prime}$. For a long cell $e$ this is immediate from the construction (and the fact that we assume $\Delta>0$ ). To see it in the case where $e$ is a transversal cell observe that a cell in $\tilde{L}^{\{k\}}$ can meet $\tilde{N}^{(-1)}$ only at its boundary, and hence points near (but not in) $e-\partial e^{(\alpha, \Delta)}$ which lie on a flow line which meets $e-\partial e^{(\alpha, \Delta)}$ must lie in the interior of a long cell of dimension greater than $k$. (Here one uses the fact that by definition the long cells are strictly longer than $\beta$.) Define $X(e)=\tilde{N}^{(k-1)} \cup\left\{\left|e^{\prime}\right| \mid e^{\prime} \in L^{\{k\}}, e^{\prime} \neq e\right\}$. As a first approximation to the foliated distance appearing in (i) we discuss the distance

$$
d(e, \alpha, \Delta)=d(Y(e, \alpha, \Delta), X(e)) .
$$

Observe that even though $X(e)$ is usually not compact only the intersection of it with some sufficiently large compact set matters. For small enough $\Delta$ we know that $Y(e, \alpha, \Delta) \neq \emptyset$ and hence $d(e, \alpha, \Delta)$ is a positive number, say $4 \epsilon_{e}^{\prime}$. Moreover $\Delta \leq \Delta^{\prime}$ implies $d(e, \alpha, \Delta) \leq d(e, \alpha, \Delta)$ and $d(e, \alpha, \Delta)$ tends to 0 if $\Delta$ does unless $e$ is a 0 -cell. For cells $e$ which are not 0 -cells and $0<\delta<\epsilon_{e}^{\prime}$ we define $\Delta_{e}(\alpha, \delta)$ as the minimal $\Delta$ for which $d(e, \alpha, \Delta) \geq 2 \delta$. Since each deck transformation $\gamma \in \Gamma$ acts by isometries, preserves the foliation, respects the filtration and permutes the cells, we know that $d(e, \alpha, \Delta)=d(\gamma e, \alpha, \Delta)$. Since there are only finitely many orbits of cells we can define $\epsilon^{\prime}$ as the minimal $\epsilon_{e}^{\prime}$, where $e$ ranges over all cells which are in $L^{\{k\}}$ for some $k \geq 0$, and define $\Delta(\alpha, \delta)$ as the maximal $\Delta_{e}(\alpha, \delta)$, where $e$ ranges over all cells which are in $L^{\{k\}}$ for some $k \geq 1$. For $0 \leq \alpha \leq \beta$ and $0<\delta<\epsilon_{L}^{\prime}$ and every $e \in L^{\{k\}}$ we have $d(e, \alpha, \Delta) \geq 2 \delta$ for all $\Delta \geq \Delta(\alpha, \delta)$ and $\Delta(\alpha, \delta)$ tends to 0 if $\delta$ does. It remains to improve the established inequalities slightly. Note first that for $\alpha \geq 0$ there is a constant $C_{f l w}(\alpha)$ such that for $x, y \in|\tilde{L}|$ with $d(x, y) \leq 1$ and all $t$ with $-\alpha \leq t \leq \alpha$ we have $d\left(\Phi_{t}(x), \Phi_{t}(y)\right)<C_{f l w}(\alpha) \cdot d(x, y)$ by a compactness argument. (For the geodesic flow on $S \mathbb{H} \tilde{M}$ this holds even over all of $S \mathbb{H} \tilde{M}$ by 4.3.) Because of our symmetric definition of foliated distance in Subsection 4.2 we see that $\Delta_{L}(\alpha, \delta)=\Delta\left(\alpha,\left(C_{f l w}(\alpha)+2\right) \cdot \frac{\delta}{2}\right)$ and $\epsilon_{L}^{\prime}=\min \left(1,\left(C_{f l w}(\beta)+1\right)^{-1} \cdot \frac{\epsilon^{\prime}}{2}\right)$ satisfy our requirements. Compare also Lemma 4.10](i). 
6.2. A family of flow cell structures. In order to prove foliated control results for $S \mathbb{H} \tilde{M}$ equipped with the geodesic foliation $F_{\text {geo }}$ we need longer and longer cell structures (necessarily missing more and more closed geodesics), but we also want to cover larger and larger chunks in the non-compact $\mathbb{H}$-direction (because the flow moves things in that direction). This naturally leads us to choose flow-cell structures $L_{\beta, i}$ indexed by $\mathbb{N}_{0} \times \mathbb{N}$ which are $\beta$-long and cover the $[-i, i]$-part in the $\mathbb{H}$-direction (each individual cell structure will only cover a compact region). Here are the details.

Let $\beta>0$ be given. Let $S \mathbb{H} M \leq \mu_{n} \beta$ denote the subset of $S \mathbb{H} M$ that consists of all closed geodesics of length $\leq \mu_{n} \beta$. Here $\mu_{n}=10^{n+3}$ with $n=\operatorname{dim} S \mathbb{H} M$; see Theorem 6.4. Observe that $S \mathbb{H} M \leq \mu_{n} \beta$ lies in $S \mathbb{H}_{\{0\}} M$ because all compact flow lines have an $\mathbb{H}$-coordinate which is constantly 0 .

For a fixed $\beta \in \mathbb{N}_{0}$ we choose a monotonic decreasing sequence of tubular neighborhoods $T_{\beta, i}, i \in \mathbb{N}$ of $S \mathbb{H} M \leq \mu_{n} \beta$ such that

$$
\bigcap_{i \in \mathbb{N}} T_{\beta, i}=S \mathbb{H} M \leq \mu_{n} \beta .
$$

We will use the tilde notation, i.e. $S \mathbb{H} \tilde{M} \leq \mu_{n} \beta$ and $\tilde{T}_{\beta, i}$, to denote the obvious preimages under the universal covering projection $S \mathbb{H} \tilde{M} \rightarrow S \mathbb{H} M$. Throughout the rest of this section we also fix a choice of a $\beta$-flow cell structure $L_{\beta, i}$ for $\beta \in \mathbb{N}_{0}$ and $i \in \mathbb{N}$ such that

$$
S \mathbb{H}_{[-i, i]} M-T_{\beta, i} \subset\left|L_{\beta, i}\right| \subset S \mathbb{H} M
$$

compare Theorem 6.4. Since its cells are shorter than $\beta \cdot \mu_{n} / 5$ we can also arrange that

$$
\left|L_{\beta, i}\right| \subset S \mathbb{H}_{\left[-i-\mu_{n} \beta, i+\mu_{n} \beta\right]} M .
$$

To our choice of flow cell structures $L_{\beta, i}$ we will now associate certain sequences of constants and functions. First recall that for a cell $e$ in a single flow cell structure $L$ we have the constants $\epsilon_{e}$ and $C_{e}$ appearing in Definition 6.1 and Remark 6.2 Moreover there are the constant $\epsilon_{L}^{\prime}$ and the function $\Delta_{L}(\alpha, \delta)$ from Proposition 6.5 We set

$$
\epsilon_{L}=\min \left\{\epsilon_{e} \mid e \in L\right\} \cup\left\{\epsilon_{L}^{\prime}\right\} \quad \text { and } \quad C_{L}=\max \left\{C_{e} \mid e \in L\right\} .
$$

Now go back to the family $\left(L_{\beta, i}\right)_{(\beta, i) \in \mathbb{N}_{0} \times \mathbb{N}}$ we have chosen above. We set

$$
\begin{aligned}
\epsilon_{i} & =\min \left\{\epsilon_{L_{\beta, i}} \mid 0 \leq \beta \leq i\right\}, \\
C_{i} & =\max \left\{C_{L_{\beta, i}} \mid 0 \leq \beta \leq i\right\}, \\
\Delta_{i}(\delta) & =\max \left\{\Delta_{L_{\beta, i}}(\alpha, \delta) \mid \alpha \in \mathbb{N}_{0} \text { and } 0 \leq \alpha \leq \beta \leq i\right\} \text { for } \delta<\epsilon_{i} .
\end{aligned}
$$

Note that $\Delta_{i}(\delta) \geq \delta$ and for $i$ fixed, $\Delta_{i}(\delta)$ tends to 0 with $\delta$; compare Proposition 6.5 (ii). For $\delta$ fixed, $\Delta_{i}(\delta)$ is monotonically increasing with $i$. Making the $\epsilon_{i}$ smaller and the $C_{i}$ larger if necessary we will assume that $\epsilon_{i}$ tends monotonically to 0 and the $C_{i}$ form an increasing sequence of numbers $>1$.

Later on, we will be in a situation where we can ignore all $L_{\beta, i}$ with $\beta>i$. (Compare Proposition 6.18 and the definition of $\mathcal{F}_{\mathbb{T}(\beta)}$ before that proposition.) With the above definitions the constants and functions labeled with $i$ have the desired properties simultaneously for all cell structures $L_{\beta, i}$ with $\beta \leq i$.

More precisely we have the following lemma. 


\section{Lemma 6.11.}

(i) For fixed $i$, Remark 6.3 with $\epsilon_{i}$ and $C_{i}$ instead of $\epsilon_{e}$ and $C_{e}$ applies simultaneously to all cells in all the cell structures $L_{\beta, i}$ with $\beta \leq i$.

(ii) Similarly for a fixed $\alpha \in \mathbb{N}_{0}$, Proposition 6.5 (i) applies with $\epsilon_{i}$ instead of $\epsilon_{L}^{\prime}$ to all cell structures $L_{\beta, i}$ with $\alpha \leq \beta \leq i$.

6.3. Construction of the decay speed $\mathcal{S}$. Let $\mathbf{t}=\left(t_{1}, t_{2}, \ldots\right)$ be a sequence of numbers with $t_{1}=1$ and $t_{i}<t_{i+1}$. Given a sequence $\left(\delta_{i}\right)$ we define the associated step-function $\operatorname{step}_{\mathbf{t}}\left(\left(\delta_{i}\right)\right)$ to be the function on $\mathbb{T}$ whose value on the interval $\left[t_{i}, t_{i+1}\right)$ is $\delta_{i}$. This defines a map from the space of sequences to the space of functions.

Our aim is now to construct a certain set of sequences $\mathcal{T}=\left\{\left(\delta_{i}\right)\right\}$ which will then (after a choice of a suitable sequence $\mathbf{t}$ ) lead to the set of functions $\mathcal{S}=\operatorname{step}_{\mathbf{t}}(\mathcal{T})$ used to describe the decay speed in the $\mathcal{E}_{w}$-control condition. In fact because of Remark 4.6] we are really only interested in the germs at infinity of such sequences.

Lemma 6.12. There exists a non-empty set $\mathcal{T}=\left\{\left(\delta_{i}\right)_{i \in \mathbb{N}}\right\}$ of sequences of positive numbers (each of which tends to zero) satisfying:

(i) Each sequence $\left(\delta_{i}\right) \in \mathcal{T}$ is eventually smaller than the sequence $\left(\epsilon_{i}\right)$ defined in equation (6.8); i.e., there exists an $i_{0} \in \mathbb{N}$ such that $\delta_{i}<\epsilon_{i}$ for all $i \geq i_{0}$.

(ii) For every $\left(\delta_{i}\right) \in \mathcal{T}$ the sequence $\left(\Delta_{i}\left(\delta_{i}\right)\right)$, eventually defined by (i), lies again in $\mathcal{T}$.

(iii) For every $\left(\delta_{i}\right) \in \mathcal{T}$ the sequence $\left(C_{i} \cdot \delta_{i}\right)$ lies again in $\mathcal{T}$.

Moreover we have the following more elementary properties corresponding to (A) and (B) before Definition 4.4.

(A) For $\left(\delta_{i}\right) \in \mathcal{T}$ and $k \in \mathbb{Z}$ we have $\left(\delta_{i+k}\right)_{i \in \mathbb{N}} \in \mathcal{T}$. (Here we set $\delta_{i+k}=\delta_{1}$ for $i+k \leq 0$.)

(B) Given $\left(\delta_{i}\right),\left(\delta_{i}^{\prime}\right) \in \mathcal{T}$ there exists $\left(\delta_{i}^{\prime \prime}\right) \in \mathcal{T}$ such that $\delta_{i}+\delta_{i}^{\prime} \leq \delta_{i}^{\prime \prime}$ for all $i \in \mathbb{N}$.

Proof. It will be convenient to define $\Delta_{i}^{\prime}(\delta)=\max \left\{2 \delta, \Delta_{i}(\delta), C_{i} \cdot \delta\right\}$ for $\delta \leq \epsilon_{i}$ and $\infty$ otherwise. For fixed $i$ this tends monotonically to 0 with $\delta$; for fixed $\delta$ it is monotonically increasing with $i$. The space $\mathcal{T}$ consisting of all $\left(\delta_{i}\right)$ satisfying the following condition

$$
\forall k, l \in \mathbb{Z}, j \in \mathbb{N}_{0} \quad \exists i_{0} \quad \forall i \geq i_{0} \quad\left(\Delta_{i+l}^{\prime}\right)^{\circ j}\left(\delta_{i+k}\right)<\epsilon_{i}
$$

is non-empty and satisfies (i), (ii), (iii), (A) and (B): Properties (i) and (A) are clear from this construction. To check property (ii) observe that

$$
\left(\Delta_{i+l}^{\prime}\right)^{\circ j}\left(\Delta_{i+k}\left(\delta_{i+k}\right)\right) \leq\left\{\begin{array}{lll}
\left(\Delta_{i+l}^{\prime}\right)^{\circ j+1}\left(\delta_{i+k}\right) & \text { if } & i+l \geq i+k, \\
\left(\Delta_{i+k}^{\prime}\right)^{\circ j+1}\left(\delta_{i+k}\right) & \text { if } & i+l \leq i+k .
\end{array}\right.
$$

Property (iii) follows by replacing $\Delta_{i+k}^{\prime}$ by $C_{i+k}$ in this inequality. To check (B) first observe that we have $\left(\Delta_{i}^{\prime}\right)^{\circ j}(2 \delta) \leq\left(\Delta_{i}^{\prime}\right)^{\circ j+1}(\delta)$. Thus, $\left(\delta_{i}\right) \in \mathcal{T}$ implies $\left(2 \delta_{i}\right) \in \mathcal{T}$. Moreover $\left(\delta_{i}\right) \in \mathcal{T}$ and $\delta_{i}^{\prime} \leq \delta_{i}$ implies $\delta_{i}^{\prime} \in \mathcal{T}$. Finally, by construction, $\left(\delta_{i}\right),\left(\delta_{i}^{\prime}\right) \in$ $\mathcal{T}$ implies $\max \left(\delta_{i}, \delta_{i}^{\prime}\right) \in \mathcal{T}$. So we get $(\mathrm{B})$ since $\delta_{i}+\delta_{i}^{\prime} \leq 2 \max \left(\delta_{i}, \delta_{i}^{\prime}\right)$. Since $\Delta_{i}^{\prime}(\delta)$ tends to 0 with $\delta$ we can find $\delta_{i}$ such that $\left(\Delta_{2 i}^{\prime}\right)^{\circ 2 i}\left(\delta_{i}\right)<\epsilon_{2 i}$. Then

$$
\left(\Delta_{i+l}^{\prime}\right)^{\circ j}\left(\delta_{i+k}\right) \leq\left(\Delta_{i+l}^{\prime}\right)^{\circ i+l}\left(\delta_{i+k}\right) \leq\left(\Delta_{2(i+k)}^{\prime}\right)^{\circ 2(i+k)}\left(\delta_{i+k}\right) \leq e_{2(i+k)}<\epsilon_{i}
$$

for sufficiently large $i$, i.e. if $i+l>j, 1 \leq i+l \leq 2(i+k)$ and $i \leq 2(i+k)$. Thus $\mathcal{T}$ contains $\left(\delta_{i}\right)$ and is indeed not empty. 
Now choose an increasing sequence $\mathbf{t}=\left(t_{1}, t_{2}, \ldots\right)$ with $t_{1}=1$ such that $t_{i+1}-$ $t_{i} \geq i$ (this will be important in Proposition [6.20) and $\left(e^{-a t_{i}}\right) \in \mathcal{T}$. Here $a$ is the curvature bound from (4.1). The next statement is immediate from Lemma 6.12 and this choice of $\mathbf{t}$.

Proposition 6.13. The set $\mathcal{S}=\operatorname{step}_{\mathbf{t}} \mathcal{T}$ satisfies the standard properties (A) and (B) of a class of decay speed functions introduced before Definition 4.4, each $\delta_{t} \in \mathcal{S}$ tends to zero for $t \rightarrow \infty$ and moreover:

(i) For each $A \cdot e^{-a t} \in \mathcal{S}_{\text {geo }}$ there exists a $\delta_{t} \in \mathcal{S}$ with $A \cdot e^{-a t} \leq \delta_{t}$; i.e., we can "relax control" from $\mathcal{S}_{\text {geo }}$ to $\mathcal{S}$.

(ii) For a given $\delta_{t}=\operatorname{step}_{\mathbf{t}}\left(\delta_{i}\right) \in \mathcal{S}=\operatorname{step}_{\mathbf{t}} \mathcal{T}$ take $i_{0} \in \mathbb{N}$ as in Lemma 6.12 (i) and define for $t \geq t_{i_{0}}$ the function $\Delta_{t}=\operatorname{step}_{\mathbf{t}}\left(\Delta_{i}\left(\delta_{i}\right)\right)$. This function lies again in $\mathcal{S}$.

(iii) For a given $\delta_{t}=\operatorname{step}_{\mathbf{t}}\left(\delta_{i}\right) \in \mathcal{S}$ the function $C_{t} \cdot \delta_{t}=\operatorname{step}_{\mathbf{t}}\left(C_{i} \cdot \delta_{i}\right)$ lies again in $\mathcal{S}$.

Condition (i) is important to obtain the map (5) in our main diagram in Section 3 i.e. to connect the following constructions with the kind of control we obtained via the geodesic flow. The second and third condition will play an important role in Proposition 6.24 respectively in Lemma 6.25 below.

6.4. Statement of the Foliated Control Theorem. Now we are prepared to define the control structures $\mathcal{E}_{w}$ and $\mathcal{E}_{s}$ on $S \mathbb{H} \tilde{M} \times \mathbb{B} \times \mathbb{T}$ which were already mentioned in the outline of the proof in Section 3. Let us recall what happened so far. For all natural numbers $\beta \geq 0$ and $i \geq 1$ we chose a tubular neighborhood $T_{\beta, i}$ of $S \mathbb{H} \tilde{M}^{\leq \mu_{n} \beta}$, and $\beta$-flow cell structures $L_{\beta, i}$ such that $S \mathbb{H}_{[-i, i]} M-T_{\beta, i} \subset\left|L_{\beta, i}\right|$. The associated constants $C_{L_{\beta, i}}$, the $\epsilon_{L_{\beta, i}}$ (see (6.7), Remarks 6.2) and 6.3) and the functions $\Delta_{L_{\beta, i}}(\alpha, \delta)$ given by Proposition 6.5 were used to define the sequences $\epsilon_{i}$, $C_{i}$ and $\Delta_{i}(\delta)$. In Lemma 6.12 we produced a space of sequences $\mathcal{T}$ out of this data. Before Proposition 6.13 we then chose a sequence $\mathbf{t}=\left(t_{1}, t_{2}, \ldots\right)$ and defined the set of decay speed functions $\mathcal{S}=\operatorname{step}_{\mathbf{t}}(\mathcal{T})$.

Now set

$$
S=\bigcup_{(\beta, i) \in \mathbb{N}_{0} \times \mathbb{N}}\left|L_{\beta, i}\right| \times[\beta, \beta+1] \times\left[t_{i}, t_{i+1}\right] \subset S \mathbb{H} \tilde{M} \times \mathbb{B} \times \mathbb{T} .
$$

The Foliated Control Theorem 6.17 will improve control precisely over $S$. Equip $\mathbb{B}$ with the Euclidean metric and $S \mathbb{H} \tilde{M} \times \mathbb{B}$ with some product metric. Equip $\mathbb{B}$ with the 0 -dimensional foliation by points and let $F_{w}$ denote the product foliation with the foliation $F_{\text {geo }}$ on $S \mathbb{H} \tilde{M}$ given by the geodesic flow.

Definition 6.15 (Weak and strong control).

(i) The "weak" morphism control condition $\mathcal{E}_{w}$ on $S \mathbb{H} \tilde{M} \times \mathbb{B} \times \mathbb{T}$ is defined as foliated control with respect to the foliation $F_{w}$ on $S \mathbb{H} \tilde{M} \times \mathbb{B}$ with decay speed $\mathcal{S}=\operatorname{step}_{\mathbf{t}}(\mathcal{T})$, i.e. $\mathcal{E}_{w}=\mathcal{E}\left(S \mathbb{H} \tilde{M} \times \mathbb{B}, F_{w}, \mathcal{S}\right)$; see Definition 4.4

(ii) The "very strong" morphism control condition $\mathcal{E}_{\text {vs }}$ on $S \mathbb{H} \tilde{M} \times \mathbb{B} \times \mathbb{T}$ is defined as metric control with respect to the product metric on $S \mathbb{H} \tilde{M} \times \mathbb{B}$ with decay speed $\mathcal{S}=\operatorname{step}_{\mathbf{t}}(\mathcal{T})$, i.e. $\mathcal{E}_{v s}=\mathcal{E}(S \mathbb{H} \tilde{M} \times \mathbb{B}, \mathcal{S})$; see Definition 4.4 We let $\mathcal{E}_{v s}^{\prime}$ denote $\mathcal{E}_{v s}$-control over the subset $S \subset S \mathbb{H} \tilde{M} \times \mathbb{B} \times \mathbb{T}$. (This is a control condition over $S \mathbb{H} \tilde{M} \times \mathbb{B} \times \mathbb{T}$ as explained in Definition 8.12.) 
(iii) The "strong" morphism control condition $\mathcal{E}_{s}$ is defined as $\mathcal{E}_{w} \cap \mathcal{E}_{v s}^{\prime}$, i.e. foliated control everywhere and metric control over $S$.

Observe that strong and weak control differ only over the subset $S$, where $\mathcal{E}_{s}$ requires the stronger metric control instead of only foliated control.

Remark 6.16. For the foliation $F_{w}$ there is a foliated triangle inequality analogous to Lemma 4.10(i). Together with (A) and (B) (compare Proposition 6.13) it hence follows that $\mathcal{E}_{w}$ is closed under composition and indeed defines a morphism control condition; compare Warning 4.7.

Recall that in Section 3 we introduced the object support condition

$$
\mathcal{F}_{\mathbb{B}}=\left\{\left\{(v, \beta, t) \mid \beta \leq \beta_{0}\right\} \mid \beta_{0} \in \mathbb{B}\right\}
$$

on $S \mathbb{H} \tilde{M} \times \mathbb{B} \times \mathbb{T}$ and the subspace $(S \mathbb{H} \tilde{M} \times \mathbb{B} \times \mathbb{T}) \angle$ consisting of all $(v, \beta, t)$ with $|h(v)| \leq t+\mu_{n} \beta$. Here $h, \beta$ and $t$ denote the $\mathbb{H}$-, $\mathbb{B}$ - respectively $\mathbb{T}$-coordinates of a point $(v, \beta, t) \in S \mathbb{H} \tilde{M} \times \mathbb{B} \times \mathbb{T}$ and $\mu_{n}=10^{n+3}$ with $n=\operatorname{dim} S \mathbb{H} \tilde{M}$. After all these preparations we can finally formulate the main result of this section.

Theorem 6.17 (Foliated Control Theorem). The forget control map

$$
\mathcal{C}^{\Gamma}\left((S \mathbb{H} \tilde{M} \times \mathbb{B} \times \mathbb{T})_{\angle,} \mathcal{E}_{s}, \mathcal{F}_{\mathbb{B}}\right)^{\infty} \rightarrow \mathcal{C}^{\Gamma}\left((S \mathbb{H} \tilde{M} \times \mathbb{B} \times \mathbb{T})_{\angle}, \mathcal{E}_{w}, \mathcal{F}_{\mathbb{B}}\right)^{\infty}
$$

given by relaxing the $\mathcal{E}_{s}$-control condition to the $\mathcal{E}_{w}$-condition induces an equivalence in $K$-theory.

After two preliminary reduction steps this result will be proven by induction over the skeleta of a relative cell structure. The proof will occupy the rest of this section.

6.5. First reduction - delooping in the $\mathbb{B}$-direction. Suppose we are given an $\left(\alpha, \delta_{t}\right)$-controlled morphism. Then in a region of the space $S \mathbb{H} \tilde{M} \times \mathbb{B} \times \mathbb{T}$ where $\beta$ is larger than $\alpha$ and $t$ is very large the morphism is quite well adapted to the flow cells we will find there. Conversely we would like to ignore a certain region where we have no hope to prove a comparison result between foliated and metric control. To capture this idea we introduce further object support conditions. We define analogously to $\mathcal{F}_{\mathbb{B}}$ the following object support conditions on $S \mathbb{H} \tilde{M} \times \mathbb{B} \times \mathbb{T}$ :

$$
\begin{aligned}
\mathcal{F}_{\mathbb{T}} & =\left\{\left\{(v, \beta, t) \mid t \leq t_{0}\right\} \mid t_{0} \in \mathbb{T}\right\}, \\
\mathcal{F}_{\mathbb{T}(\beta)} & =\left\{\left\{(v, \beta, t) \mid t \leq t_{0}(\beta)\right\} \mid t_{0}: \mathbb{B} \rightarrow \mathbb{T} \text { a continuous function }\right\}, \\
\mathcal{F} & =\mathcal{F}_{\mathbb{B}} \cup \mathcal{F}_{\mathbb{T}(\beta)} .
\end{aligned}
$$

Observe that germs away from $\mathcal{F}_{\mathbb{T}}$ are the usual germs at infinity. Since the definition of $\mathcal{F}$ only involves the $\mathbb{B}$ - and the $\mathbb{T}$-coordinate we will later use the same notation for other (subsets of) spaces of the form $X \times \mathbb{B} \times \mathbb{T}$. We can reformulate the Foliated Control Theorem as follows.

Proposition 6.18. The map in Theorem 6.17 induces an equivalence in $K$-theory if and only if the map

$$
\mathcal{C}^{\Gamma}\left((S \mathbb{H} \tilde{M} \times \mathbb{B} \times \mathbb{T})_{\angle}, \mathcal{E}_{s}\right)^{>\mathcal{F}} \rightarrow \mathcal{C}^{\Gamma}\left((S \mathbb{H} \tilde{M} \times \mathbb{B} \times \mathbb{T})_{\angle}, \mathcal{E}_{w}\right)^{>\mathcal{F}}
$$

induces an equivalence in $K$-theory.

Observe that there is no longer a compactness condition in the $\mathbb{B}$-direction but instead of germs at infinity we now have germs away from $\mathcal{F}$. 
Proof. For the purpose of this proof we introduce the following abbreviation. For object support conditions $\mathcal{F}^{\prime}$ and $\mathcal{F}^{\prime \prime}$ and a morphism support condition $\mathcal{E}$ on $S \mathbb{H} \tilde{M} \times \mathbb{B} \times \mathbb{T}$ set

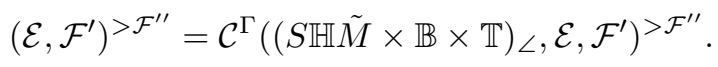

We have the following commutative diagram:

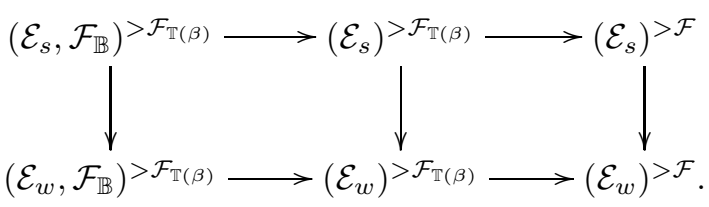

Here the vertical map on the left is the map in the Foliated Control Theorem 6.17 because under the presence of the $\mathcal{F}_{\mathbb{B}}$-object support condition there is no difference between germs away from $\mathcal{F}_{\mathbb{T}}$ (alias germs at infinity) and germs away from $\mathcal{F}_{\mathbb{T}(\beta)}$. According to Lemma 8.7 (iii) both rows yield fibration sequences in $K$-theory, and it hence suffices to show that both categories in the middle admit an Eilenberg swindle and are therefore contractible. Pick $\delta_{t} \in \mathcal{S}$. Then a swindle is induced in both cases by the map $(x, \beta, t) \mapsto\left(x, \beta+\delta_{t}, t\right)$ on $S \mathbb{H} \tilde{M} \times \mathbb{B} \times \mathbb{T}$; compare BFJR04. Proposition 4.4].

6.6. Second reduction - discretization. We would like to use the cell structures on the chunks $\left|L_{i, \beta}\right| \times[\beta, \beta+1] \times\left[t_{i}, t_{i+1}\right]$. But we do not know how around the boundary of the squares $[\beta, \beta+1] \times\left[t_{i}, t_{i+1}\right]$ the different cell structures fit together. To avoid this problem we use a Mayer-Vietoris argument.

We define the following subsets of $\mathbb{B}$ respectively $\mathbb{T}$ :

$$
\begin{aligned}
\mathbb{B}_{e}=\bigcup_{\substack{\beta \in \mathbb{N}_{0} \\
\beta \text { even }}}[\beta, \beta+1], & \mathbb{B}_{o}=\bigcup_{\substack{\beta \in \mathbb{N}_{0} \\
\beta \text { odd }}}[\beta, \beta+1], \\
\mathbb{T}_{e}=\bigcup_{\substack{i \in \mathbb{N} \\
i \text { even }}}\left[t_{i}, t_{i+1}\right], & \mathbb{T}_{o}=\bigcup_{\substack{i \in \mathbb{N} \\
i \text { odd }}}\left[t_{i}, t_{i+1}\right] .
\end{aligned}
$$

Moreover we set

$$
\mathbb{B}_{e \cap o}=\mathbb{B}_{e} \cap \mathbb{B}_{o}=\mathbb{N} \subset \mathbb{B}
$$

and

$$
\mathbb{T}_{\text {eกo }}=\mathbb{T}_{e} \cap \mathbb{T}_{o}=\left\{t_{1}, t_{2}, \ldots\right\} \subset \mathbb{T} .
$$

For a subspace $Y \subset S \mathbb{H} \tilde{M} \times \mathbb{B} \times \mathbb{T}$ we denote the intersection of $Y$ with $(S \mathbb{H} \tilde{M} \times$ $\mathbb{B} \times \mathbb{T})<$ by $Y_{\angle \text {. }}$

The condition that there are no non-trivial morphisms between different path components of $Y$ will be denoted by $\mathcal{E}_{\pi_{0}(Y)}$ or briefly $\mathcal{E}_{\pi_{0}}$. Formally this can be defined as the pull-back of the morphism control condition consisting only of the diagonal on $\pi_{0}(Y)$ via the natural projection $Y \rightarrow \pi_{0}(Y)$.

Warning 6.19. If $X$ is a subset of $Y$, one should not confuse $\mathcal{E}_{\pi_{0}(Y)}$ restricted to $X$, which is again denoted $\mathcal{E}_{\pi_{0}(Y)}$, with $\mathcal{E}_{\pi_{0}(X)}$.

Proposition 6.20. If for all 9 spaces $S \mathbb{H} \tilde{M} \times \mathbb{B}_{p} \times \mathbb{T}_{q}$ with $p, q \in\{e, o, e \cap o\}$ the maps

$$
\mathcal{C}^{\Gamma}\left(\left(S \mathbb{H} \tilde{M} \times \mathbb{B}_{p} \times \mathbb{T}_{q}\right)_{\llcorner}, \mathcal{E}_{s} \cap \mathcal{E}_{\pi_{0}}\right)^{>\mathcal{F}} \rightarrow \mathcal{C}^{\Gamma}\left(\left(S \mathbb{H} \tilde{M} \times \mathbb{B}_{p} \times \mathbb{T}_{q}\right)_{\llcorner}, \mathcal{E}_{w} \cap \mathcal{E}_{\pi_{0}}\right)^{>\mathcal{F}}
$$

induce equivalences in $K$-theory, then so does the map in Proposition 6.18 . 
Proof. If one drops the extra $\mathcal{E}_{\pi_{0}}$-condition on both sides, this follows easily by applying a Mayer-Vietoris argument (compare Remark 8.10) in the $\mathbb{T}$ - and then again in the $\mathbb{B}$-direction. However, dropping the $\mathcal{E}_{\pi_{0}}$-condition does not change the categories: if the support of a morphism in $\mathcal{C}^{\Gamma}\left(\left(S \mathbb{H} \tilde{M} \times \mathbb{B}_{p} \times \mathbb{T}_{q}\right)_{\angle}, \mathcal{E}_{s}\right)$ or $\mathcal{C}^{\Gamma}\left(\left(S \mathbb{H} \tilde{M} \times \mathbb{B}_{p} \times \mathbb{T}_{q}\right)_{\llcorner}, \mathcal{E}_{w}\right)$ violates this condition, it does so only on a set in $\mathcal{F}$, and this can be ignored since we take germs away from $\mathcal{F}$. For the $\mathbb{T}$-direction this follows from the fact that in the definition of foliated and metric control we always require a bound in the $\mathbb{T}$-direction and the fact that the distance between the $t_{i}$ increases with $i$. For the $\mathbb{B}$-direction it follows from the $\delta_{t}$-control in this direction.

From now on we will restrict our attention to the space $S \mathbb{H} \tilde{M} \times \mathbb{B}_{e} \times \mathbb{T}_{e}$, all the other cases being completely analogous.

6.7. Induction over the skeleta. We next define a filtration for $Y=S \mathbb{H} \tilde{M} \times$ $\mathbb{B}_{e} \times \mathbb{T}_{e}$. Recall that

$$
S=\bigcup_{\beta, i}\left|L_{\beta, i}\right| \times[\beta, \beta+1] \times\left[t_{i}, t_{i+1}\right] \subset S \mathbb{H} \tilde{M} \times \mathbb{B} \times \mathbb{T}
$$

and set

$$
\begin{aligned}
Y^{(-1)} & =\overline{\left(S \mathbb{H} \tilde{M} \times \mathbb{B}_{e} \times \mathbb{T}_{e}\right)-S}, \\
Y^{(k)} & =Y^{(-1)} \cup \bigcup_{\beta, i \text { even }}\left|L_{\beta, i}^{(k)}\right| \times[\beta, \beta+1] \times\left[t_{i}, t_{i+1}\right] .
\end{aligned}
$$

For a subspace $X \subset Y$ and a morphism support condition $\mathcal{E}$ on $Y$ we define the object support condition

$$
X^{\mathcal{E}}=\left\{X^{E} \mid E \in \mathcal{E}\right\} .
$$

(Recall that $X^{E}=\{y \in Y \mid$ there exists an $x \in X$ with $(x, y) \in E\}$ denotes the $E$-thickening of $X$ in $Y$.)

Proposition 6.21 (Induction Step). For $k=0,1, \ldots, n=\operatorname{dim} S \mathbb{H} \tilde{M}$ the relax control map

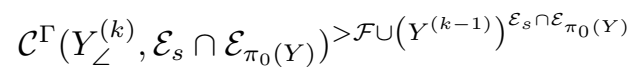

$$
\begin{aligned}
& \mathcal{C}^{\Gamma}\left(Y_{L}^{(k)}, \mathcal{E}_{w} \cap \mathcal{E}_{\pi_{0}(Y)}\right)^{>\mathcal{F} \cup\left(Y^{(k-1)}\right)^{\mathcal{E}_{w} \cap \mathcal{E}_{\pi_{0}(Y)}}}
\end{aligned}
$$

induces an equivalence in $K$-theory.

This proposition is proven by combining Proposition 6.24, Lemma 6.25 and Lemma 6.26 Before we proceed we note that Proposition 6.21 implies the Foliated Control Theorem 6.17

Corollary 6.22. The map

$$
\mathcal{C}^{\Gamma}\left(Y_{L}, \mathcal{E}_{s} \cap \mathcal{E}_{\pi_{0}(Y)}\right)^{>\mathcal{F}} \rightarrow \mathcal{C}^{\Gamma}\left(Y_{L}, \mathcal{E}_{w} \cap \mathcal{E}_{\pi_{0}(Y)}\right)^{>\mathcal{F}}
$$

induces an equivalence in $K$-theory. By Propositions 6.20 and 6.18 this implies the Foliated Control Theorem 6.17. 
Proof of the corollary. Let us abbreviate

$$
\begin{aligned}
& \mathcal{C}_{x}^{(k)}=\mathcal{C}^{\Gamma}\left(Y_{L}^{(k)}, \mathcal{E}_{x} \cap \mathcal{E}_{\pi_{0}(Y)}\right)^{>\mathcal{F}}, \\
& \mathcal{C}_{x}^{(k, k-1)}=\mathcal{C}^{\Gamma}\left(Y_{\llcorner}^{(k)}, \mathcal{E}_{x} \cap \mathcal{E}_{\pi_{0}(Y)}\right)>\mathcal{F} \cup\left(Y^{(k-1)}\right)^{\mathcal{E}_{x} \cap \mathcal{E}_{\pi_{0}(Y)}}
\end{aligned}
$$

for $x=w, s$. By definition of $\mathcal{E}_{s}$ and $\mathcal{E}_{w}$ we have $\mathcal{C}_{w}^{(-1)}=\mathcal{C}_{s}^{(-1)}$. By Lemma 8.7 (iii) and Remark 8.9 the sequence

$$
\mathcal{C}_{x}^{(k-1)} \longrightarrow \mathcal{C}_{x}^{(k)} \longrightarrow \mathcal{C}_{x}^{(k, k-1)}
$$

induces for $x=w$ or $x=s$ a fibration sequence in $K$-theory and relaxing control from $s$ to $w$ yields a map of fibration sequences. The result follows by induction using Proposition 6.21.

Observe that in Proposition 6.21 taking germs away from $Y^{(k-1)}$ means in particular that everything that is relevant happens over $S$, i.e. the region covered by the cell structures (compare (6.14)). Hence we can ignore the difference between strong and very strong control. (Formally this is an application of Lemma 8.11 from the Appendix.) Moreover we can drop the $\angle$-subscript because

$$
Y^{(k)}-Y_{L}^{(k)} \subset Y^{(-1)} \subset Y^{(k-1)} .
$$

We obtain the following lemma.

Lemma 6.23. The map in Proposition 6.21 is equal to the relax control map

$$
\begin{aligned}
& \mathcal{C}^{\Gamma}\left(Y^{(k)}, \mathcal{E}_{v s} \cap \mathcal{E}_{\pi_{0}(Y)}\right)>\mathcal{F} \cup\left(Y^{(k-1)}\right)^{\mathcal{E}_{v s} \cap \mathcal{E}_{\pi_{0}(Y)}} \\
& \downarrow
\end{aligned}
$$

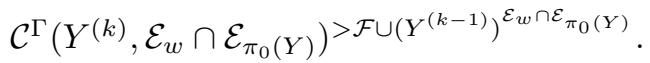

Recall that $L_{\beta, i}^{\{k\}}$ denotes those $k$-cells in $L_{\beta, i}$ which do not lie in the (-1)-st filtration step, i.e. in $Y^{(-1)}$. Set

$$
\begin{aligned}
Z^{(k)} & =\coprod_{\substack{e \in L_{\beta, i}^{\{k\}} \\
\beta, i \text { even }}}\left(A_{e} \times B_{e}\right) \times[\beta, \beta+1] \times\left[t_{i}, t_{i+1}\right], \\
\partial Z^{(k)} & =\coprod_{\substack{e \in L_{\beta, i}^{\{k\}} \\
\beta, i \text { even }}} \partial\left(A_{e} \times B_{e}\right) \times[\beta, \beta+1] \times\left[t_{i}, t_{i+1}\right] .
\end{aligned}
$$

There is a natural map

$$
g: Z^{(k)} \rightarrow Y^{(k)}
$$

induced by the charts $g_{e}$. The map induces a homeomorphism $\left(Z^{(k)}-\partial Z^{(k)}\right) \rightarrow$ $\left(Y^{(k)}-Y^{(k-1)}\right)$. Recall that the object support condition $\mathcal{F}$ is defined for every space with $\mathbb{B}$ - and $\mathbb{T}$-coordinate; compare the beginning of Subsection 6.5. In particular $g^{-1} \mathcal{F}$ will again be denoted $\mathcal{F}$. The following proposition is the crucial step in the proof of the Foliated Control Theorem and should be thought of as a "foliated excision" result. It allows separation of the cells. (Note the $\mathcal{E}_{\pi_{0}\left(Z^{(k)}\right)^{-}}$ condition in the source.) 
Proposition 6.24 (Excision of the $(k-1)$-skeleton). Let $\mathcal{E}$ denote either $\mathcal{E}_{v s}$ or $\mathcal{E}_{w}$. In both cases the natural map $g: Z^{(k)} \rightarrow Y^{(k)}$ induces an equivalence of categories

$$
\begin{aligned}
& \mathcal{C}^{\Gamma}\left(Z^{(k)}, g^{-1} \mathcal{E} \cap \mathcal{E}_{\pi_{0}\left(Z^{(k)}\right)}\right)>\mathcal{F} \cup\left(\partial Z^{(k)}\right)^{g^{-1} \mathcal{E} \cap \mathcal{E}} \pi_{0}\left(Z^{(k)}\right)
\end{aligned}
$$

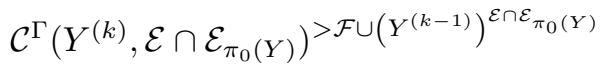

for $k=0,1, \ldots, n$.

Proof. Observe that the map factorizes over



Since $g: Z^{(k)} \rightarrow Y^{(k)}$ induces a homeomorphism $Z^{(k)}-\partial Z^{(k)} \rightarrow Y^{(k)}-Y^{(k-1)}$ and all conditions are simply pulled back along $g$, the second map in this factorization clearly induces an equivalence. We see that the crucial point is whether the forget control map from $g^{-1} \mathcal{E} \cap \mathcal{E}_{\pi_{0}\left(Z^{(k)}\right)}$-control to $g^{-1} \mathcal{E} \cap g^{-1} \mathcal{E}_{\pi_{0}(Y)^{-}}$-control induces an equivalence. Note that $\mathcal{E}_{\pi_{0}\left(Z^{(k)}\right)}$ does not allow morphisms between different cells and is hence a lot stronger than $g^{-1} \mathcal{E}_{\pi_{0}(Y)}$, which only separates the different $[\beta, \beta+$ $1] \times\left[t_{i}, t_{i+1}\right]$-blocks. Formally the result will be a consequence of Lemma 8.11 in the Appendix. We will apply that lemma to the case $X=Z^{(k)}, A=\partial Z^{(k)}$, $\mathcal{E}^{\prime}=g^{-1} \mathcal{E} \cap \mathcal{E}_{\pi_{0}\left(Z^{(k)}\right)}, \mathcal{E}^{\prime \prime}=g^{-1} \mathcal{E} \cap g^{-1} \mathcal{E}_{\pi_{0}(Y)}, \mathcal{F}_{0}=\emptyset$ and $\mathcal{F}=\mathcal{F}$. We only formulate the argument for $\mathcal{E}=\mathcal{E}_{w}$. The $\mathcal{E}_{v s^{-}}$-case is easier and can be obtained by setting $\alpha=0$; compare Remark 4.5.

Let $\alpha \in \mathbb{N}_{0}$ and $\delta_{t} \in \mathcal{S}$ be given. Write $E_{\alpha, \delta_{t}}$ for the subset $E \in \mathcal{E}$ determined as in Definition 4.4 (ii) by $\alpha$ and $\delta_{t}$ (we suppress the $t_{0}$ ). As in Proposition 6.13 (ii) define for the given $\delta_{t}=\operatorname{step}_{\mathbf{t}}\left(\left(\delta_{i}\right)\right) \in \mathcal{S}$ the function $\Delta_{t}=\operatorname{step}_{\mathbf{t}}\left(\left(\Delta_{i}\left(\delta_{i}\right)\right)\right)$ for $t \geq t_{i_{0}}$. In particular, $\delta_{i}<\epsilon_{i}$ for all $i \geq i_{0}$. By Proposition 6.13 (ii) we have $E_{\alpha, \Delta_{t}} \in \mathcal{E}$. Using our usual coordinates $\beta \in \mathbb{B}, t \in \mathbb{T}$ we define $F$ as the union of

$$
\begin{aligned}
F_{t \leq t_{i_{0}}} & =\left\{(v, \beta, t) \mid t \leq t_{i_{0}}\right\}, \\
F_{\beta \geq i} & =S \mathbb{H} \tilde{M} \times \bigcup_{\beta \geq i}[\beta, \beta+1] \times\left[t_{i}, t_{i+1}\right] \quad \text { and } \\
F_{\beta \leq \alpha} & =\{(v, \beta, t) \mid \beta \leq \alpha\} .
\end{aligned}
$$

Observe that $F_{t \leq t_{i_{0}}} \cup F_{\beta \geq i} \in \mathcal{F}_{\mathbb{T}(\beta)}, F_{\beta \leq \alpha} \in \mathcal{F}_{\mathbb{B}}$ and hence $F \in \mathcal{F}$. Let $E^{\prime \prime} \subset$ $g^{-1} E_{\alpha, \delta_{t}}$ resp. $E^{\prime} \subset g^{-1} E_{\alpha, \Delta_{t}}$ denote the subset of all pairs of points that satisfy in addition the $g^{-1} \mathcal{E}_{\pi_{0}(Y)^{-}}$-condition, resp. $\mathcal{E}_{\pi_{0}\left(Z^{(k)}\right) \text {-condition. We need to check }}$ that with this notation the condition in Lemma 8.11 is satisfied. Since $E_{\alpha, \delta_{t}} \subset$ $E_{\alpha, \Delta_{t}}$ it suffices to show that if $\left(x, x^{\prime}\right) \in E^{\prime \prime}$ and $x \notin\left(\partial Z^{(k)}\right)^{E^{\prime}} \cup F$, then $\left(x, x^{\prime}\right)$

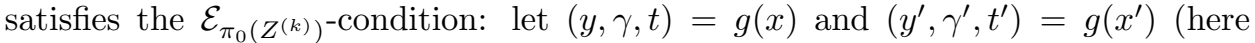
$\gamma$ is the $\mathbb{B}$ - and $t$ the $\mathbb{T}$-coordinate). There are $i, \beta$ such that $t_{i} \leq t, t^{\prime} \leq t_{i+1}$ and $\beta \leq \gamma, \gamma^{\prime} \leq \beta+1$, since $\left(x, x^{\prime}\right)$ satisfy the $\mathcal{E}_{\pi_{0}(Y)^{-c o n d i t i o n . ~ W e ~ k n o w ~} i \leq \beta}$ (since $x \notin F_{\beta \geq i}$ ), $\beta>\alpha$ (since $x \notin F_{\beta \leq \alpha}$ ) and $i>i_{0}$ (since $x \notin F_{t \leq t_{i_{0}}}$ ). In particular, $\delta_{i}<\epsilon_{i} \leq \epsilon_{L_{\beta, i}}$. There is $e \in L_{\beta, i}^{\{k\}}$ such that $y \in e-\partial e^{(\alpha, \Delta)}$ where $\Delta=\Delta_{i}\left(\delta_{i}\right) \geq \Delta_{L_{\beta, i}}\left(\alpha, \delta_{i}\right)$, since $x \notin\left(\partial Z^{(k)}\right)^{E^{\prime}}$. Finally, $d_{F_{\text {geo }}}\left(y, y^{\prime}\right) \leq\left(\alpha, \delta_{i}\right)$, since $\left(x, x^{\prime}\right)$ satisfies the $E_{\alpha, \delta_{t}}$-condition. All this allows the application of Lemma 6.5 (i) 
(compare also Lemma 6.11(ii) to conclude that $y^{\prime} \in e$. Thus $\left(x, x^{\prime}\right)$ does indeed satisfy the $\mathcal{E}_{\pi_{0}\left(Z^{(k)}\right)}$-condition.

6.8. Comparison to a Euclidean standard situation. According to the last proposition we can assume that all the cells which are new in the $k$-th step do not talk to each other through morphisms (this is formalized in the $\mathcal{E}_{\pi_{0}\left(Z^{(k)}\right)}$-condition). In the next step we will use the fact that each flow cell also has a security zone around it on which we have a very precise control over the foliation and the metric to prove that the situation is equivalent to a Euclidean standard situation. More precisely define

$$
W^{(k)}=\coprod_{\substack{e \in L_{\beta, i}^{\{k\}} \\ \beta, i \text { even }}}\left(\mathbb{R} \times \mathbb{R}^{n-1}\right) \times[\beta, \beta+1] \times\left[t_{i}, t_{i+1}\right] .
$$

We equip each $\left(\mathbb{R} \times \mathbb{R}^{n-1}\right) \times[\beta, \beta+1]$ with the standard Euclidean metric and with the foliation $F_{\mathbb{R}^{n}}$ (i.e. the foliation by lines parallel to the $\mathbb{R}$-factor). We define in the obvious way the foliated and the metric control structure on $W^{(k)}$. Namely in

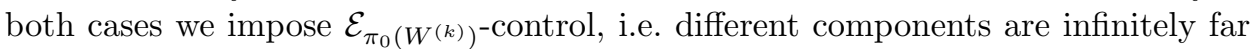
apart. We then let $\mathcal{E}_{m e t}$ denote metric control with decay speed $\mathcal{S}$ together with $\mathcal{E}_{\pi_{0}\left(W^{(k)}\right)}$ and $\mathcal{E}_{f o l}$ the foliated control with decay speed $\mathcal{S}$ together with $\mathcal{E}_{\pi_{0}\left(W^{(k)}\right)}$. We consider $Z^{(k)}$ as a subset of $W^{(k)}$ and denote this inclusion by $i: Z^{(k)} \rightarrow W^{(k)}$. Note that this map induces an equivalence on $\pi_{0}$ and hence $i^{-1} \mathcal{E}_{\pi_{0}\left(W^{(k)}\right)}=\mathcal{E}_{\pi_{0}\left(Z^{(k)}\right)}$. Now consider the following situation:

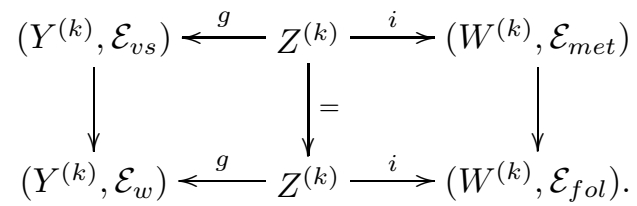

The following proposition reduces our problem to the Euclidean standard situation on the right of the diagram above.

Lemma 6.25 (Comparison to a Euclidean situation). For $k=0,1, \ldots, n$ the categories

$$
\mathcal{C}^{\Gamma}\left(Z^{(k)}, \mathcal{E}\right)^{>\mathcal{F} \cup\left(\partial Z^{(k)}\right)^{\mathcal{E}}}
$$

with $\mathcal{E}=g^{-1} \mathcal{E}_{v s} \cap \mathcal{E}_{\pi_{0}\left(Z^{(k)}\right)}$ resp. $\mathcal{E}=i^{-1} \mathcal{E}_{\text {met }}$ are equivalent. The same holds for the pair of control conditions $\mathcal{E}=g^{-1} \mathcal{E}_{w} \cap \mathcal{E}_{\pi_{0}\left(Z^{(k)}\right)}$ and $\mathcal{E}=i^{-1} \mathcal{E}_{\text {fol }}$.

Proof. We need to show that the four horizontal relax control maps in the following diagram are equivalences:

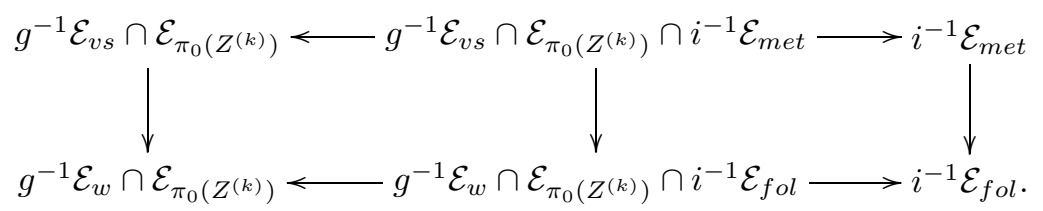

We only treat the lower left-hand horizontal map. The other cases are analogous. Formally the argument is an application of Lemma 8.11.

Let $\alpha$ and $\delta_{t} \in \mathcal{S}$ be given. Let $E^{\prime \prime}$ be determined by $g^{-1} E_{\alpha, \delta_{t}}$ and the $\mathcal{E}_{\pi_{0}\left(Z^{(k)}\right)^{-}}$ condition. Define $E^{\prime}$ by $i^{-1} E_{\alpha, C_{t} \delta_{t}} \cap E^{\prime \prime}$, where $C_{t}$ stems from Proposition 6.13 (iii) 
which also tells us that $C_{t} \delta_{t} \in \mathcal{S}$. Choose $i_{0}$ such that for all $i \geq i_{0}$ we have

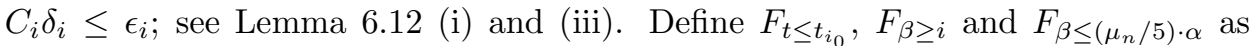
in the proof of Proposition 6.24 (but note the constant $\mu_{n} / 5$ ) and let $F$ be the union of these three sets. The condition in Lemma 8.11 is implied if one can show that two points in the same cell of a cell structure $L_{\beta, i}$, which are less than $\left(\alpha, \delta_{t_{i}}\right)$-foliated apart, when measured inside the manifold, are $\left(\alpha, C_{t_{i}} \delta_{t_{i}}\right)$-controlled, when measured in Euclidean space using the charts. At least this should be true away from the set $F$. But this is the content of Lemma 6.11)(i) which says that Remark 6.3 applies with $C_{i}$ instead of $C_{e}$ and with $\epsilon_{i}$ instead of $\epsilon_{e}$ if $\beta \leq i$. Note that we can assume $\alpha \leq 5 \mu_{n}^{-1} \beta$ and $t \geq t_{i_{0}}$, which translates into $\delta_{i} \leq C_{i}^{-1} \epsilon_{i}$. These are just the assumptions in Remark 6.3.

It remains to prove the comparison result for the Euclidean standard situation.

Lemma 6.26. For $k=0,1, \ldots, n$ the map

$$
\begin{gathered}
\mathcal{C}^{\Gamma}\left(Z^{(k)}, i^{-1} \mathcal{E}_{m e t}\right) \\
\mathcal{C}^{\Gamma}\left(Z^{(k)}, i^{-1} \mathcal{E}_{f o l}\right) \\
\downarrow
\end{gathered}
$$

induces an equivalence in $K$-theory.

Proof. Write $Z^{(k)}=Z_{t}^{(k)} \cup Z_{l}^{(k)}$ where the first subspace uses only transversal cells and the second only long cells. It suffices to check the claim separately for $Z_{t}^{(k)}$ and $Z_{l}^{(k)}$. On transversal cells $i^{-1} \mathcal{E}_{m e t}$ and $i^{-1} \mathcal{E}_{f o l}$ and the relevant thickenings of the boundaries agree and we are done for $Z_{t}^{(k)}$. If $A_{e}=[a, b]$ let $\partial_{-}^{e}=\{a\} \times B_{e}$ and write $\partial\left(A_{e} \times B_{e}\right)=\partial_{-}^{e} \cup \partial_{+}^{e}$ where $\partial_{+}^{e} \cap \partial_{-}^{e}=\{a\} \times \partial B_{e}$. Set

$$
\partial_{ \pm} Z_{t}^{(k)}=\coprod_{\substack{e \text { long cell in } L\{k\} \\ \beta, i \text { even }}} \partial_{ \pm}^{e} \times[\beta, \beta+1] \times\left[t_{i}, t_{i+1}\right] .
$$

Let $\mathcal{E}$ denote either $i^{-1} \mathcal{E}_{m e t}$ or $i^{-1} \mathcal{E}_{\text {fol }}$. Then according to Lemma 8.7)(iii) the $K$-theory of the categories we are interested in is the cofiber of the map induced by

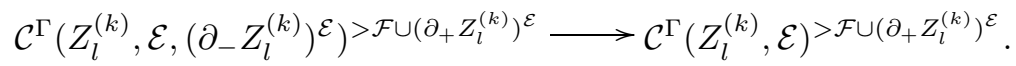

For both choices of $\mathcal{E}$ the map $(v, \gamma, t) \mapsto\left(v+\left(\delta_{t}, 0\right), \gamma, t\right)$ with some fixed $\delta_{t} \in \mathcal{S}$ induces an Eilenberg swindle for the category on the right. (Here $v=(a, b)$ refers to the coordinates in $A_{e} \times B_{e}$.) By Remark 8.9 the category on the left can be identified with

$$
\mathcal{C}^{\Gamma}\left(\partial_{-} Z_{l}^{(k)}, \mathcal{E}\right)^{>\mathcal{F} \cup\left(\partial_{+} Z_{l}^{(k)}\right)^{\mathcal{E}}} .
$$

Note that $\partial_{-} Z_{l}^{(k)}$ consists only of transversal pieces and we can repeat the argument from the beginning.

This finishes the proof of Proposition 6.21 and hence by Corollary 6.22 the proof of the Foliated Control Theorem 6.17 


\section{From strong CONTRol to COntinuous CONTROL}

In this section we explain the map (8) in the main diagram in Section 3 which connects the "strong" control $\left(\mathcal{E}_{s}\right.$-control) we obtained so far with the equivariant continuous control on the space $X(\infty) \times \mathbb{T}$ (denoted $\mathcal{E}_{\Gamma c c}(X(\infty))$-control).

7.1. A space with infinite cyclic isotropy. We recall the construction of the metric space $X$ and the map $p_{X}: S \mathbb{H} \tilde{M} \rightarrow X$ from [BFJR04 Section 14]. One can collapse $\mathbb{H} \tilde{M}$ to the $\Gamma$-compact space $\mathbb{H}_{[-1,1]} \tilde{M}$ by projecting $\mathbb{H}_{(-\infty,-1]} \tilde{M}$ in the obvious way to $\mathbb{H}_{\{-1\}} \tilde{M}$ and likewise $\mathbb{H}_{[1, \infty)} \tilde{M}$ to $\mathbb{H}_{\{1\}} \tilde{M}$. Similarly $X$ is obtained from $S \mathbb{H} \tilde{M}$, where additionally the fibers of the bundle $S \mathbb{H} \tilde{M} \rightarrow \mathbb{H} \tilde{M}$ over $\mathbb{H}_{\{-1\}} \tilde{M}$ and $\mathbb{H}_{\{1\}} \tilde{M}$ are collapsed to points. This collapsing map is the map $p_{X}: S \mathbb{H} \tilde{M} \rightarrow X$. More details of the construction can be found before Proposition 14.5 in [BFJR04]. We can also project all the way down to $\tilde{M}$ and we hence obtain a factorization of the natural projection $S \mathbb{H} \tilde{M} \rightarrow \tilde{M}$ over $X$. Restricted to $S \mathbb{H}_{[-0.5,0.5]} \tilde{M}$ the map $p_{X}$ is essentially the identity and we can hence consider $S \mathbb{H}_{[-0.5,0.5]} \tilde{M}$ and for every $\beta \geq 0$ also $S \mathbb{H} \tilde{M} \leq \mu_{n} \beta$ as a subset of $X$. (Recall that $S \mathbb{H} \tilde{M} \leq \mu_{n} \beta$ denotes the union of all leaves, which measured in $S \mathbb{H} M$ are shorter than $\mu_{n} \beta$.) Later the metric properties of $p_{X}$ will be important: $p_{X}$ does not increase distances and for $i$ large the map $p_{X}$ contracts $S \mathbb{H} \tilde{M}-S \mathbb{H}_{[-i, i]} \tilde{M}$ rather strongly. The precise statement is [BFJR04, 14.5].

We define $X(\beta)$ via the following push-out diagram whose horizontal arrows are $\Gamma$-cofibrations:

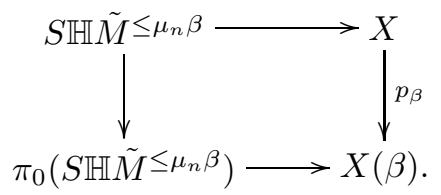

Since $S \mathbb{H} \tilde{M} \leq \mu_{n} \beta \subset S \mathbb{H} \tilde{M}^{\leq \mu_{n}(\beta+1)}$ we obtain natural maps $c_{\beta}: X(\beta) \rightarrow X(\beta+1)$ and we define $X(\infty)$ as the mapping telescope model for hocolim $\lim _{\beta \geq 0} X(\beta)$, i.e. as the coequalizer of

$$
\amalg_{\beta \geq 0} X(\beta) \Longrightarrow \coprod_{\beta \geq 0} X(\beta) \times[\beta, \beta+1],
$$

where the maps are given by sending $x \in X(\beta)$ to $(x, \beta+1)$ respectively to $\left(c_{\beta}(x), \beta\right)$. Note that $X(\infty)$ is a $\Gamma$-space all of whose isotropy groups are trivial or infinite cyclic. We obtain natural maps

$$
S \mathbb{H} \tilde{M} \times \mathbb{B} \stackrel{q}{\rightarrow} X \times \mathbb{B} \stackrel{p}{\rightarrow} X(\infty),
$$

where $q=p_{X} \times \operatorname{id}_{\mathbb{B}}$ and $p$ is induced from the maps $p_{\beta}$.

7.2. Strong control maps to continuous control. We will now check that $q$ does in fact define the arrow labeled (8) in the main diagram in Section 3 .

Proposition 7.1. The map $q$ induces a functor

$$
\mathcal{C}^{\Gamma}\left((S \mathbb{H} \tilde{M} \times \mathbb{B} \times \mathbb{T})_{\angle}, \mathcal{E}_{s}, \mathcal{F}_{\mathbb{B}}\right)^{\infty} \rightarrow \mathcal{C}^{\Gamma}\left(X \times \mathbb{B} \times \mathbb{T},\left(p \times \operatorname{id}_{\mathbb{T}}\right)^{-1} \mathcal{E}_{\Gamma c c}(X(\infty)), \mathcal{F}_{\mathbb{B}}\right)^{\infty}
$$

Proof. Note that the restriction of $q \times \mathrm{id}_{\mathbb{T}}$ to $(S \mathbb{H} \tilde{M} \times \mathbb{B} \times \mathbb{T}) \angle$ is a proper map. Compare the discussion of the map (4) in the outline of the proof in Section 3 We need to check that $q$ maps $\mathcal{E}_{s}$-control to $p^{-1} \mathcal{E}_{\Gamma c c^{-c o n t r o l}}$. This has roughly the following reasons: firstly, since $q$ does not increase distances ([BFJR04, 14.5(i)]) 
it maps very strong control $\left(\mathcal{E}_{v s}\right.$-control, compare Definition 6.15) to continuous control. Since $\mathcal{E}_{s}$-control implies $\mathcal{E}_{v s}$ over the set $S$ (which was defined at the beginning of Subsection [6.4), $\mathcal{E}_{s}$-control implies continuous control over $S$. Secondly, we need to deal with points on a geodesic $g$ that is collapsed to a point in $X(\infty)$. Here foliated control $\left(\mathcal{E}_{w}\right.$-control $)$ already implies continuous control. Thirdly, we are left with points that are not in $S$ because they have a large $\mathbb{H}-$ coordinate. Here $\mathcal{E}_{s}$-control implies only bounded control, but $q$ contracts this part very strongly ([BFJR04, 14.5(ii)]) and produces continuous control. For a careful argument one needs to construct suitable neighborhoods of $g$ that are invariant under the stabilizer of $g$ (compare [BFJR04 15.2]) and to use the fact that we did choose the tubular neighborhoods $T_{\beta, i}$ to be monotonically decreasing and such that $\bigcap_{i \in \mathbb{N}} T_{\beta, i}=S \mathbb{H} M \leq \mu_{n} \beta$.

\section{APPENDIX}

In this Appendix we collect a couple of facts which are more easily treated independently from the context in which they were used in the main text.

8.1. Homotopy finite chain complexes. Let $\overline{\mathcal{A}}$ be an additive category and $\mathcal{A} \subset \overline{\mathcal{A}}$ a full additive subcategory. We think of $\mathcal{A}$ as the category of "finite" objects in $\overline{\mathcal{A}}$; compare Subsection 5.2. Given such a situation we denote by $\operatorname{ch} \overline{\mathcal{A}}$ the category of bounded below chain complexes in $\overline{\mathcal{A}}$. The notion of chain homotopy leads to a notion of weak equivalence, and we define cofibrations to be those chain maps which are degreewise the inclusion of a direct summand. The category $\operatorname{ch} \overline{\mathcal{A}}$ becomes a Waldhausen category (a category with cofibrations and weak equivalences in the sense of [Wal85]). We define $\operatorname{ch}_{f} \mathcal{A}$ as the full subcategory of $\operatorname{ch} \overline{\mathcal{A}}$ whose objects are bounded below and above complexes where the object in each degree of the chain complex lies in $\mathcal{A}$. Furthermore we define $\operatorname{ch}_{h f} \mathcal{A}$ to be the full subcategory of chain complexes in $\operatorname{ch} \overline{\mathcal{A}}$ which are homotopy equivalent to a complex in $\operatorname{ch}_{f} \mathcal{A}$. The categories $\mathcal{A}, \operatorname{ch}_{f} \mathcal{A}$ and $\operatorname{ch}_{h f} \mathcal{A}$ inherit a Waldhausen structure from $\operatorname{ch} \overline{\mathcal{A}}$.

Lemma 8.1. The natural inclusions

$$
\mathcal{A} \rightarrow \operatorname{ch}_{f} \mathcal{A} \rightarrow \operatorname{ch}_{h f} \mathcal{A}
$$

induce equivalences in connective $K$-theory.

Proof. For the first map see [Bri79] and [TT90] or CP97]. The second inclusion induces an equivalence by a standard application of the Approximation Theorem 1.6.7 in Wal85]. (Mimic the mapping cylinder argument on page 380 in Wal85] for chain complexes.)

8.2. The tilde construction. In Section 5 we were forced to artificially enlarge the Waldhausen category $\operatorname{ch}_{h f} \mathcal{C} \Gamma(\tilde{E} \times \mathbb{T} ; \mathcal{E})$ to a category with the same $K$-theory in order to define a transfer. In fact this enlargement is most easily treated in the generality of Waldhausen categories. In this subsection we briefly describe how such an enlargement is formally defined and we explain that the natural inclusion defines an equivalence in $K$-theory under mild conditions.

Let $\mathcal{W}$ be a Waldhausen category. We additionally assume:

(M) Cofibrations in $\mathcal{W}$ are monomorphisms.

(H) There is a functor $\mathcal{C} \rightarrow \mathcal{D}$ to some other category such that precisely the weak equivalences are mapped to isomorphisms in $\mathcal{D}$. 
(Z) The category admits a cylinder functor and satisfies the cylinder axioms; compare page 348 in Wal85.

Note that $(\mathrm{H})$ implies the saturation axiom, i.e. the "two out of three"-axiom for weak equivalences (see Wal85. page 327).

Analogously to the Waldhausen categories $F_{m} \mathcal{W}$ defined on page 324 in Wal85] we define the Waldhausen category $F_{\infty} \mathcal{W}$ whose objects

$$
C=\left(C_{0} \stackrel{j_{0}}{\longrightarrow} C_{1} \stackrel{j_{1}}{\longrightarrow} C_{2} \stackrel{j_{2}}{\longrightarrow} \ldots\right)
$$

are infinite sequences of cofibrations in $\mathcal{W}$. We denote the full Waldhausen subcategory on those objects where all the $j_{i}$ are additionally weak equivalences by $\widehat{\mathcal{W}}$. The shift functor $s h: \widehat{\mathcal{W}} \rightarrow \widehat{\mathcal{W}}$ sends $C_{0} \rightarrow C_{1} \rightarrow \ldots$ to $C_{1} \rightarrow C_{2} \rightarrow \ldots$, i.e. it simply forgets $C_{0}$. There is an obvious natural transformation $\tau$ from the identity functor to the shift functor.

We define $\widetilde{\mathcal{W}}$ to be the category whose objects are the same as the objects in $\widehat{\mathcal{W}}$ and where the set of morphisms between $C$ and $D$ is given by the colimit over the following commutative diagram (which is indexed over the lattice points in a $\frac{3}{8}$-th plane):



Assumption (M) implies that all maps in this diagram are inclusions. We define the cofibrations in $\widetilde{\mathcal{W}}$ to be those morphisms which up to an isomorphism can be represented by a cofibration in $\widehat{\mathcal{W}}$ in the above colimit. Similarly $C \rightarrow D$ is a weak equivalence if it can be represented by a weak equivalence $s h^{n} C \rightarrow s h^{m} D$ in $\widehat{\mathcal{W}}$ for some $n$ and $m$. A lengthy but straightforward argument shows that these structures indeed define the structure of a Waldhausen category on $\widetilde{\mathcal{W}}$. Assumption $(\mathrm{H})$ is used to verify that isomorphisms are weak equivalences.

Proposition 8.2. Suppose $\mathcal{W}$ satisfies $(M),(H)$ and $(Z)$. Then the natural inclusion

$$
\mathcal{W} \rightarrow \widetilde{\mathcal{W}}
$$

which sends $C$ to $C \stackrel{\rightrightarrows}{\longrightarrow} C \stackrel{\rightrightarrows}{\longrightarrow} C \stackrel{\equiv}{\longrightarrow}$. induces an equivalence on connective K-theory.

Proof. This is an application of Waldhausen's Approximation Theorem 1.6.7 in Wal85.

8.3. Swan group actions on $K$-theory. In this subsection we will briefly describe several versions of the Swan group and how it acts on $K$-theory. We will use the notation

$$
S w(\Gamma ; \mathbb{Z}) \quad \text { and } \quad S w^{f r}(\Gamma ; \mathbb{Z})
$$

for the $K_{0}$-group of $\mathbb{Z} \Gamma$-modules which are finitely generated as $\mathbb{Z}$-modules respectively finitely generated free as $\mathbb{Z}$-modules. In both cases the relations are the 
additivity relation given for all (not necessarily $\mathbb{Z}$ - or $\mathbb{Z} \Gamma$-split) exact sequences. Furthermore we will need the "chain complex version"

$$
S w^{\mathrm{ch}}(\Gamma ; \mathbb{Z}),
$$

which is defined as the free abelian group on isomorphism classes of all bounded below complexes $C$ • of $\mathbb{Z} \Gamma$-modules satisfying

(i) the homology $H_{*}\left(C_{\bullet}\right)$ is finitely generated as an abelian group (and in particular concentrated in finitely many degrees);

(ii) the modules in each degree of $C \bullet$ are free as $\mathbb{Z}$-modules modulo the relations

(i) a short exact sequence $0 \rightarrow C_{\bullet} \rightarrow D_{\bullet} \rightarrow E_{\bullet} \rightarrow 0$ of $\mathbb{Z} \Gamma$-chain complexes yields $\left[D_{\bullet}\right]=\left[C_{\bullet}\right]+\left[E_{\bullet}\right]$;

(ii) if $C_{\bullet} \rightarrow D_{\bullet}$ is a $\mathbb{Z} \Gamma$-chain map which induces an isomorphism on homology, then $\left[C_{\bullet}\right]=\left[D_{\bullet}\right]$.

There is a natural map $i: S w^{f r}(\Gamma ; \mathbb{Z}) \rightarrow S w(\Gamma ; \mathbb{Z})$ and considering a module as a chain complex concentrated in degree 0 defines a map $j: S w^{f r}(\Gamma ; \mathbb{Z}) \rightarrow S w^{\mathrm{ch}}(\Gamma ; \mathbb{Z})$. More remarkable is the map

$$
\begin{aligned}
\chi: S w^{\mathrm{ch}}(\Gamma ; \mathbb{Z}) & \rightarrow S w(\Gamma ; \mathbb{Z}) \\
C_{\bullet} & \mapsto \sum(-1)^{i}\left[H_{i}\left(C_{\bullet}\right)\right] .
\end{aligned}
$$

Proposition 8.3. All three maps in the commutative diagram

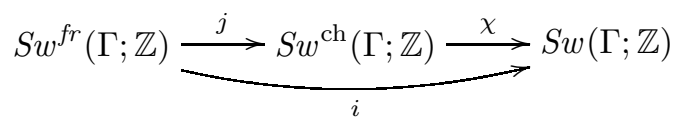

are isomorphisms.

Proof. For a $\mathbb{Z} \Gamma$-module $M$ we denote by $T M$ the $\mathbb{Z}$-torsion submodule. For $[M] \in$ $S w(\Gamma ; \mathbb{Z})$ there exists a $\mathbb{Z} \Gamma$-resolution $F_{\bullet}(T M)=\left(F_{1} \rightarrow F_{0}\right)$ of $T M$ by modules which are finitely generated free as $\mathbb{Z}$-modules. The map $\Phi:[M] \mapsto\left[F_{1}\right]-\left[F_{0}\right]+$ $[M / T M]$ is well defined and an inverse for $i$ by Lemma 2.2 in [PT78]. The claim now follows if we can prove that $j \circ \Phi \circ \chi$ is the identity. Let $\left[C_{\bullet}\right] \in S w^{\text {ch }}(\Gamma ; \mathbb{Z})$ be given. Without loss of generality we assume that the complex is concentrated in non-negative degrees. There is an $m \geq 0$ such that the degrees of all non-vanishing homology groups lie in $\{0,1, \ldots, m\}$. We argue by induction over $m$. Let $m=0$ and put $M=H_{0}\left(C_{\bullet}\right)$, i.e. $C_{\bullet}$ is a resolution of $M$. Choose projective $\mathbb{Z} \Gamma$-resolutions $P_{\bullet}^{\prime} \rightarrow T M$ and $P_{\bullet}^{\prime \prime} \rightarrow M / T M$. Then $P_{\bullet}=P_{\bullet}^{\prime} \oplus P_{\bullet}^{\prime \prime}$ is a resolution of $M$. Standard arguments produce chain maps $P_{\bullet}^{\prime} \rightarrow F_{\bullet}(T M), P_{\bullet}^{\prime \prime} \rightarrow M / T M$ and $P_{\bullet} \rightarrow C_{\bullet}$ which are homology isomorphisms. We have

$$
\begin{gathered}
j \circ \Phi \circ \chi\left(\left[C_{\bullet}\right]\right)=j \circ \Phi([M])=j\left(\left[F_{0}\right]-\left[F_{1}\right]+[M / T M]\right) \\
\quad=\left[F_{\bullet}(T M)\right]+[M / T M]=\left[P_{\bullet}^{\prime}\right]+\left[P_{\bullet}^{\prime \prime}\right]=\left[P_{\bullet}\right]=\left[C_{\bullet}\right] .
\end{gathered}
$$

Now let $m \geq 1$. Choose a projective $\mathbb{Z} \Gamma$-resolution of $L_{\bullet} \rightarrow H_{m}\left(C_{\bullet}\right)$ and construct an $H_{m}$-isomorphism $f: L_{\bullet} \rightarrow C_{\bullet}$. The map $f$ factorizes over its mapping cylinder and we have a short exact sequence

$$
0 \rightarrow L_{\bullet} \rightarrow \operatorname{cyl}(f) \rightarrow \operatorname{cone}(f) \rightarrow 0 .
$$

The claim holds by the induction hypothesis for $[\operatorname{cone}(f)]$ and by the argument for $m=0$ for $\left[L_{\bullet}\right]$ hence also for $\left[C_{\bullet}\right]=[\operatorname{cyl}(f)]$. 
Let $\mathcal{C}(\mathrm{pt} ; R \Gamma)$ denote the category of finitely generated free $R \Gamma$-modules. Each $\mathbb{Z} \Gamma$-module $M$ which is finitely generated free as a $\mathbb{Z}$-module yields an exact functor $-\otimes_{\mathbb{Z}} M: \mathcal{C}(\mathrm{pt} ; R \Gamma) \rightarrow \mathcal{C}(\mathrm{pt} ; R \Gamma)$. To check that this is well defined one uses that for a free $R \Gamma$-module $F$ there is a non-canonical isomorphism of $R \Gamma$-modules between $F \otimes_{\mathbb{Z}} M$ with the diagonal respectively with the left $\Gamma$-action. Using Proposition 1.3.1 and 1.3.2 (4) in Wal85] it is straightforward to check that the construction leads to maps

$$
S w^{f r}(\Gamma ; \mathbb{Z}) \otimes_{\mathbb{Z}} K_{n}(R \Gamma) \rightarrow K_{n}(R \Gamma)
$$

for $n \geq 0$. Replacing the one-point space pt by $\mathbb{R}^{n}$ (compare Remark 5.3) one obtains the corresponding construction for all $n \in \mathbb{Z}$. We use the isomorphism $i: S w^{f r}(\Gamma ; \mathbb{Z}) \rightarrow S w(\Gamma ; \mathbb{Z})$ to define maps

$$
S w(\Gamma ; \mathbb{Z}) \otimes_{\mathbb{Z}} K_{n}(R \Gamma) \rightarrow K_{n}(R \Gamma) .
$$

Remark 8.5. The tensor product over $\mathbb{Z}$ yields a ring structure on $S w^{f r}(\Gamma ; \mathbb{Z})$ and hence on $S w(\Gamma ; \mathbb{Z})$ and $S w^{\text {ch }}(\Gamma ; \mathbb{Z})$. The map (8.4) gives $K_{n}(R \Gamma)$ the structure of an $S w^{f r}(\Gamma ; \mathbb{Z})$-module.

Let $\operatorname{ch}_{h f} \mathcal{C}(\mathrm{pt} ; R \Gamma)$ denote the category of those bounded below chain complexes which are $\mathbb{Z} \Gamma$-homotopy equivalent to a bounded complex of finitely generated free $\mathbb{Z} \Gamma$-modules; compare Subsection 8.1. Let $C$. be a complex which represents an element in $S w^{\text {ch }}(\Gamma ; \mathbb{Z})$.

Lemma 8.6. The functor $-\otimes_{\mathbb{Z}} C_{\bullet}: \mathcal{C}(\mathrm{pt} ; R \Gamma) \rightarrow \operatorname{ch}_{h f} \mathcal{C}(\mathrm{pt} ; R \Gamma)$ is well defined.

Proof. Let $Q$ be a finitely generated free $\mathbb{Z} \Gamma$-module. Since each $C_{n}$ is free as a $\mathbb{Z}$-module there is a non-canonical isomorphism between $Q \otimes_{\mathbb{Z}} C_{n}$ equipped with the diagonal action and the same module with the right $\Gamma$-action. We see that $Q \otimes_{\mathbb{Z}} C_{\bullet}$ is a complex of free $\mathbb{Z} \Gamma$-modules. The crucial point is now to verify that this complex is homotopy equivalent to a bounded complex of finitely generated free $\mathbb{Z} \Gamma$-modules. We argue by induction over the length of the interval in which the homology of $C$ • is concentrated. We did this already in the proof of Proposition 8.3 and will use the notation established there. Let $m=0$ and let $C$ • be a $\mathbb{Z} \Gamma$ resolution of the module $M=H_{0}\left(C_{\bullet}\right)$. Since $Q$ is free as a $\mathbb{Z}$-module the sequence $0 \rightarrow Q \otimes_{\mathbb{Z}} T M \rightarrow Q \otimes_{\mathbb{Z}} M \rightarrow Q \otimes_{\mathbb{Z}} M / T M \rightarrow 0$ is exact. The diagonal-actionversus-right-action argument used above shows that $Q_{\mathbb{Z}} F_{\bullet}(T M)$ and $Q \otimes_{\mathbb{Z}} M / T M$ are finitely generated free $\mathbb{Z} \Gamma$-complexes. Using the horseshoe lemma we obtain a resolution $P_{\bullet}=\left(Q \otimes_{\mathbb{Z}} F_{1} \rightarrow Q \otimes_{\mathbb{Z}} F_{0} \oplus Q \otimes_{\mathbb{Z}} M / T M\right)$ of $M$ by a complex of finitely generated free $\mathbb{Z} \Gamma$-modules. Using standard arguments we can construct a $\mathbb{Z} \Gamma$ chain map $P_{\bullet} \rightarrow Q \otimes_{\mathbb{Z}} C_{\bullet}$ which is a homology isomorphism and hence a homotopy equivalence since both complexes are complexes of projective $\mathbb{Z} \Gamma$-modules. For the induction step one constructs a map $f: L_{\bullet} \rightarrow C_{\bullet}$ and a cylinder-cone sequence as in the proof of Proposition 8.3. One uses the induction hypothesis and the horseshoe lemma to construct a $\mathbb{Z} \Gamma$-homotopy equivalence $P_{\bullet} \rightarrow C_{\bullet}$ with $P_{\bullet}$ bounded and degreewise finitely generated $\mathbb{Z} \Gamma$-free.

Since the inclusion $\mathcal{C}(\mathrm{pt} ; R \Gamma) \rightarrow \operatorname{ch}_{h f} \mathcal{C}(\mathrm{pt} ; R \Gamma)$ induces an isomorphism in $K$ theory (compare Lemma 8.1) it is not difficult to check that we obtain maps

$$
S w^{\mathrm{ch}}(\Gamma ; \mathbb{Z}) \otimes_{\mathbb{Z}} K_{n}(R \Gamma) \rightarrow K_{n}(R \Gamma) .
$$


Via the isomorphism $j$ from Proposition 8.3 this action coincides with the action of $S w^{f r}(\Gamma ; \mathbb{Z})$ and $S w(\Gamma ; \mathbb{Z})$. In particular $-\otimes_{\mathbb{Z}} C_{\bullet}$ induces multiplication by $\sum(-1)^{i}\left[H_{i}\left(C_{\bullet}\right)\right]$.

8.4. Some fibration sequences. Let $\mathcal{E}$ be a morphism support condition on the $\Gamma$-space $X$ and let $\mathcal{F}, \mathcal{F}^{\prime}, \mathcal{F}_{0}$ and $\mathcal{F}_{1}$ be object support conditions. Slightly abusing set-theoretical notation we define $\mathcal{F}_{0} \cap \mathcal{F}_{1}=\left\{F_{0} \cap F_{1} \mid F_{0} \in \mathcal{F}_{0}, F_{1} \in \mathcal{F}_{1}\right\}$ and similarly $\mathcal{F}_{0} \cup \mathcal{F}_{1}$. Also we write $\mathcal{F}_{0} \subset \mathcal{F}_{1}$ if for every $F_{0} \in \mathcal{F}_{0}$ there is $F_{1} \in \mathcal{F}_{1}$ such that $F_{0} \subset F_{1}$. If $\mathcal{F}_{0} \subset \mathcal{F}_{1}$ and $\mathcal{F}_{1} \subset \mathcal{F}_{0}$ we write $\mathcal{F}_{0} \simeq \mathcal{F}_{1}$. We also use the corresponding notation for morphism support conditions.

An object support condition $\mathcal{F}$ is called $\mathcal{E}$-thickening closed if for every $F \in \mathcal{F}$ and $E \in \mathcal{E}$ there exists an $F^{\prime} \in \mathcal{F}$ such that $F^{E} \subset F^{\prime}$. Compare2.1.5 for notation. A typical example of such an $\mathcal{F}$ is $A^{\mathcal{E}}=\left\{A^{E} \mid E \in \mathcal{E}\right\}$ for a subset $A \subset X$. If $\mathcal{F}$ and $\mathcal{F}^{\prime}$ are $\mathcal{E}$-thickening closed object support conditions, then $\mathcal{C}^{\Gamma}\left(X, \mathcal{E}, \mathcal{F} \cap \mathcal{F}^{\prime}\right)$ is a Karoubi filtration $\operatorname{Kar70}$ of $\mathcal{C}^{\Gamma}(X, \mathcal{E}, \mathcal{F})$ and we define $\mathcal{C}^{\Gamma}(X, \mathcal{E}, \mathcal{F})^{>\mathcal{F}^{\prime}}$ as the Karoubi quotient. For the definition of Karoubi filtrations and quotients we refer to [CP97].

Lemma 8.7. For the purpose of this lemma we abbreviate

$$
\begin{aligned}
(\mathcal{F}) & =\mathcal{C}^{\Gamma}(X, \mathcal{E}, \mathcal{F}), \\
(\mathcal{F})^{>\mathcal{F}^{\prime}} & =\mathcal{C}^{\Gamma}(X, \mathcal{E}, \mathcal{F})^{>\mathcal{F}^{\prime}}
\end{aligned}
$$

and we assume that all object support conditions which occur are $\mathcal{E}$-thickening closed.

(i) The sequence $\left(\mathcal{F} \cap \mathcal{F}^{\prime}\right) \rightarrow(\mathcal{F}) \rightarrow(\mathcal{F})^{>\mathcal{F}^{\prime}}$ induces a fibration sequence in $K$-theory.

(ii) The square

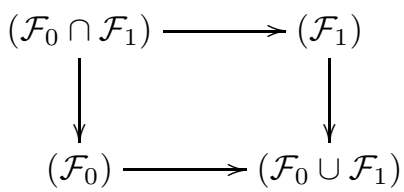

induces a homotopy push-out square of spectra after applying $K$-theory.

(iii) The sequence $\left(\mathcal{F} \cap \mathcal{F}_{0}\right)^{>\mathcal{F}_{1}} \rightarrow(\mathcal{F})^{>\mathcal{F}_{1}} \rightarrow(\mathcal{F})^{>\mathcal{F}_{0} \cup \mathcal{F}_{1}}$ induces a fibration sequence in $K$-theory.

Proof. The first statement is just the fact that a Karoubi filtration leads to a fibration sequence in $K$-theory; compare [CP97]. One can check that $\left(\mathcal{F}_{1}\right)^{>\mathcal{F}_{0} \cap \mathcal{F}_{1}} \rightarrow$ $\left(\mathcal{F}_{0} \cup \mathcal{F}_{1}\right)^{>\mathcal{F}_{0}}$ is an equivalence of categories. This yields (ii). The square

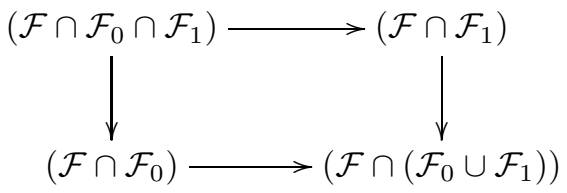

yields a homotopy push-out square by (ii). This square maps to the homotopy push-out square whose vertical maps are identities and both of whose horizontal maps are $\left(\mathcal{F} \cap \mathcal{F}_{0}\right) \rightarrow \mathcal{F}$. The induced square of cofibers is again a homotopy pushout square, and its lower left-hand corner is $\left(\mathcal{F} \cap \mathcal{F}_{0}\right)^{>\mathcal{F} \cap \mathcal{F}_{0}}$ and hence contractible. Using (i) this yields the desired fibration sequence in (iii). 
Example 8.8. Suppose that $\mathcal{E}$ and $\mathcal{F}$ are defined on $X \times[1, \infty)=X \times \mathbb{T}$ and let $\mathcal{F}_{\mathbb{T}}=\left\{X \times\left[1, t_{0}\right] \mid t_{0} \geq 1\right\}$. Then we write $\mathcal{C}^{\Gamma}(X \times \mathbb{T}, \mathcal{E}, \mathcal{F})^{\infty}$ for $\mathcal{C}^{\Gamma}(X \times \mathbb{T}, \mathcal{E}, \mathcal{F})^{>\mathcal{F}_{\mathbb{T}}}$ because we think of this category as being obtained by taking "germs at infinity". Let us assume that the next remark applies to the inclusion $X=X \times\{1\} \rightarrow X \times \mathbb{T}$. Then the fibration sequence from Lemma 8.7)(i) becomes

$$
\mathcal{C}^{\Gamma}(X, \mathcal{E}, \mathcal{F}) \rightarrow \mathcal{C}^{\Gamma}(X \times \mathbb{T}, \mathcal{E}, \mathcal{F}) \rightarrow \mathcal{C}^{\Gamma}(X \times \mathbb{T}, \mathcal{E}, \mathcal{F})^{\infty}
$$

and we refer to it as the "germs at infinity" fibration.

Remark 8.9. Let $i_{A}: A \rightarrow X$ be the inclusion of a $\Gamma$-invariant subset. Suppose that the morphism support condition $\mathcal{E}$ satisfies the following properness condition: for a compact $K \subset X$ and $E \in \mathcal{E}$ the closure of $K^{E}$ is again compact. Then

$$
\mathcal{C}^{\Gamma}\left(A, i_{A}^{-1} \mathcal{E}, i_{A}^{-1} \mathcal{F}_{0}\right)^{>i_{A}^{-1} \mathcal{F}} \rightarrow \mathcal{C}^{\Gamma}\left(X, \mathcal{E}, A^{\mathcal{E}} \cap \mathcal{F}_{0}\right)^{>\mathcal{F}}
$$

is an equivalence of categories. (Here $\mathcal{F}_{0}$ and $\mathcal{F}$ are again assumed to be $\mathcal{E}$ thickening closed object support conditions.) Most morphism support conditions used in this paper are defined on a locally compact metric space and contain a global metric condition and are therefore proper in the above sense. (In [BFJR04] a weaker properness condition was used, but this will not concern us here.)

Remark 8.10 (Mayer-Vietoris type results). Let $A$ and $B$ be $\Gamma$-invariant subsets of $X$ with $A \cup B=X$. Apply Lemma 8.7](ii) with $\mathcal{F}_{0}=A^{\mathcal{E}}$ and $\mathcal{F}_{1}=B^{\mathcal{E}}$. Suppose that $\mathcal{E}$ is proper in the sense of Remark 8.9 and that one can additionally show

$$
A^{\mathcal{E}} \cap B^{\mathcal{E}} \simeq(A \cap B)^{\mathcal{E}} \text {. }
$$

Then one obtains a Mayer-Vietoris result, i.e. a homotopy push-out square involving the $K$-theories of the four categories $\mathcal{C}^{\Gamma}\left(Y, i^{-1} \mathcal{E}\right)$, where $Y$ equals $A \cap B, A, B$ and $X$, respectively. (Here $i$ denotes in each case the relevant inclusion.) If $\mathcal{F}^{\prime}$ and $\mathcal{F}$ are $\mathcal{E}$-thickening closed object support conditions, then there is a similar homotopy push-out square for the categories $\mathcal{C}^{\Gamma}\left(Y, i^{-1} \mathcal{E}, i^{-1} \mathcal{F}\right)^{>i^{-1} \mathcal{F}^{\prime}}$.

Lemma 8.11. Let $X$ be a space and $A \subset X$ a subspace. Let $\mathcal{F}$ and $\mathcal{F}_{0}$ be object support conditions and $\mathcal{E}^{\prime} \subset \mathcal{E}^{\prime \prime}$ be morphism support conditions on $X$. The map

$$
\mathcal{C}\left(X, \mathcal{E}^{\prime}, \mathcal{F}_{0}\right)^{>\mathcal{F} \cup A^{\mathcal{E}^{\prime}}} \rightarrow \mathcal{C}\left(X, \mathcal{E}^{\prime \prime}, \mathcal{F}_{0}\right)^{>\mathcal{F} \cup A^{\mathcal{E}^{\prime \prime}}}
$$

induced by relaxing control from $\mathcal{E}^{\prime}$ to $\mathcal{E}^{\prime \prime}$ is an equivalence of categories provided the following condition is satisfied.

For all $E^{\prime \prime} \in \mathcal{E}^{\prime \prime}$ there exist $E^{\prime} \in \mathcal{E}^{\prime}$ and $F \in \mathcal{F} \cup A^{\mathcal{E}^{\prime}}$ such that

$$
E^{\prime \prime}-E^{\prime} \subset F \times F \text {. }
$$

Proof. Note that the condition implies $F \cup A^{\mathcal{E}^{\prime}}=F \cup A^{\mathcal{E}^{\prime \prime}}$. But it also implies that $\mathcal{C}\left(X, \mathcal{E}^{\prime}, \mathcal{F}_{0}\right)^{>\mathcal{F} \cup A^{\mathcal{E}^{\prime \prime}}} \rightarrow \mathcal{C}\left(X, \mathcal{E}^{\prime \prime}, \mathcal{F}_{0}\right)^{>\mathcal{F} \cup A^{\varepsilon^{\prime \prime}}}$ is surjective on objects and bijective on morphism sets.

The following concept was used to define strong control in Definition 6.15.

Definition 8.12 (Control over a subset). Let $\mathcal{E}$ be a control structure on the space $X$. Let $A \subset X$ be a subspace. We define $\mathcal{E}$-control over $A$ (a morphism-control condition on $X$ ) as follows. A morphism $\phi$ is $\mathcal{E}$-controlled over $A$ if there exists an $E \in \mathcal{E}$ such that if $(x, y)$ lies in the support of $\phi$ and $x$ or $y \in A^{-E}$, then $(x, y) \in E$.

Here $A^{-E}=\left(\left(A^{c}\right)^{E}\right)^{c}$ where $A^{c}$ denotes the complement of $A$ in $X$ and $A^{E}$ is the $E$-thickening of $A$ in $X$. 


\section{ACKNOWLEDGMENTS}

We would like to thank Tom Farrell and Lowell Jones for many discussions on the subject. We would also like to thank Wolfgang Lück for help with the Swan group actions. Our research was supported by the SFB 478 - Geometrische Strukturen in der Mathematik - Münster.

\section{REFERENCES}

[AS85] M. T. Anderson and R. Schoen, Positive harmonic functions on complete manifolds of negative curvature, Ann. of Math. (2) 121 (1985), no. 3, 429-461. MR0794369 (87a:58151)

[Bas68] H. Bass, Algebraic K-theory, W. A. Benjamin, Inc., New York-Amsterdam, 1968. MR.0249491 (40:2736)

[BBE85] W. Ballmann, M. Brin, and P. Eberlein, Structure of manifolds of nonpositive curvature. I, Ann. of Math. (2) 122 (1985), no. 1, 171-203. MR0799256 (87c:58092a)

[BCH94] P. Baum, A. Connes, and N. Higson, Classifying space for proper actions and $K$-theory of group $C^{*}$-algebras, $C^{*}$-algebras: 1943-1993 (San Antonio, TX, 1993), Contemp. Math., vol. 167, Amer. Math. Soc., Providence, RI, 1994, pp. 240-291. MR 1292018 (96c:46070)

[BFJR03] A. Bartels, F. T. Farrell, L. E. Jones, and H. Reich, A foliated squeezing theorem for geometric modules, High-dimensional manifold topology, World Sci. Publishing, River Edge, NJ, 2003, pp. 1-21. MR2048714(2004m:57046)

[BFJR04] - On the isomorphism conjecture in algebraic K-theory, Topology 43 (2004), no. 1, 157-213. MR2030590 (2004m:19004)

[BHS64] H. Bass, A. Heller, and R. G. Swan, The Whitehead group of a polynomial extension, Inst. Hautes Études Sci. Publ. Math. (1964), no. 22, 61-79. MR0174605 (30:4806)

[BO69] R. L. Bishop and B. O'Neill, Manifolds of negative curvature, Trans. Amer. Math. Soc. 145 (1969), 1-49. Mr 0251664 (40:4891)

[Bri79] K.-H. Brinkmann, Algebraische K-Theorie über Kettenkomplexe, Diplomarbeit, Bielefeld, 1979.

[CE75] J. Cheeger and D. G. Ebin, Comparison theorems in Riemannian geometry, NorthHolland Publishing Co., Amsterdam, 1975, North-Holland Mathematical Library, Vol. 9. MR0458335 (56:16538)

[CP97] M. Cárdenas and E. K. Pedersen, On the Karoubi filtration of a category, K-Theory 12 (1997), no. 2, 165-191. MR1469141 (98g:18012)

[DL98] J. F. Davis and W. Lück, Spaces over a category and assembly maps in isomorphism conjectures in $K$ - and L-theory, K-Theory 15 (1998), no. 3, 201-252. MR 1659969 (99m:55004)

[EHS93] P. Eberlein, U. Hamenstädt, and V. Schroeder, Manifolds of nonpositive curvature, Differential geometry: Riemannian geometry (Los Angeles, CA, 1990), Proc. Sympos. Pure Math., vol. 54, Amer. Math. Soc., Providence, RI, 1993, pp. 179-227. MR1216622 (94d:53060)

[EO73] P. Eberlein and B. O'Neill, Visibility manifolds, Pacific J. Math. 46 (1973), 45-109. MR0336648 (49:1421)

[FJ86] F. T. Farrell and L. E. Jones, K-theory and dynamics. I, Ann. of Math. (2) 124 (1986), no. 3, 531-569. MR0866708 (88f:57062)

[FJ87] , K-theory and dynamics. II, Ann. of Math. (2) 126 (1987), no. 3, 451-493. MR0916716 (89f:57029a)

[FJ89] — A topological analogue of Mostow's rigidity theorem, J. Amer. Math. Soc. 2 (1989), no. 2, 257-370. MR0973309 (90h:57023a)

[FJ91] Stable pseudoisotopy spaces of compact non-positively curved manifolds, J. Differential Geom. 34 (1991), no. 3, 769-834. MR1139646 (93b:57020)

[FJ93a] - Isomorphism conjectures in algebraic K-theory, J. Amer. Math. Soc. 6 (1993), no. 2, 249-297. MR.1179537(93h:57032)

[FJ93b] - Topological rigidity for compact non-positively curved manifolds, Differential geometry: Riemannian geometry (Los Angeles, CA, 1990), Proc. Sympos. Pure Math., vol. 54, Amer. Math. Soc., Providence, RI, 1993, pp. 229-274. MR1216623(94m:57067) 
[Gra76] D. Grayson, Higher algebraic K-theory. II (after Daniel Quillen), Algebraic K-theory (Proc. Conf., Northwestern Univ., Evanston, Ill., 1976), Springer, Berlin, 1976, pp. 217240. Lecture Notes in Math., Vol. 551. MR0574096 (58:28137)

[HIH77] E. Heintze and H.-C. Im Hof, Geometry of horospheres, J. Differential Geom. 12 (1977), no. 4, 481-491 (1978). MR0512919 (80a:53051)

[HP04] I. Hambleton and E. K. Pedersen, Identifying assembly maps in $K$ - and L-theory, Math. Ann. 328 (2004), no. 1-2, 27-57. MR2030369 (2004j:19001)

[HPR97] N. Higson, E. K. Pedersen, and J. Roe, $C^{*}$-algebras and controlled topology, $K$-Theory 11 (1997), no. 3, 209-239. MR1451755 (98g:19009)

[Hsi84] W. C. Hsiang, Geometric applications of algebraic K-theory, Proceedings of the International Congress of Mathematicians, Vols. 1, 2 (Warsaw, 1983) (Warsaw), PWN, 1984, pp. 99-118. MR0804679 (87g:57034)

[Kar70] M. Karoubi, Foncteurs dérivés et K-théorie, Séminaire Heidelberg-SaarbrückenStrasbourg sur la Kthéorie (1967/68), Lecture Notes in Mathematics, Vol. 136, Springer, Berlin, 1970, pp. 107-186. MR0265435 (42:344)

[Lod76] J.-L. Loday, $K$-théorie algébrique et représentations de groupes, Ann. Sci. École Norm. Sup. (4) 9 (1976), no. 3, 309-377. MR0447373 (56:5686)

[LR04] W. Lück and H. Reich, The Baum-Connes and the Farrell-Jones Conjectures in Kand L-Theory, arXiv:math.KT/0402405, to appear in Handbook of $K$-Theory, Eds. E. Friedlander, D. Grayson, Springer, 2004.

[Per01] L. Perko, Differential equations and dynamical systems, third ed., Texts in Applied Mathematics, vol. 7, Springer-Verlag, New York, 2001. MR1801796 (2001k:34001)

[PT78] E. K. Pedersen and L. R. Taylor, The Wall finiteness obstruction for a fibration, Amer. J. Math. 100 (1978), no. 4, 887-896. MR.0509078 (80g:55030)

[PW85] E. K. Pedersen and C. A. Weibel, A nonconnective delooping of algebraic K-theory, Algebraic and geometric topology (New Brunswick, N.J., 1983), Lecture Notes in Math., vol. 1126, Springer, Berlin, 1985, pp. 166-181. MR0802790 (87b:18012)

[PW89] $\quad$ K-theory homology of spaces, Algebraic topology (Arcata, CA, 1986), Lecture Notes in Math., vol. 1370, Springer, Berlin, 1989, pp. 346-361. MR.1000388 (90m:55007)

[Qui73] D. Quillen, Higher algebraic K-theory. I, Algebraic K-theory, I: Higher $K$-theories (Proc. Conf., Battelle Memorial Inst., Seattle, Wash., 1972), Springer, Berlin, 1973, pp. 85-147. Lecture Notes in Math., Vol. 341. MF0338129 (49:2895)

[Qui82] F. Quinn, Ends of maps. II, Invent. Math. 68 (1982), no. 3, 353-424. MR84j:57011

[tD72] T. tom Dieck, Orbittypen und äquivariante Homologie. I, Arch. Math. (Basel) 23 (1972), 307-317. MR0310919 (46:10017)

[tD87] , Transformation groups, de Gruyter Studies in Mathematics, vol. 8, Walter de Gruyter \& Co., Berlin, 1987. Mr0889050 (89c:57048)

[TT90] R. W. Thomason and T. Trobaugh, Higher algebraic K-theory of schemes and of derived categories, The Grothendieck Festschrift, Vol. III, Progr. Math., vol. 88, Birkhäuser Boston, Boston, MA, 1990, pp. 247-435. MR1106918 (92f:19001)

[Wal78] F. Waldhausen, Algebraic K-theory of generalized free products. I, II, Ann. of Math. (2) 108 (1978), no. 1, 135-204. MR0498807 (58:16845a)

[Wal85] Algebraic K-theory of spaces, Algebraic and geometric topology (New Brunswick, N.J., 1983), Lecture Notes in Math., vol. 1126, Springer, Berlin, 1985, pp. 318-419. MR0802796 (86m:18011)

Fachbereich Mathematik, Universität Münster, Einsteinstr. 62, 48149 Münster, GerMANY

E-mail address: bartelsa@math.uni-muenster.de

Fachbereich Mathematik, Universität Münster, Einsteinstr. 62, 48149 Münster, GerMANY

E-mail address: reichh@math.uni-muenster.de 